(W)

Check for

Cite as

Nano-Micro Lett.

(2022) 14:64

Received: 17 November 2021

Accepted: 28 December 2021

Published online: 23 February 2022

(C) The Author(s) 2022

\title{
A Better Zn-Ion Storage Device: Recent Progress for Zn-Ion Hybrid Supercapacitors
}

\author{
Jialun $\operatorname{Jin}^{1}$, Xiangshun Geng ${ }^{1}$, Qiang Chen $^{2}{ }^{凶}$, Tian-Ling Ren $^{1} \bowtie$
}

\section{HIGHLIGHTS}

- The advances of electrode materials, energy storage mechanisms, electrolytes and applications for Zn-ion hybrid supercapacitors (ZHSCs) are comprehensively summarized.

- Recent progresses in ZHSCs are discussed by categorizing into two configurations of Zn//Cap and Cap//ZBC.

- Future opportunities and challenges for the development of ZHSCs are also elaborated.

ABSTRACT As a new generation of Zn-ion storage systems, Zn-ion hybrid supercapacitors (ZHSCs) garner tremendous interests recently from researchers due to the perfect integration of batteries and supercapacitors. ZHSCs have excellent integration of high energy density and power density, which seamlessly bridges the gap between batteries and supercapacitors, becoming one of the most viable future options for large-scale equipment and portable electronic devices. However, the currently reported two configurations of ZHSCs and corresponding energy storage mechanisms still lack systematic analyses. Herein, this review will be prudently organized from the perspectives of design strategies, electrode configurations, energy storage mechanisms, recent advances in electrode materials, electrolyte behaviors and further applications (micro or flexible devices) of ZHSCs. The synthesis processes and electrochemical properties of well-designed $\mathrm{Zn}$ anodes, capacitor-type electrodes and novel Zn-ion battery-type cathodes are comprehensively discussed. Finally, a brief summary and outlook for the further development of ZHSCs are

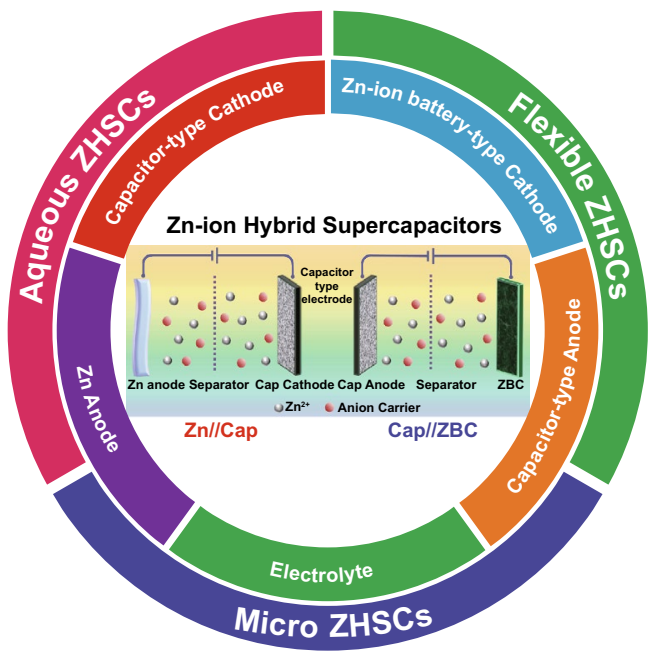
presented as well. This review will provide timely access for researchers to the recent works regarding ZHSCs.

KEYWORDS Zn-ion storage systems; Zn-ion hybrid supercapacitors; Carbon electrodes; Zinc anodes; Electrolytes

Qiang Chen, cq415@zjut.edu.cn; Tian-Ling Ren, rentl@tsinghua.edu.cn

1 School of Integrated Circuits and Beijing National Research Center for Information Science and Technology (BNRist), Tsinghua University,

Beijing 100084, People's Republic of China

2 College of Material Science and Engineering, Zhejiang University of Technology, Hangzhou 310014, People's Republic of China 


\section{Introduction}

As numerous portable electronic devices and electric vehicles are popularized and widely used, energy storage systems (ESSs) with excellent electrochemical performance (e.g., long cycling lifetime and high capacity) are playing a highly vital role in modern society $[1,2]$. Thus, various ESSs have been widely investigated and applied for commercial applications in recent years, such as metal-air batteries [3-5], aqueous batteries [6-10], supercapacitors [11-13] and others [14-22]. However, bottlenecks still exist in both batteries and supercapacitors, where the batteries have ultrahigh energy density but suffer from the short cycling lifetime due to excessive redox reactions while the supercapacitors possess predominant power density and long cycling lifetime but support fewer capacity than batteries [23-28]. Recently, to integrate respective advantages of batteries and supercapacitors into one individual device, the concept of hybrid supercapacitors (HSCs) become quite popular where the battery-type electrodes with abundant redox reaction act as an energy source and the capacitor-type (Cap) electrodes with fast ionic conductivity act as a power source [29-33]. Significantly, it is believed that the well-designed configuration of hybrid supercapacitors can bridge the gap between batteries and supercapacitors, and make the best use of both devices characteristics, showing an optimum ESSs for social demands [34-40].

Generally, metal-ion HSCs are diverse based on the different charge carriers like lithium-ion (Li-ion) [37, 41-43], sodium-ion (Na-ion) [44-49], potassium-ion (K-ion) [50-52] and zinc-ion (Zn-ion) [34, 53]. These HSCs have been widely studied and enormous developments have been made in recent years, such as Li-ion hybrid supercapacitors [54-57] and Na-ion hybrid supercapacitors [58-61]. Nevertheless, compared with the divalent $\mathrm{Zn}$-ion, alkaline metal-ions present some deficiencies in practical applications. For instance, the organic electrolytes employed in $\mathrm{Li}$ ion batteries (LIBs) and the excessively active redox reactions between alkaline metals and water/air, which lead to serious safety risks, potential environmental pollutions and complex manufacturing processes [35, 62-65]. Against this background, $\mathrm{Zn}$-ions show the smallest radius and highest electrode potential $(-0.76 \mathrm{~V}$ vs. standard hydrogen electrode, SHE) among the metal-ions mentioned above, enabling $\mathrm{Zn}$-ions to be highly efficient and safe charge carriers

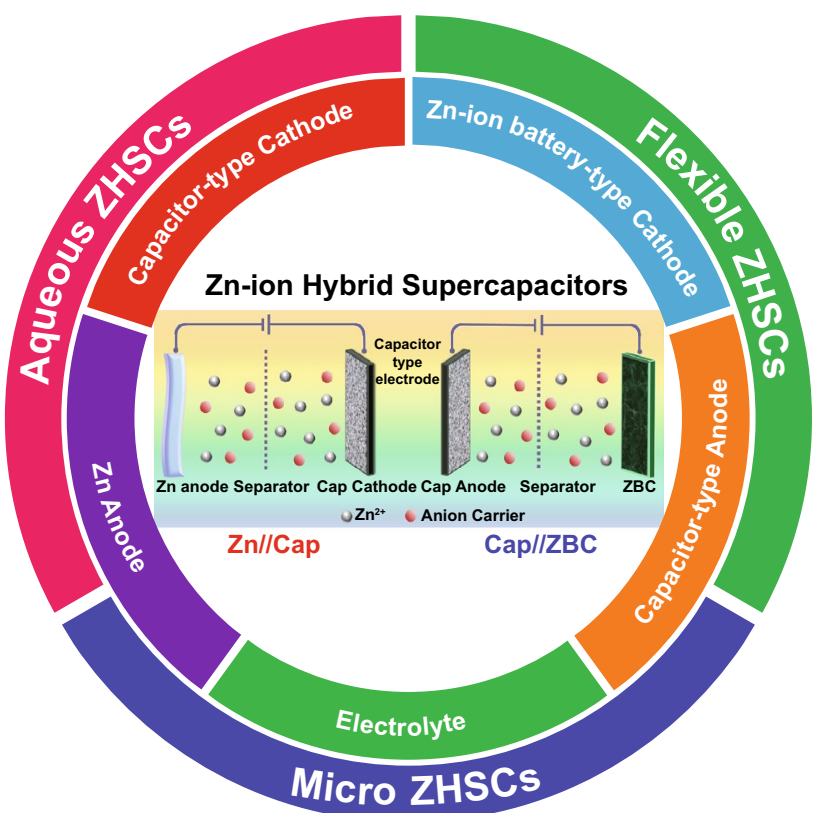

Scheme 1 Summary of the aspects discussed in this review and schematic illustration of two configurations ( $\mathrm{Zn} / / \mathrm{Cap}$ and Cap//ZBC) of ZHSCs

for aqueous HSCs [66, 67]. Notably, the Zn-ion ESSs have already been developed for almost 200 years, many Zn-ion ESSs showed the decisive impact on ESSs researches and have been intensively utilized in electronic devices, such as Zn-air [68-70], Zn-Ni [71-73], Zn-Ag [74-76] and various aqueous Zn-ion batteries (ZIBs) [67, 77]. Nowadays, wide applications of portable mobile electronic devices give rise to stricter requirements on the energy density and cycling ability of ESSs [78-81]. Hence, recently reported Zn-ion hybrid supercapacitors (ZHSCs) have exhibited attractive prospects include superb energy density, better power density, long cycling lifetime and splendid applications in flexible and micro-scale devices [32, 53, 82, 83]. Although some researches on ZHSCs have been developed, this novel ESS is still in its infancy, a systematic-and-comprehensive review including the design theories of two electrode configurations and energy storage mechanisms is necessary, which can be instructional for future investigations of ZHSCs [35, 84].

Herein, we systematically reviewed the recent progress in researches on ZHSCs. First, we classified the ZHSCs into two configurations according to the electrode materials and elaborated on the energy storage mechanisms of each configuration. As depicted in the Scheme 1, when the battery-type cathode or anode of ZIBs are substituted 
with Cap electrodes, the corresponding two configurations of ZHSCs are created. The first configuration of ZHSCs is constructed by the $\mathrm{Zn}$ anode and Cap cathode, while the second configuration of ZHSCs is assembled by Cap anode and $\mathrm{Zn}$-ion battery-type cathodes (ZBC). For brevity, these two configurations of ZHSCs are named as Zn//Cap ZHSCs and Cap//ZBC ZHSCs, respectively. In addition, the synthesis strategies, typical characteristics and electrochemical performance of electrode materials in each configuration of ZHSCs are introduced, including $\mathrm{Zn}$ anodes, various Cap electrodes and novel ZBC. Significantly, the formation mechanisms of by-products are carefully concluded and three typical energy storage mechanisms of Cap electrodes are summarized. The effects of various $\mathrm{Zn}$-based electrolytes and additive electrolytes on the $\mathrm{Zn}$ deposition and electrochemical performance of ZHSCs are emphasized. After that, we outlined the interesting application of ZHSCs for Zn-ion hybrid micro-supercapacitors (ZmSCs) and flexible ZHSCs devices. Finally, brief outlooks based on the recent ZHSCs progress are provided. We hope this review can attract more attention to this new generation $\mathrm{Zn}$-ion ESS and promote its further development and even practical applications in modern society.

\section{Aqueous Zn-Ion Hybrid Supercapacitors}

Due to the special electrode potential of $\mathrm{Zn}$ electrodes and suitable $\mathrm{Zn}$-ion salt electrolytes, the aqueous system of ZHSCs can be easily achieved and further promote the development of eco-friendly ESSs. In this section, we will summarize the recently reported aqueous ZHSCs system of two electrode configurations, the corresponding design theory, electrochemical performance and the energy storage mechanisms of electrode materials. Subsequently, some workable synthesis strategies of each electrode are introduced. Note that the Cap electrodes will be discussed between the two electrode configurations of ZHSCs.

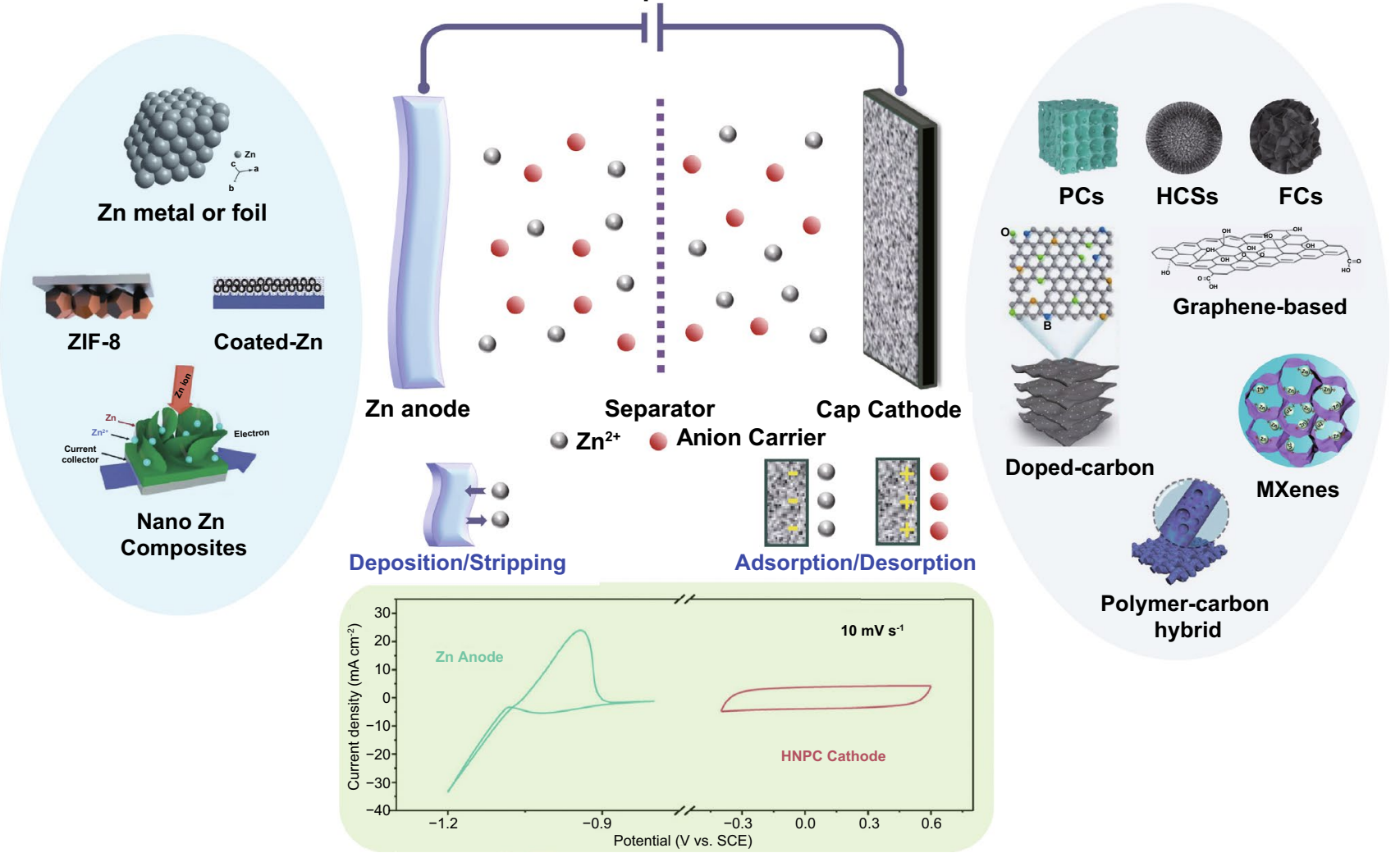

Fig. 1 Schematic illustration (Zn anodes, Cap cathodes, energy storage mechanisms and CV behaviors) of Zn//Cap ZHSCs 


\subsection{Zn//Cap ZHSCs}

The first electrode configuration of Zn//Cap ZHSCs is shown in Fig. 1, where Cap electrodes act as the cathode and power source, $\mathrm{Zn}$ foil or electrodeposited $\mathrm{Zn}$ composite electrodes act as the anode and energy source, and Zn salt solutions are employed as the electrolytes. To evaluate the energy storage mechanisms of Zn//Cap ZHSCs, the cyclic voltammetry (CV) curves of representative Cap cathode and $\mathrm{Zn}$ anode are depicted in Fig. 1 as well. The sharp redox peaks at $-1.2 \mathrm{~V} /-0.94 \mathrm{~V}$ (vs. saturated calomel electrode, SCE) are detected on the $\mathrm{Zn}$ anode, corresponding to the depositing/stripping processes of $\mathrm{Zn} / \mathrm{Zn}^{2+}$ redox reactions while the ideal rectangular shape is presented on the $\mathrm{N}$-doped hierarchically porous carbon (HNPC) cathode (represent most Cap electrodes) [85]. Typically, the rectangular CV curve indicated that the physical adsorption/desorption reaction is the dominant process on the surface of Cap electrodes, resulting in an electric double-layer capacitance (EDLC). Although the designed prototype of ZHSC is quite novel, many obstacles appeared when constructing high-performance ZHSCs due to the limitations of various electrode materials. Hence, we reviewed some reported $\mathrm{Zn}$ anodes in the system of $\mathrm{Zn} / /$ Cap ZHSCs and summarized feasible synthesis strategies for $\mathrm{Zn}$ anodes.

$\mathrm{Zn}$ anode, as the energy source of ZHSCs, proceeds with the depositing/stripping reaction of $\mathrm{Zn} / \mathrm{Zn}^{2+}$ during charging/discharging process. Generally, the depositing/stripping reaction equation can be expressed by Eq. (2-1) [34, 85]:

$\mathrm{Zn} \leftrightarrow \mathrm{Zn}^{2+}+2 \mathrm{e}^{-}$

$\mathrm{Zn}$ foil is a usual and suitable $\mathrm{Zn}$ anode material in the configuration of $\mathrm{Zn} / / \mathrm{Cap} \mathrm{ZHSCs}$ owing to the advantages of abundant redox reactions, low flammability, flexibility and low cost [86, 87]. Even so, similar to lithium dendrites in LIBs, Zn dendrite also presented on the surface of bare $\mathrm{Zn}$ foil, which can be a potential safety hazard for electronic devices [88, 89]. Generally, the formation mechanism of $\mathrm{Zn}$ dendrite can be ascribed to the uneven distribution of $\mathrm{Zn}$-ion contributed by the low-speed charging/discharging speed on the planar of two-dimensional (2D) Zn foil, which results in uneven nucleation and poor reversibility. The weeny tips on the pristine $\mathrm{Zn}$ foil can become the charge center of the electric field and further grow to $\mathrm{Zn}$ dendrites with the accumulation of charge. Although the formation of $\mathrm{Zn}$ dendrite can be suppressed by the fast charging/discharging property of well-designed ZHSCs, efforts should be devoted to thoroughly eliminating the Zn dendrite for the ESSs safety and promoting longevity of ZHSCs. Additionally, the hydrogen evolution induced by the $\mathrm{H}_{2} \mathrm{O}$ decomposition can greatly affect the depositing/stripping efficiency of flat $\mathrm{Zn}$ foil and increase the concentration of $\mathrm{OH}^{-}$in electrolyte, which may lead to the formation of by-products on the surface of both $\mathrm{Zn}$ anode and Cap cathode [90]. Therefore, a well-designed $\mathrm{Zn}$ anode with fast and high-efficient properties is necessary to realize high-performance ZHSCs. In the following section, we will summarize some strategies from the reported $\mathrm{Zn}$ anodes in ZHSCs: (1) Zn foil coating protection; (2) Electrodeposited nanostructure $\mathrm{Zn}$ anode; (3) Recoverable $\mathrm{Zn}$ anode.

\subsubsection{Zn Foil Coating Protection}

According to the above analysis of $\mathrm{Zn}$ dendrite, bare $\mathrm{Zn}$ foil with the planar and smooth surface is unfit for long cycling charging/discharging process. The $\mathrm{Zn}$ foil $/ / \mathrm{ZnSO}_{4}$ (gel) // layered B, N co-doped porous carbon (BN-LDC) ZHSCs reported by Lu et al. can reach a high capacity of $127.7 \mathrm{mAh}$ $\mathrm{g}^{-1}$ but only maintain capacity retention of $81.3 \%$ after 6500 cycles at $5 \mathrm{~A} \mathrm{~g}^{-1}$ [83]. Additionally, Sun et al. reported $\mathrm{Zn}$ foil// $/ \mathrm{Ti}_{3} \mathrm{C}_{2} \mathrm{ZHSCs}$ with a high capacity of $\sim 1 \mathrm{~F} \mathrm{~cm}^{-2}$ in the aqueous electrolyte of $\mathrm{ZnSO}_{4}$, however, the $\mathrm{Zn}$ foil//Ti ${ }_{3} \mathrm{C}_{2}$ ZHSCs presented an inferior cycling performance, where the retention decay to $78 \%$ of initial capacity after 4000 cycles at a higher current density of $10 \mathrm{~A} \mathrm{~g}^{-1}$ [91].

By far, many dendrite-free $\mathrm{Zn}$ anodes of ZHSCs have been reported and one of the most promising strategies is the $\mathrm{Zn}$ foil coating protection, which can greatly stabilize and improve the performance of $\mathrm{Zn}$ anode by covering stable components with a rough surface. As shown in Fig. 2a, Liu et al. applied mesoporous carbon hollow spheres (MCHSs) as the external protective layer of $\mathrm{Zn}$ foil anode, and simultaneously, the MCHSs are also employed as the cathode materials of ZHSCs [92]. The MCHS-coated Zn foil remained smooth and porous after long cycling test while some spikes emerged on the surface of the bare $\mathrm{Zn}$ foil. Typically, the external MCHSs protective layer can restrain and uniform the $\mathrm{Zn}$ dendrite during $\mathrm{Zn}$ depositing/stripping process. As a result, the MCHS-coated Zn foil//MCHSs can deliver excellent cycling performance of $96 \%$ capacity retention after 10,000 cycles at a low current density of $1 \mathrm{~A} \mathrm{~g}^{-1}$, indicating 
(a)

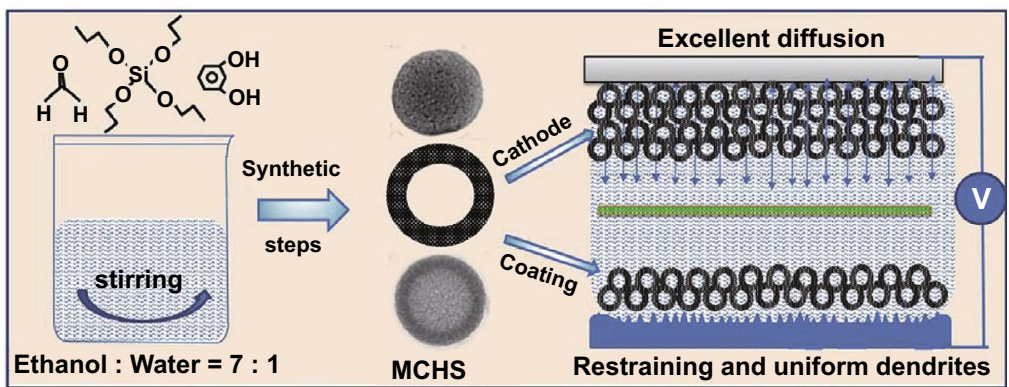

(c)
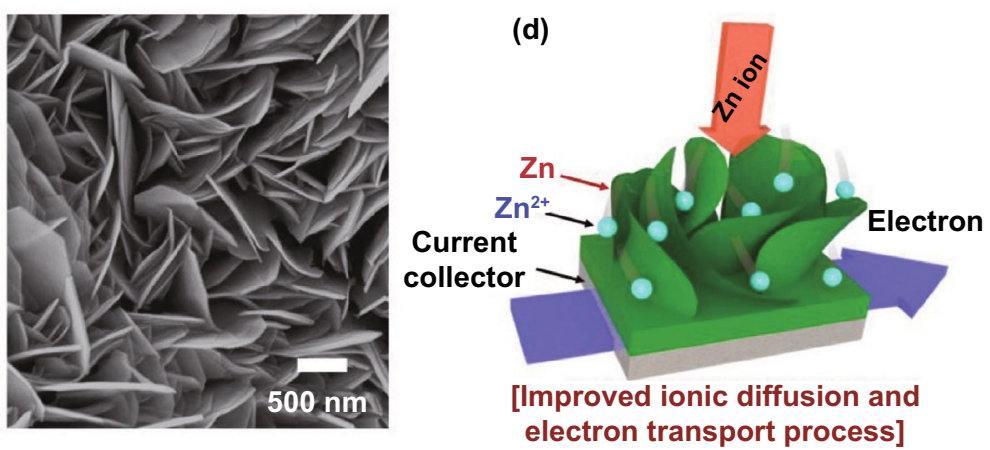
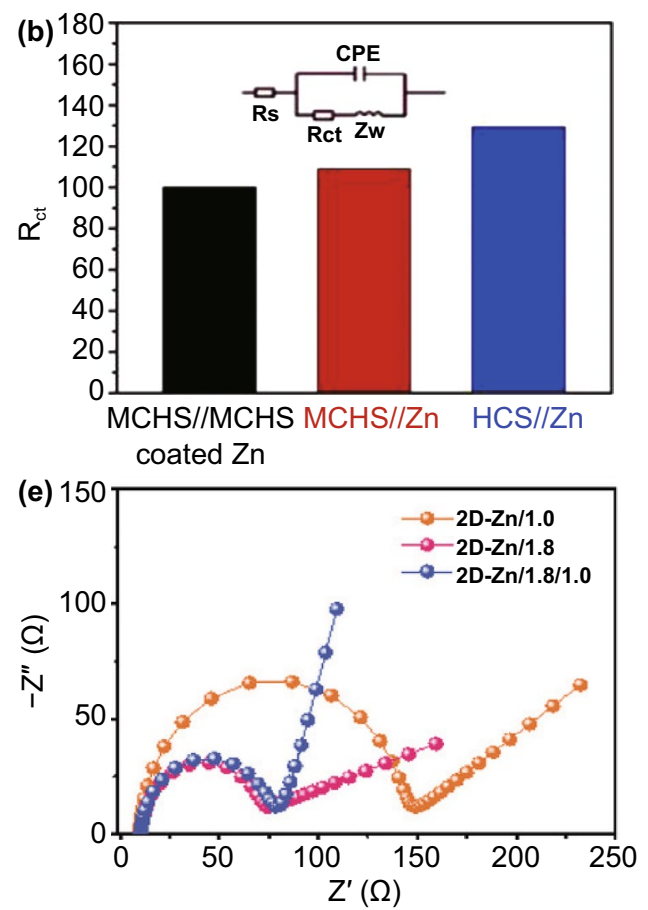

Fig. 2 a Schematic illustration of the synthesis process of MCHSs and corresponding application in electrodes of ZHSCs. b Comparison of $R_{\mathrm{ct}}$ value for the three devices. Reproduced with permission from Ref. [92]. Copyright 2019, Elsevier. c SEM image and d schematic illustration of the hierarchically 2D-Zn anode. e Nyquist plots of different 2D-Zn anodes. Reproduced with permission from Ref. [53]. Copyright 2020, Wiley$\mathrm{VCH}$

the good stability of MCHS-coated Zn foil. When tested at a battery-level current density of $0.1 \mathrm{~A} \mathrm{~g}^{-1}$, the MCHScoated $\mathrm{Zn}$ foil can circulate stably for 550 cycles, whereas the bare $\mathrm{Zn}$ foil suffered a large attenuation of capacity after only 350 cycles. It is believed that the coat-layer strategy can strongly stabilize the depositing/stripping reaction of $\mathrm{Zn}$ foil in the conditions of both fast and slow charging/ discharging process. Additionally, electrochemical impedance spectroscopy (EIS) measurement presented that the MCHS-coated $\mathrm{Zn}$ anodes have smaller charge transfer resistance $\left(R_{\mathrm{ct}}\right.$, Fig. $\left.2 \mathrm{~b}\right)$ and faster ion diffusion rate than bare $\mathrm{Zn}$ foil, verifying the high-efficient $\mathrm{Zn}$-ion depositing/stripping processes of MCHS-coated $\mathrm{Zn}$ anodes. Considering these results, the coat-layer strategy mentioned above may facilitate the splendid application of $\mathrm{Zn}$ foil in the ZHSCs field and then achieve better electrochemical performance.

\subsubsection{Electrodeposited Nanostructure Zn Anode}

By achieving a rough and relatively uniform surface, the electrodeposited $\mathrm{Zn}$ anode can greatly avoid the formation of
Zn dendrite and realize higher electrochemical performance [93]. In addition, electrodeposited $\mathrm{Zn}$ materials have been widely used as the $\mathrm{Zn}$ anodes in ZIBs before being applied in ZHSCs [32, 94]. However, the strategy is pretty different between the ZIBs and ZHSCs, where the electrodeposited $\mathrm{Zn}$ anode for ZHSCs requires not only high energy density but also high power density.

As shown in the scanning electron microscope image (SEM, Fig. 2c) and illustration (Fig. 2d), Cha et al. proposed a well-designed nanostructure of hierarchically electrodeposited $\mathrm{Zn}$ anode (2D-Zn) for ZHSCs, where the hierarchical structure of $\mathrm{Zn}$ is constructed with the close-knit background $\mathrm{Zn}$ and dispersive 2D nanostructure $\mathrm{Zn}$ on the surface [53]. Moreover, the electrode depositing strategy is carefully investigated by the three kinds of the electrodeposited 2D-Zn anode. The high voltage of $1.8 \mathrm{~V}$ electrodepositing process can uniformly cover the $\mathrm{Ni}$ form with close-knit background $\mathrm{Zn}$ while the low voltage of $1.0 \mathrm{~V}$ electrodepositing process can cover the Ni form with dispersive 2D nanostructure $\mathrm{Zn}$. Therefore, to achieve stable electron transport process and fast ionic diffusion rate simultaneously, the combined 
electrodepositing process of 1.8/1.0 V can manufacture a suitable hierarchical structure of electrodeposited $\mathrm{Zn}$ anode. Figure 2e depicts the EIS test results of three kinds of $2 \mathrm{D}-\mathrm{Zn}$ anode. The hierarchical structure of 2D-Zn/1.8/1.0 electrodes presented the superb electrochemical performance of low $R_{c t}$ and rapid ionic diffusion rate. Accordingly, two-step electrodepositing strategy can be a great method to synthesize the optimal nanostructure of $\mathrm{Zn}$ anode for ZHSCs, the as-assembled ZHSCs delivered an ultra-high energy density of $208 \mathrm{Wh} \mathrm{kg}^{-1}$ and a peak power density of $20,000 \mathrm{~W} \mathrm{~kg}^{-1}$. Meanwhile, remarkable cycling performance of $99 \%$ capacity retention after 10,000 cycles is achieved at $10 \mathrm{~A} \mathrm{~g}^{-1}$.
Since the formation of $\mathrm{Zn}$ dendrite can be effectively circumvented by utilizing electrodepositing strategy, the connection between electrodeposited $\mathrm{Zn}$ and substrate becomes very important, which directly relates to the electrochemical characteristics of $\mathrm{Zn}$ anode [95]. The substrate used for electrodepositing is a crucial part of achieving high-performance electrodeposited $\mathrm{Zn}$ anodes. Notably, the threedimensional (3D) printing (3DP) technique is employed by Sun et al. to form carbon nanotubes (CNTs) micro-lattice substrate for $\mathrm{Zn}$ electrodepositing [91]. Figure 3a displays the illustration of woodpile structure of the 3DP-CNT substrate, the unique structure based on the 3DP technique equipped the Zn@3DP-CNT with outstanding conductivity. (a)

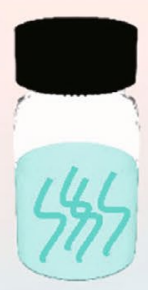

CNT ink

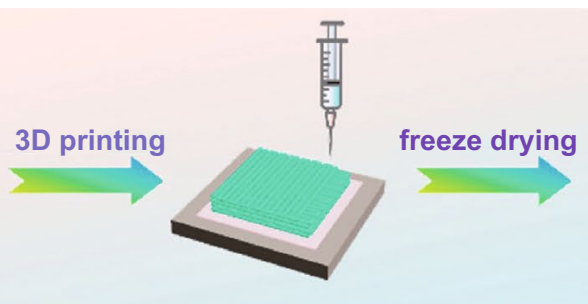

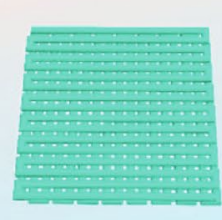

3DP CNT

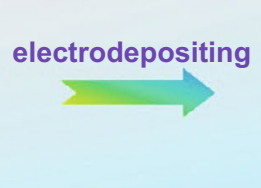

3DP CNT/Zn


Fig. 3 a Schematic illustration displaying the manufacturing process of 3DP-CNT/Zn anode. Reproduced with permission from Ref. [91]. Cop-

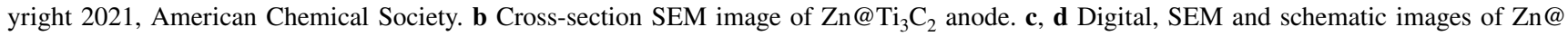
$\mathrm{Ti}_{3} \mathrm{C}_{2}$ anode after different time in PBS at $85^{\circ} \mathrm{C}$. Reproduced with permission Ref. [96] Copyright 2019, American Chemical Society. e Schematic illustration of the two-steps Zn plating on ZIF-8. Reproduced with permission from Ref. [90]. Copyright 2019, Elsevier. f Galvanostatic charge/discharge (GCD) curves of the devices after cycling and re-plating. Reproduced with permission from Ref. [97]. Copyright 2018, Royal Society of Chemistry 
Typically, the 3DP technique is a novel strategy for fabricating substrate for $\mathrm{Zn}$ anode, and the stereostructure of $\mathrm{Zn}$ anode may further promote the development of the electrodepositing method.

To implement the combination of high energy density and high power density, the electrodeposited $\mathrm{Zn}$ anodes are usually designed as high-surface-area structures and thin flakes. Thus, the as-designed structure of electrodeposited $\mathrm{Zn}$ anode may lead to a faster degradation rate than $\mathrm{Zn}$ foil due to the accelerated corrosion interaction. As depicted in Fig. 3b, Zhi et al. vertically electrodeposited the $\mathrm{Zn}$ nanoflakes onto the $\mathrm{Ti}_{3} \mathrm{C}_{2}$ film, the thickness of the $\mathrm{Zn}$ nanoflakes is $\sim 42 \mathrm{~nm}$ [96]. When the $\mathrm{Zn} @ \mathrm{Ti}_{3} \mathrm{C}_{2}$ electrode is soaked in the phosphate buffer saline solution (PBS, Fig. 3c, d) at $85^{\circ} \mathrm{C}$, part of $\mathrm{Zn}$ nanoflakes were dissolved into the PBS after 4 days, and finally eliminated as time prolonged to 7 days, which is much faster than the $\mathrm{Zn}$ foil (> 30 days). The degradable electrodepositing strategy makes the electrodeposited $\mathrm{Zn}$ anode and the ZHSCs more eco-friendly electrode and ESSs, respectively.

Porous structure of metal-organic framework (MOF) is also an excellent host for dendrite-free and fast diffusion $\mathrm{Zn}$-ion storage. Wang et al. prepared MOF Zn-zeolitic imidazolate framework (ZIF-8) electrodes with a 3D cage structure via optimized heat treatment $\left(500^{\circ} \mathrm{C}\right)$ [90]. After the heat treatment, the $\mathrm{Zn}^{2+}$ turned into the evenly distributed $\mathrm{Zn}^{0}$ in porous $\mathrm{ZIF}-8-500$, which can provide uniform nuclei for $\mathrm{Zn}$ depositing and high over-potential to reduce the $\mathrm{H}_{2} \mathrm{O}$ decomposition during the charging/discharging processes. As illustrated in Fig. 3e, the Zn electroplating process is divided into two steps, including the initial $\mathrm{Zn}$ plating and further $\mathrm{Zn}$ plating. Initial Zn plating normally proceeded in or on the ZIF-8-500 particle due to the uniform nuclei provide by the $\mathrm{Zn}^{0}$, and then further $\mathrm{Zn}$ plating occurred on the particle substrate and end with dendritefree Zn@ZIF-8-500 anode. Subsequently, Zn@ZIF-8-500 anode is coupled with activated carbon cathode to form ZHSCs and delivered an energy density of $140.8 \mathrm{Wh} \mathrm{kg}^{-1}$ as well as splendid longevity up to 20,000 cycles. Similarly, Shen et al. employed annealed ZIF-8 as the substrate of dendrite-free $\mathrm{Zn}$ anode and Zapien et al. deposited the $\mathrm{Zn}$ onto the flexible carbon cloth (CC) [98, 99]. The combination of electrodeposited $\mathrm{Zn}$ and carbon materials enables the composite $\mathrm{Zn}$ anode outstanding properties for highperformance ZHSCs.

\subsubsection{Recoverable Zn Anode}

In the high-performance ZHSCs devices, the irreversible consumption of the $\mathrm{Zn}$ anode is unavoidable and the endurance of the battery-type $\mathrm{Zn}$ anode is not satisfied with the long-term supercapacitors [100]. Therefore, a suitable strategy that can refresh the $\mathrm{Zn}$ electrodes is necessary. Sun et al. reported recoverable $\mathrm{Zn}$ anodes for ZHSCs by applying an in-situ electroplating method [97]. After re-plating process, no clear morphology alteration is detected and the re-plating $\mathrm{Zn}$ nanosheets presented vertical orientation, which may provide a lower resistance pathway for electron transfer. As presented in Fig. 3f, the re-plating $\mathrm{Zn}$ nanosheets anodes exhibited a higher capacity $\left(76 \mathrm{mF} \mathrm{cm}^{-2}\right)$ than the after-cycling (before re-plating) $\mathrm{Zn}$ nanosheets anodes $\left(60.9 \mathrm{mF} \mathrm{cm}^{-2}\right)$. Furthermore, the re-plating $\mathrm{Zn}$ nanosheets anodes maintained the capacity for additional 1500 cycles. Therefore, this simple operation can efficiently retard the irreversible recession of $\mathrm{Zn}$ anode during charging/discharging processes. Notably, the re-plating routine was replenished with in-situ method, thus the device structure will stay intact. Therefore, electronic devices can be easily refreshed through specific charging processes, and the recycling projects of ZHSCs can expediently renew these devices and apply them to other low-demand electronic devices.

Considering the above three strategies for $\mathrm{Zn}$ anodes in ZHSCs, it is believed that the hinges of $\mathrm{Zn}$ anodes are circumvents of $\mathrm{Zn}$ dendrite and the improvement of electron transfer as well as ionic diffusion. Compared with bare $\mathrm{Zn}$ foil, the coating protection and well-designed electrodepositing strategies of the $\mathrm{Zn}$ electrode enable them more appropriate for irreversible and high-efficient $\mathrm{Zn}$-ion depositing/ stripping processes in ZHSCs. Also, the recoverable $\mathrm{Zn}$ anode method can promote the lossless realization of renewable ZHSCs. We believe that a well-designed $\mathrm{Zn}$ anode should be focused on both the energy-part and power-part. The excellent integration of energy-part and power-part may facilitate the promotion of energy density in ZHSCs. 


\subsection{Cap Electrode}

Cap electrode material is a vital part for both two electrode configurations of ZHSCs, which can equip the ZHSCs with good conductivity, fast charge/discharge rate and high electrochemical stability. Among the reported ZHSCs, two kinds of Cap electrodes are investigated, including the carbon electrode and pseudocapacitive electrode. Typically, carbon electrodes like high specific surface area (HSSA) carbon electrode, heteroatom-doped carbon electrode and graphenebased electrode can deliver an excellent power performance based on the fast adsorption/desorption reactions and considerable capacity for ZHSCs. Pseudocapacitive electrodes such as MXenes, polymer-carbon hybrid electrodes and transition metal compounds (TMCs) also present high capacity with some unique properties for ZHSCs. In the following section, we will review recent progress of the Cap electrodes and corresponding electrochemical performance in ZHSCs.

\subsubsection{Carbon Electrode}

The carbon electrodes with splendid properties of low cost, high chemical stability and good conductivity have been regarded as the optimal Cap electrode candidates for highperformance ZHSCs [31, 101, 102]. By proceeding the fast ion adsorption/desorption processes in the ZHSCs, the carbon electrodes can store some energy and further deliver considerable power performance. Nevertheless, due to the energy storage mechanism of EDLC, the normal carbon electrodes cannot offer sufficient energy because of the limited specific surface area (SSA). Thus, many strategies were proposed to promote the energy and power performance of carbon electrodes, such as (1) HSSA carbon electrode based on active carbon (AC), porous carbons (PCs), hollow carbon spheres (HCSs), flower-like carbons (FCs) and CNTs electrodes, (2) heteroatom-doped carbon electrodes. Additionally, graphene-based electrode is also a promising carbon electrode for ZHSCs. In this section, various recently reported carbon electrodes are expressly reviewed.

2.2.1.1 HSSA Carbon Electrode As mentioned above, the carbon electrode is the representative cathode for $\mathrm{Zn} / / \mathrm{Cap}$ ZHSCs. Due to the energy storage mechanism of adsorption/desorption reaction, the capacity is highly connected with the SSA of electrode materials, thus, common strat- egies focus on implementing the HSSA carbon cathode [103-106]. The conventional AC materials were reported by Tang et al. and the corresponding Zn//AC ZHSCs were fully investigated [101]. To some extent, the AC is a microporous carbon material with HSSA. As shown in Fig. 4a, the rectangular shapes of $\mathrm{CV}$ curves demonstrated that the $\mathrm{Zn} / /$ AC ZHSCs have typical Cap energy storage behaviors, whereas the energy density $\left(52.7 \mathrm{Wh} \mathrm{kg}^{-1}\right)$ of the ZHSCs is much more than normal supercapacitors [107]. After 20,000 cycles of charging/discharging processes (Fig. 4b), the $\mathrm{Zn} / /$ AC ZHSCs maintained stable electrochemical behavior, and the capacity had no evident decline (remained $91 \%$ of initial capacity). Although the ultra-stable property of commercial AC materials provides the ZHSCs with ultra-long longevity, the energy density of as-prepared ZHSCs is not high enough for practical applications. Thus, Kang et al. optimized the electrochemical performance with AC powders and the $\mathrm{Zn} / / \mathrm{AC}$ powders ZHSCs offered a higher capacity of $84 \mathrm{Wh} \mathrm{kg}^{-1}$ [31]. Gratifyingly, the Zn//AC ZHSCs exhibited a splendid cycling performance (91\% after 10,000 cycles) at a very low current density of $1 \mathrm{~A} \mathrm{~g}^{-1}$, which is almost the battery-level cyclic condition but the longevity is ten times longer than batteries. Accompanying with the normal adsorption/desorption reaction, the precipitation and dissolution processes of by-products $\left(\mathrm{Zn}_{4} \mathrm{SO}_{4}(\mathrm{OH})_{6} \cdot 5 \mathrm{H}_{2} \mathrm{O}\right)$ is detected by the XRD spectra (Fig. 4c) of AC powders [31]. In addition, more by-products like $\mathrm{ZnSO}_{3} \cdot 2.5\left(\mathrm{H}_{2} \mathrm{O}\right)$ were demonstrated in various ZHSCs during the charging/discharging processes $[34,77,103]$. The effects and particular mechanisms of the by-products will be carefully discussed in Sect. 3.

Apart from the commercial AC, many PCs electrode materials can be fabricated through various methods, like template method [111-113] and pyrolysis method [108, 114-116]. As for the pyrolysis methods, the precursor is a significant part of synthesizing high-quality PC electrode materials. Lu et al. reported molten salt-assisted synthesis of pitch-derived PCs cathodes for Zn//Cap ZHSCs while Guan et al. proposed bamboo-derived PCs cathodes with superb stability when employed in ZHSCs (96\% capacity retention after 90,000 cycles) [114, 115]. Moreover, Xu et al. and Shen et al. reported a MOF route for fabricating PCs electrode materials, the ZIF-8-derived PCs presented an splendid performance of $225 \mathrm{mAh} \mathrm{g}^{-1}$ at $0.1 \mathrm{~A} \mathrm{~g}^{-1}$ and remained $135.5 \mathrm{mAh} \mathrm{g}^{-1}$ at $1 \mathrm{~A} \mathrm{~g}^{-1}[98,116]$. As depicted in Fig. 4d, Alshareef et al. reported a two-step synthesis method including pyrolysis at $800{ }^{\circ} \mathrm{C}$ and acid etching for manufacturing PC-800 cathode [108]. After then, the PC-800 cathode showed additional pseudocapacitance 

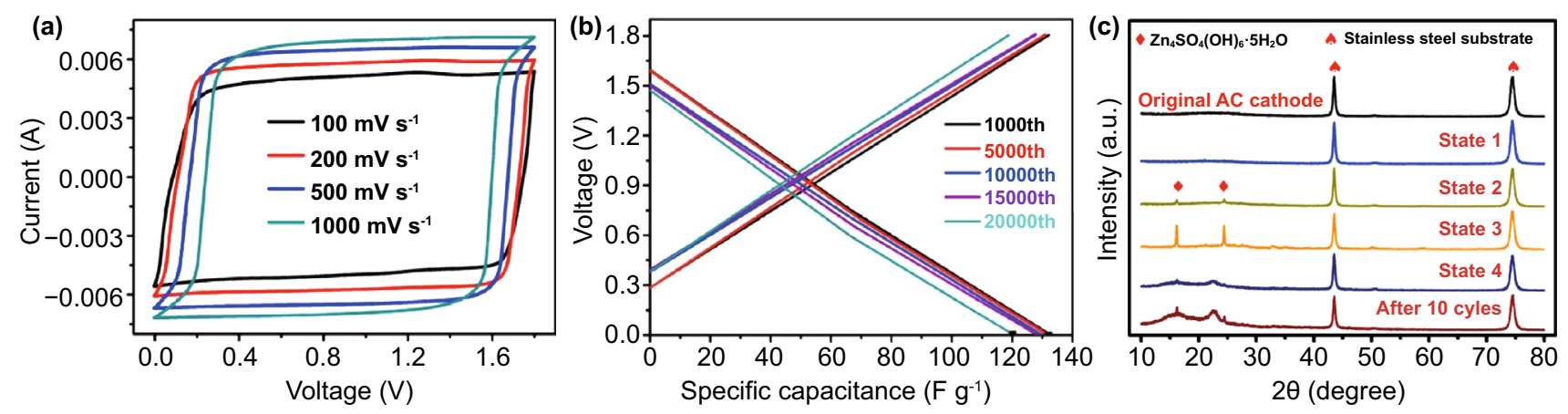

(d)
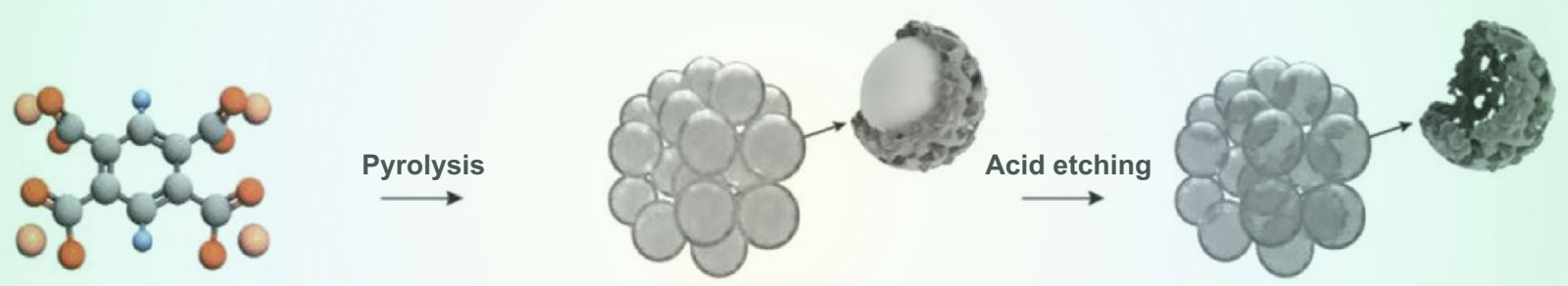

Pyromellitic acid tetrapotassium salt (PMA4K)

Porous carbon (PC)
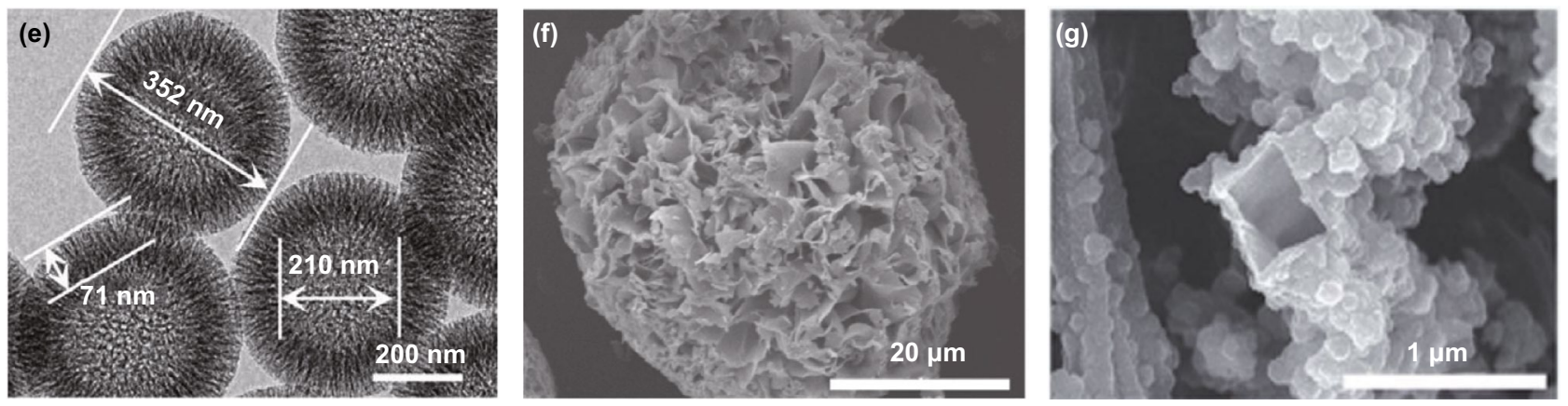

Fig. 4 a CV curves and b GCD profiles of representative Zn//AC ZHSCs. Reproduced with permission from Ref. [101]. Copyright 2018, Elsevier. c XRD patterns of AC electrodes at different states. Reproduced with permission from Ref. [31]. Copyright 2018, Elsevier. d Schematic diagram illustrating the synthesis processes of PCs. Reproduced with permission from Ref. [108]. Copyright 2020, Wiley-VCH. e SEM image of HCSs. Reproduced with permission from Ref. [92]. Copyright 2020, Elsevier. f SEM image of FCs. Reproduced with permission from Ref. [109]. Copyright 2021, Elsevier. g SEM image of CNTs. Reproduced with permission from Ref. [110]. Copyright 2019, Royal Society of Chemistry

which are originated from the variation in oxidation states of oxygen functional groups. The as-assembled $\mathrm{Zn} / /$ PC-800 ZHSCs reached a high capacity of $179.8 \mathrm{mAh} \mathrm{g}^{-1}$ and surprising capacity retention of $99.2 \%$ after 30,000 cycles.

HCSs, which possess hollow structures and micro- or nano-shells, are regarded as a promising HSSA carbon cathode as well. The hollow structure enables the HCSs cathode great specialties of high surface-to-volume ratio and substantial accessible sites. Chen et al. presented the HCSs cathode through the calcination of co-polymer and applied a surfactant as the soft template [99]. After that, the HCSs cathode matched with a flexible Zn@CC anode to assemble Zn@CC//HCSs ZHSCs. The HCSs have a uniform size of $\sim 100 \mathrm{~nm}$ external diameter and $\sim 50 \mathrm{~nm}$ inner diameter, and the Brunauer-Emmett-Teller (BET) surface area of HCSs was measured to be $819.5 \mathrm{~m}^{2} \mathrm{~g}^{-1}$. Benefiting from the HSSA property and hollow structure of HCSs, the ZHSCs achieved energy density of $59.7 \mathrm{Wh} \mathrm{kg}^{-1}$ in a low concentration of $0.08 \mathrm{M} \mathrm{ZnSO}_{4}$ polyacrylamide (PAM) hydrogel electrolyte and ultra-stable cycling performance $(98 \%$ of 
initial capacity) after 15,000 cycles. Due to the low concentration and solid-state property of the applied electrolyte, the energy density of the flexible Zn@CC//HCSs ZHSCs is limited compared to aqueous ZHSCs. To further investigate the performance of HCSs cathode in the aqueous ZHSCs, Liu et al. demonstrated MCHSs cathodes with ultra-high BET surface area of $1275 \mathrm{~m}^{2} \mathrm{~g}^{-1}$ [92]. As shown in Fig. 4e, the external and inner diameters of MCHSs are 352 and $210 \mathrm{~nm}$, respectively. With the increasing mass loading of electrodes, the HSSA carbon cathode may suffer from worsening ion and charge transport characteristics in thicker electrodes. However, the interconnected porous of MCHSs can provide a short ion and charge transport path as well as good penetration, leading to its excellent electrochemical performance of ZHSCs even under the condition of high mass loadings. Hence, the as-assembled ZHSCs can deliver a remarkable energy density of $129.3 \mathrm{Wh} \mathrm{kg}^{-1}$ and $100 \%$ capacity retention after 1000 cycles at $0.1 \mathrm{~A} \mathrm{~g}^{-1}$. When increasing the current density and mass loading, the MCHS-based ZHSCs only showed a small capacity degradation.

Recently, a new HSSA carbon cathode of FCs was applied by $\mathrm{Hu}$ et al. as the carbon cathode for $\mathrm{Zn} / / \mathrm{Cap} \mathrm{ZHSCs}$ [109, 117]. The hierarchical 3D FCs are formed by the spheroidizing growth processes of carbon and low-dimensional carbon nanosheets, which possess large surface area, large surface-to-volume ratios, interconnected conductive network, nanoscale architecture and abundant accessible active sites. The SEM shown in Fig. 4f exhibited that the diameter of the $3 \mathrm{D}$ FCs is $\sim 40 \mu \mathrm{m}$ and the size of the carbon nanosheets is $\sim 2 \times 2 \mu \mathrm{m}^{2}$. Based on the unique structure of 3D FCs, the $\mathrm{Zn} / / \mathrm{FC}$ ZHSCs offered $119.7 \mathrm{Wh} \mathrm{kg}^{-1}$ at $890 \mathrm{~W} \mathrm{~kg}^{-1}$ and splendid cycling performance of $92 \%$ capacity retention after 20,000 cycles. In addition, 1D CNTs displayed in Fig. 4g can also be used as HSSA carbon electrodes for ZHSCs depend on their special property of decent electrical conductivity, splendid mechanical property, high chemical stability and uniform distribution of pore size [118, 119]. Although the reported CNTs electrodes possess a low SSA of 200-300 $\mathrm{m}^{2} \mathrm{~g}^{-1}$ compared with PCs and HCSs electrodes, the CNT-based carbon electrode can offer incredible capacity for ZHSCs [110, 120, 121]. Han et al. applied $\mathrm{B}, \mathrm{N}$ co-doped porous carbon microtube (BN-CMTs) as the cathode of ZHSCs and ultimately achieve an ultrahigh capacity of $472.6 \mathrm{Wh} \mathrm{kg}^{-1}$ [110]. Moreover, research showed that the electrochemical characteristics of CNTs can be highly improved by many activation methods depending on their intrinsic properties $[119,122]$. Based on the excellent mechanical property, Chen et al. applied CNTs as the flexible substrate for both cathode and anode materials, assembling a highly flexible fiber-type ZHSCs [120]. The as-prepared ZHSCs can keep similar CV curves under bent and twisted conditions, delivered an outstanding specific capacity of $104.5 \mathrm{~F} \mathrm{~cm}^{-3}$ and steady cycling performance of $98.5 \%$ capacity retention after 10,000 cycles.

After reviewing the reported HSSA carbon electrode of ZHSCs, one phenomenon can be discovered that the relationship between capacity and surface area is not linear. Two HSSA carbon electrodes with similar BET surface area and configuration of ZHSCs may offer disparate energy density. From our point of view, it can be ascribed to overlapping effects and electronic kinetics. Although the HSSA carbon electrodes are achieved by various nanostructures, the effective solution-surface contact area varies from structure to structure. Sometimes, the inside carbon materials can be shielded by external carbon materials, thus leading to invalid surface area for EDLC. Similarly, the electronic kinetics are also affected by different surface morphology and mass loading of carbon electrodes. Hence, the obsession for extremely high SSA carbon electrodes is inefficient and counter-productive. On the contrary, prominent nanostructure and modified component of carbon electrode with suitable SSA may be the way for realizing higher performance ZHSCs.

2.2.1.2 Heteroatom-Doped Carbon Electrode Particularly, heteroatom doping is an efficient way to modify the physical and chemical properties of carbon electrodes [123125]. By replacing part of $C$ atoms with other elements like $\mathrm{B}, \mathrm{N}$ and $\mathrm{P}$, the strategy can not only optimize the synthesis process of heteroatom-doped carbon electrodes but also further improve the electrochemical performance of the as-prepared ZHSCs [126]. For one, heteroatom doping can improve the electrical conductivity, create more electrolyte accessible active sites and alleviate agglomeration of carbon electrodes [127]. For another, heteroatom doping can accelerate the permeation and diffusion character of electrolytes, thus further promote the wettability of carbon electrodes $[128,129]$.

Accordingly, Lu group reported that the $\mathrm{N}$ doping is expected to favor the chemical absorption of $\mathrm{Zn}$-ions and tremendously altered the properties like conductivity, surface wettability, electrochemically active surface area as well as ion/electron transportation of original PCs [85]. The synthesis illustration of HNPC is shown in Fig. 5a. Particularly, 


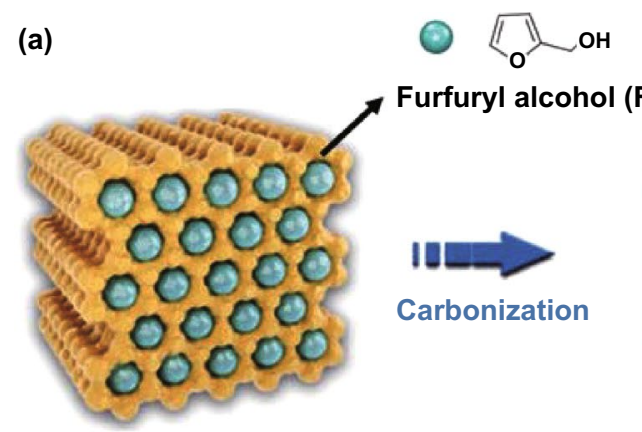

FA in NaY template

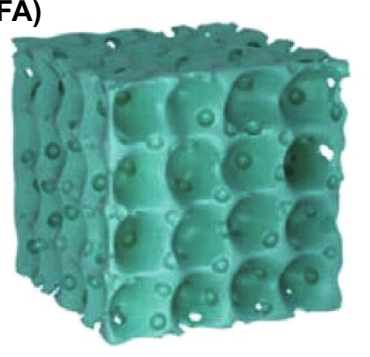

PC
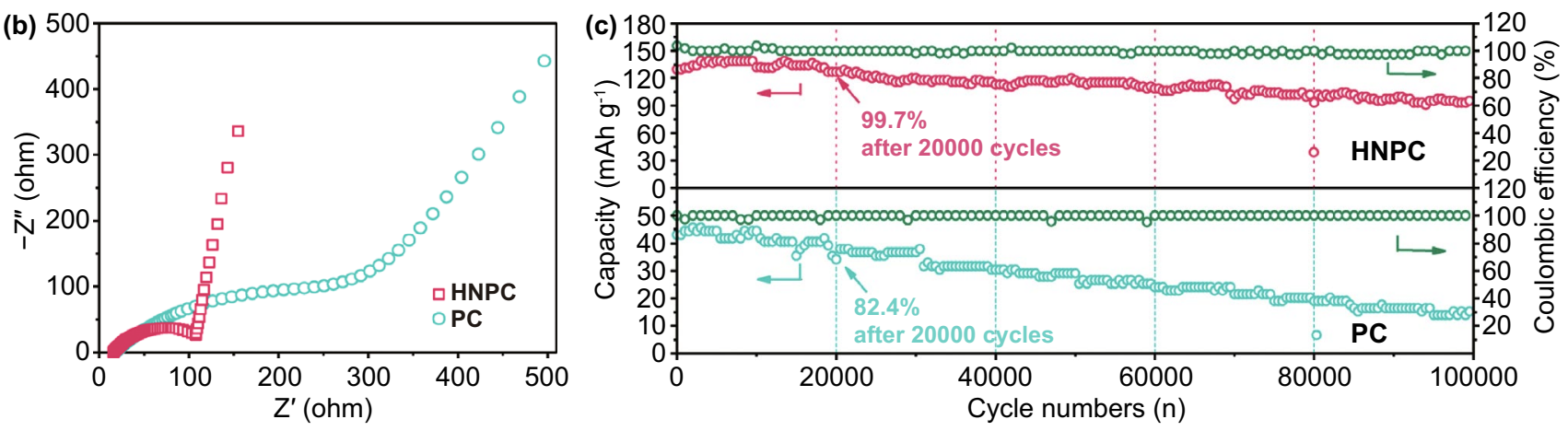

(d)

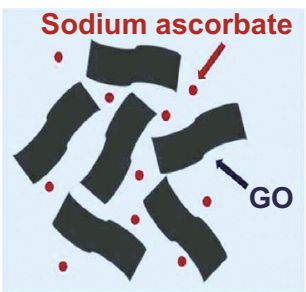

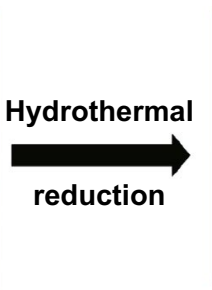

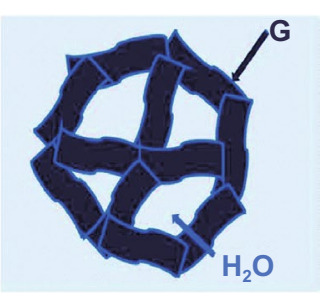

(e)

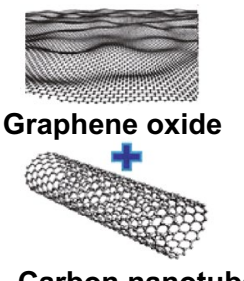

HNPC

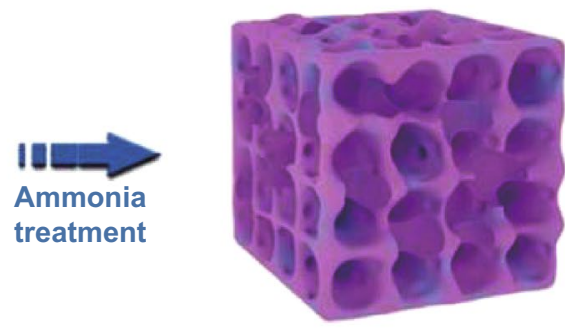

treatment

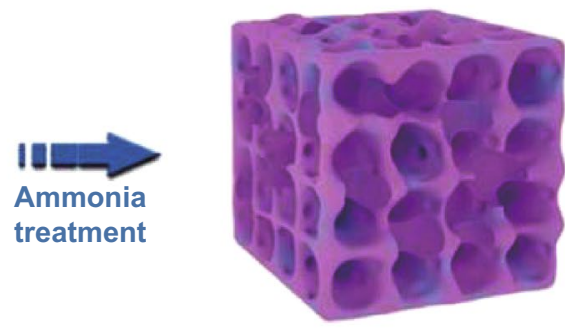

(f)

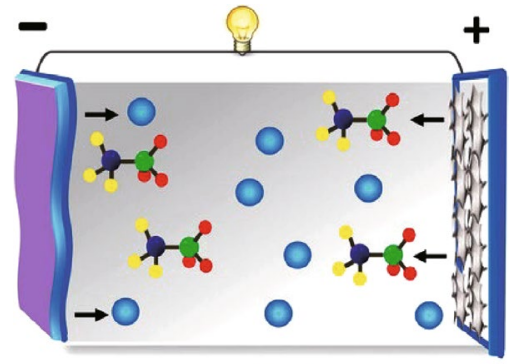

$\mathrm{Zn}$ foil $\mathrm{Zn}^{2+} \mathrm{O}-\mathrm{CF}_{3} \mathrm{SO}_{3}^{-}$aMEGO

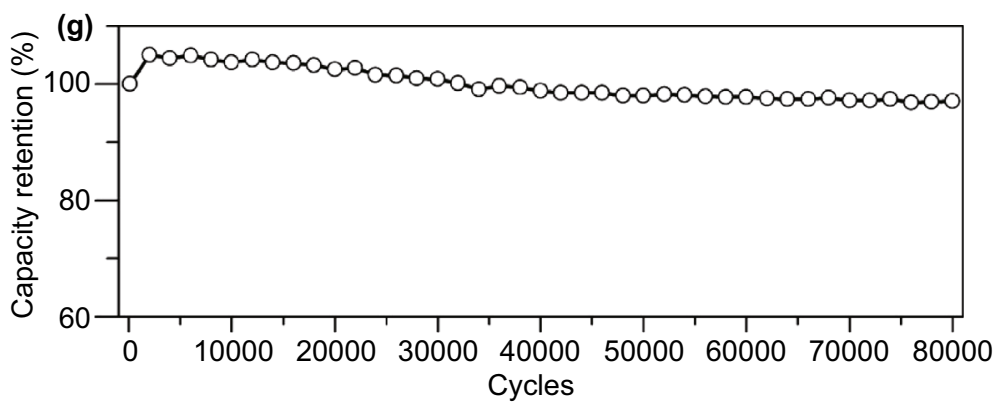

Fig. 5 a Schematic illustration of the synthesis route of the HNPC. b Nyquist plots and c cycling performance at $16.7 \mathrm{~A} \mathrm{~g}-1$ of the $\mathrm{Zn} / / \mathrm{PC}$ and Zn//HNPC ZHSCs. Reproduced with permission from Ref. [85]. Copyright 2019, Wiley-VCH. d Schematic of the preparation process of 3D graphene by hydrothermal reduction. Reproduced with permission from Ref. [131]. Copyright 2018, Royal Society of Chemistry. e Schematic illustration of the synthesis of rGO/CNT. Reproduced with permission from Ref. [120]. Copyright 2019, Wiley-VCH. f Schematic illustration of discharging process and $\mathbf{g}$ cycling performance of the Zn//aMEGO ZHSCs. Reproduced with permission from Ref. [82]. Copyright 2019, Wiley-VCH

the HNPC-based ZHSCs can offer an ultra-high capacity of $177.8 \mathrm{mAh} \mathrm{g}^{-1}$, which is almost three times higher than non-N-doped PC and higher than most carbon-based electrodes. Through the EIS tests shown in Fig. 5b, the fantastic promotion of N-doping strategy in decreasing charge transfer resistance and enhancing the diffusion rate was demonstrated compared with original PCs electrodes. Furthermore, 
the strategy of heteroatoms doping can even boost the stability of HNPC electrodes. As depicted in Fig. 5c, the HNPC-based ZHSCs maintained $99.7 \%$ capacity retention after 20,000 cycles while the PC-based ZHSCs displayed a lower capacity retention of $82.4 \%$. Liu group also proposed an N-doped hierarchical porous carbon (N-HPC) which is derived from commercial chitosan via simultaneous carbonization and activation [130]. Gratifyingly, a remarkable energy density of $191 \mathrm{Wh} \mathrm{kg}^{-1}$ was achieved by N-HPCbased ZHSCs.

Apart from $\mathrm{N}$ atoms, $\mathrm{P}$ dopant has been reported in the carbon electrodes of ZHSCs as well, which possesses a lower electronegativity of 2.19 than $\mathrm{N}$ atoms [127]. Chen et al. prepared P-doped honeycomb-like porous carbon (PHCA) electrodes by replacing the $\mathrm{C}$ atoms in carbon skeletons with P dopant [112]. Subsequently, the P dopants in carbon skeletons lead a chemical bonds transformation from $\mathrm{C}-\mathrm{OH}$ (C-C-OH) to $\mathrm{P}-\mathrm{C}-\mathrm{OH}$, which results in a stronger polarization of the hydroxyl group and lower energy barrier (from 0.13 to $0.04 \mathrm{eV}$ ). The stronger polarization of the hydroxyl group and lower energy barrier further enable a more attainable reaction during charging/discharging processes. Based on the PHCA electrodes, the Zn//PHCA ZHSCs presented a peak energy density of $129.3 \mathrm{Wh} \mathrm{kg}^{-1}$ at a power density of $1000 \mathrm{~W} \mathrm{~kg}^{-1}$.

Besides the single heteroatom-doping strategy, co-doping strategy will further promote excellent performances of carbon electrodes based on the synergistic effect of diverse heteroatoms. $\mathrm{N}, \mathrm{O}$ co-doped hierarchical porous carbon (NO-HPC) was employed as the cathode in ZHSCs by Zhao group, where the ZIF-8 is applied as the template and C source [132]. Although the SSA of the carbon electrode is relatively low $\left(197.45 \mathrm{~m}^{2} \mathrm{~g}^{-1}\right)$, the $\mathrm{Zn} / / \mathrm{NO}-\mathrm{HPC}$ ZHSCs exhibited splendid energy density of $110 \mathrm{Wh} \mathrm{kg}^{-1}$ and lossless capacity after 10,000 cycles. In addition, P, B co-doped AC (PB-AC) is reported by An et al. and the $\mathrm{Zn} / /$ PB-AC ZHSCs delivered a high energy density of 169.4 $\mathrm{Wh} \mathrm{kg}^{-1}$ and superb longevity of 30,000 cycles [133]. Furthermore, the second-highest energy density $(472.6 \mathrm{Wh}$ $\mathrm{kg}^{-1}$ ) of reported ZHSCs is based on the $\mathrm{B}, \mathrm{N}$ co-doped BN-CMTs electrodes, which is even higher than most of ZIBs and LIBs [110]. However, the SSA of the BN-CMTs $\left(101.24 \mathrm{~m}^{2} \mathrm{~g}^{-1}\right)$ is much lower than most HSSA carbon electrodes mentioned above. It is believed that the $\mathrm{B}, \mathrm{N}$ co-doping strategy facilitates the faradaic reactions of pseudocapacitance on the electrode surface by changing the electronic structure and density state of carbon, thus realizing prominent energy density even under a relatively low SSA. In short, heteroatom doping is an effective and promising strategy for enhancing the electrochemical performance of carbon electrodes. However, the deep working mechanisms of carbon electrodes heteroatom doping in ZHSCs are still in need. A better understanding of heteroatom doping in ZHSCs is highly significant, which leads to reasonable dopants and reproducible high performance of ZHSCs.

2.2.1.3 Graphene-Based Electrode In addition to normal active carbon materials, graphene is the most common material used in symmetric supercapacitors [134]. As a novel 2D material with excellent properties such as decent intrinsic electric conductivity in-plane, high theoretical SSA of 2630 $\mathrm{m}^{2} \mathrm{~g}^{-1}$, outstanding mechanical strength and chemical stability, graphene and its derivatives have been widely applied in many ESSs [134-136]. The intrinsic capacitance of monolayer graphene can reach $\sim 21 \mu \mathrm{F} \mathrm{cm} \mathrm{cm}^{-2}$ according to its theoretical SSA [137]. Also, graphene-based materials are characterized by ultra-low RC constants of less than $200 \mu \mathrm{s}$, while the number is about $1 \mathrm{~s}$ for typical carbon materials [138]. Hence, it is generally believed that graphene-based materials is a promising candidate for high-performance ZHSCs.

As shown in Fig. 5d, Han et al. fabricated graphene-based electrodes for ZHSCs, where the reduced graphene oxide electrodes (G) are derived from the graphene oxide (GO) via hydrothermal reduction [131]. After further modified the $\mathrm{G}$ electrodes with polyaniline (PANI) by novel in-situ polymerization, the highest energy density (205 $\mathrm{Wh} \mathrm{kg}^{-1}$ ) in graphene-based ZHSCs was achieved. However, the G electrodes without further polymerization showed a better cycling performance than G@PANI electrodes, indicating the trade-off between energy density and cycling stability. As a novel material, graphene was widely employed for the modification of other carbon electrodes in ZHSCs. For instance, Wang et al. and Xu et al. improved the cycling performance of ZHSCs by introducing graphene materials into the electrodes $[139,140]$. As illustrated in Fig. 5e, Chen group demonstrated a flexible fiber-shaped reduced graphene oxide (rGO) on CNT substrate by capillary hydrothermal method [120]. Moreover, the rGO//CNT cathode was coupled with the electrodeposited $\mathrm{Zn} / /$ graphene fiber anode to form ZHSCs. Benefiting from the both graphene-based electrodes, the flexible ZHSCs device delivered a splendid 
energy density of $48.5 \mathrm{mWh} \mathrm{cm}^{-3}$ and decent longevity of 10,000 cycles with merely $1.7 \%$ capacity decay.

Furthermore, the ultra-high stability of graphene-based electrodes in ZHSCs was confirmed by various investigations. For one, Wang et al. developed a porous 3D MXenesrGO aerogel cathode for ZHSCs with an ultra-long cycling lifetime (95\% capacity retention after 75,000 cycles) [139]. The introduced MXenes $\left(\mathrm{Ti}_{3} \mathrm{C}_{2} \mathrm{~T}_{x}\right)$ in the composite electrodes can provide an additional pseudocapacitive reaction of $\mathrm{Zn}$-ion insertion/desertion, resulting in an improvement of ZHSCs capacity. For another, Zhang group fabricated $\mathrm{PC}$ electrodes derived from chemical activated microwave exfoliated graphene oxide (aMEGO) via three-step process of microwave treatment, $\mathrm{KOH}$ activation and washingdrying-annealing [134]. As depicted in Fig. 5f, the structure of $\mathrm{Zn} / /$ aMEGO ZHSCs is illustrated [82]. By applying 3.0 M Zn( $\left(\mathrm{CF}_{3} \mathrm{SO}_{3}\right)_{2}$ and $\mathrm{Zn}$ foil as the electrolyte and anode, respectively, the $\mathrm{Zn} / / \mathrm{aMEGO} \mathrm{ZHSCs}$ are assembled and provided a battery-level energy density of $106.3 \mathrm{Wh} \mathrm{kg}^{-1}$ and a capacitor-level power density of $31,400 \mathrm{~W} \mathrm{~kg}^{-1}$. Furthermore, it can achieve incredible stability that remained $93 \%$ of initial capacity after 80,000 charging/discharging cycles at $8 \mathrm{~A} \mathrm{~g}^{-1}$ (Fig. 5g). These two cases are tested in different electrolytes where the former is $2.0 \mathrm{M} \mathrm{ZnSO}_{4}$ and the latter is $3.0 \mathrm{M} \mathrm{Zn}\left(\mathrm{CF}_{3} \mathrm{SO}_{3}\right)_{2}$. Hence, the general adaptability and stability of graphene-based materials in ZHSCs are prominent, and the graphene-based electrodes are proved to be a great choice for high-performance ZHSCs.

\subsubsection{Pseudocapacitive Electrode}

Although featured with HSSA, special morphologies and splendid electrical conductivity, the energy densities of most pure carbon electrodes are still far from practical applications due to the limited energy storage mechanism of EDLC based on simple physical adsorption/desorption processes. By contrast, pseudocapacitive materials with the co-existence of reversible faradic reactions and EDLC are promising candidates for large capacity of ZHSCs [141, 142]. As we mentioned above, the heteroatom-doped carbon material is a typical pseudocapacitive material for ZHSCs, and the pseudocapacitive mechanisms have been already discussed. Except the heteroatom-doped carbon materials, many carbon-based composite electrodes combined with carbon materials and pseudocapacitive materials can present noteworthy pseudocapacitance, however, have not been applied for ZHSCs [143]. As the important component of pseudocapacitive materials, we believed that more and more carbon-based pseudocapacitive materials with composite energy storage mechanisms will be investigated in the system of ZHSCs, and then lead to high-performance ZHSCs. In the following section, we are going to discuss some reported works of pseudocapacitive electrodes such as MXenes, polymer-carbon hybrid materials and TMCs (e.g., $\mathrm{RuO}_{2}, \mathrm{TiN}$ ), and analyze the corresponding electrochemical performance of as-prepared ZHSCs.

2.2.2.1 MXenes MXenes, the prominent family of $2 \mathrm{D}$ transition metal carbide and nitrides, are widely investigated and employed in many ESSs like LIBs and supercapacitors [26, 144-147]. It is generally demonstrated that the MXenes electrodes can be hosts for a range of cations such as $\mathrm{Na}^{+}, \mathrm{K}^{+}, \mathrm{NH}_{4}^{+}, \mathrm{Mg}^{2+}$ and $\mathrm{Zn}^{2+}[148,149]$. Additionally, the ions penetrations only occur between the MXene sheets because the chemical bonds between $\mathrm{M}$ and $\mathrm{X}$ are unbreakable, thus occupying electrochemically active sites on the MXenes surfaces and realizing energy storage [150, 151]. Although the CV curves of MXenes electrodes are rectangular shapes without pronounced redox peaks, the energy storage mechanism of MXenes in acid is prominently proved to be pseudocapacitive [152]. As a promising candidate of pseudocapacitive cathode for ZHSCs, MXenes have attracted numerous attentions. Hence, some currently reported researches of MXenes on ZHSCs will be reviewed as follows.

Among the family of MXenes, the $\mathrm{Ti}_{3} \mathrm{C}_{2} \mathrm{~T}_{x}$ is the most studied MXene for capacitors, which can achieve outstanding capacities of 300-400 $\mathrm{F} \mathrm{cm}^{-3}$, surpassing most all-carbon-based super capacitors [148, 152]. Accordingly, Zhi group applied $\mathrm{Ti}_{3} \mathrm{C}_{2}$ electrode as the cathode of ZHSCs and further fabricated a flexible ZHSCs with the wholly degradable property [96]. The capacity of Zn@ $\mathrm{Ti}_{3} \mathrm{C}_{2} / / \mathrm{Ti}_{3} \mathrm{C}_{2}$ ZHSCs is enhanced to $330 \mathrm{~F} \mathrm{~cm}^{-3}$, which can be ascribed to the abundant pseudocapacitive reactions. Notably, the $\mathrm{Zn}-\mathrm{Ti}_{3} \mathrm{C}_{2}$ ZHSCs can totally degrade within 7 days (Fig. 3c, d), which drastically exceeding the current supercapacitors of $\sim 120$ days and batteries of $\sim 20$ days. However, the decent degradable performance is realized at the cost of cycling longevity due to the relatively low thickness of $\mathrm{Zn}-\mathrm{Ti}_{3} \mathrm{C}_{2}$. Therefore, the $\mathrm{Zn} @ \mathrm{Ti}_{3} \mathrm{C}_{2} / / \mathrm{Ti}_{3} \mathrm{C}_{2}$ ZHSCs can only maintain capacity retention of $82.5 \%$ after 1000 cycles. To promote the longevity of $\mathrm{Ti}_{3} \mathrm{C}_{2}$-based ZHSCs, many efforts have been devoted. As we reviewed 


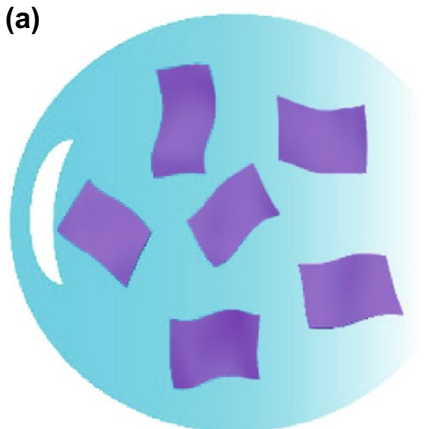

$\mathrm{Ti}_{3} \mathrm{C}_{2} \mathrm{~T}_{\mathbf{x}}$ dispersion

(b)
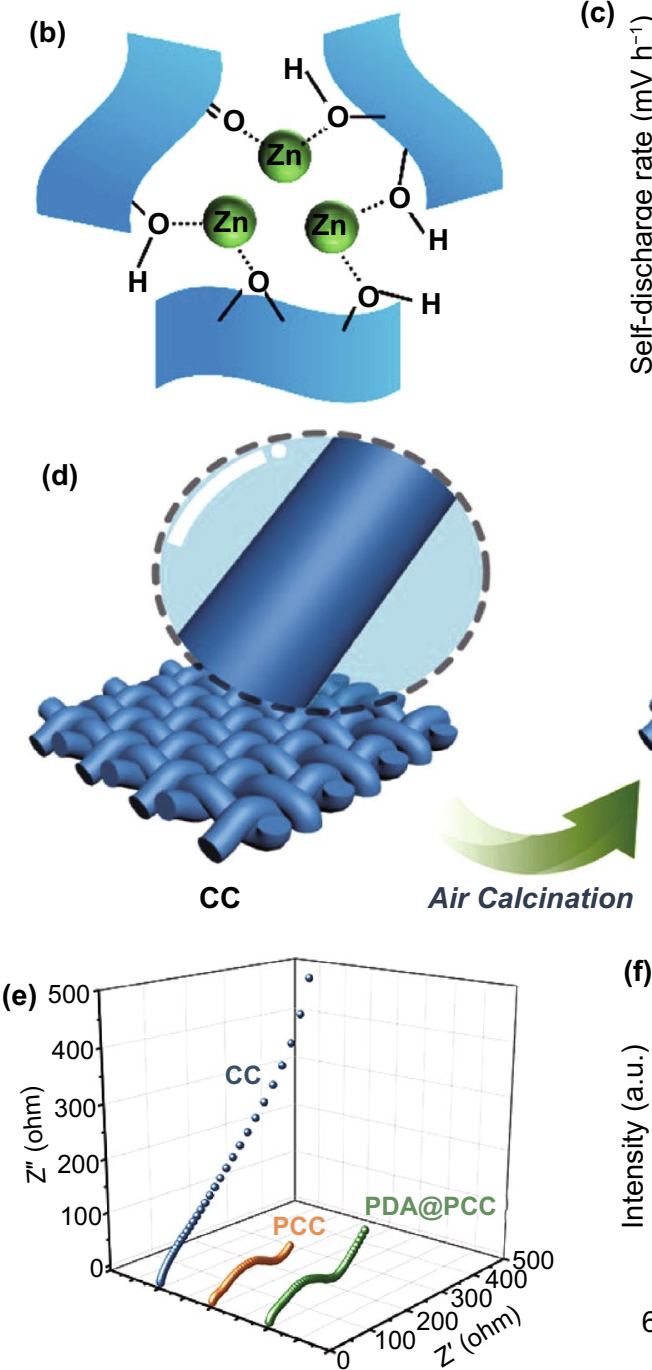

(d)
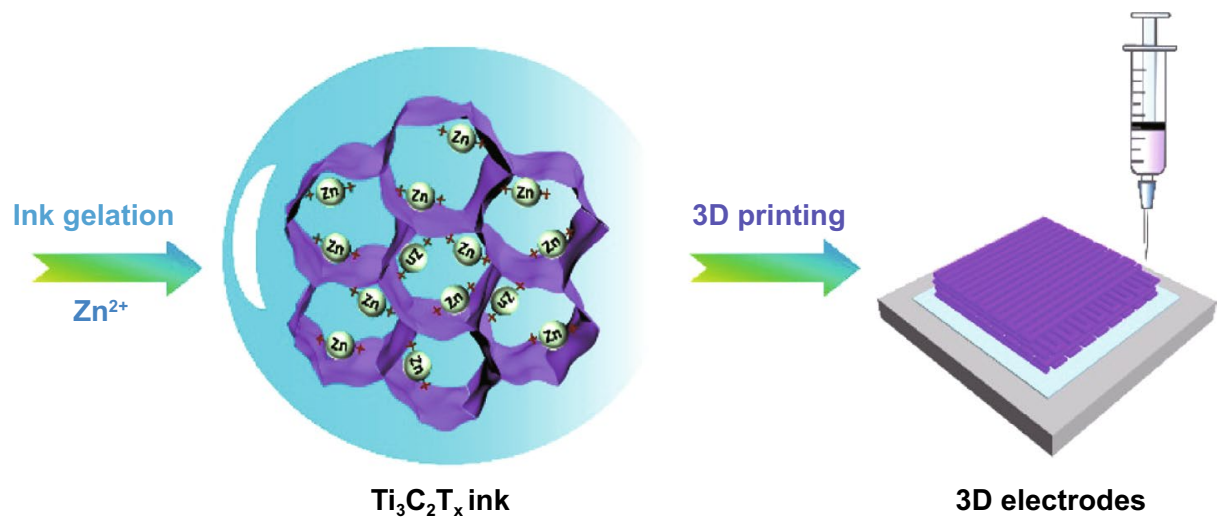

3D electrodes (c)

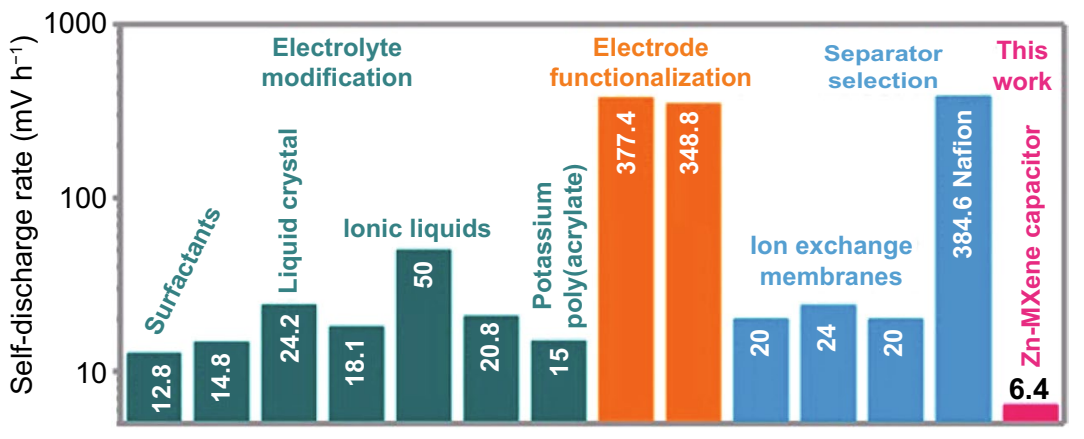

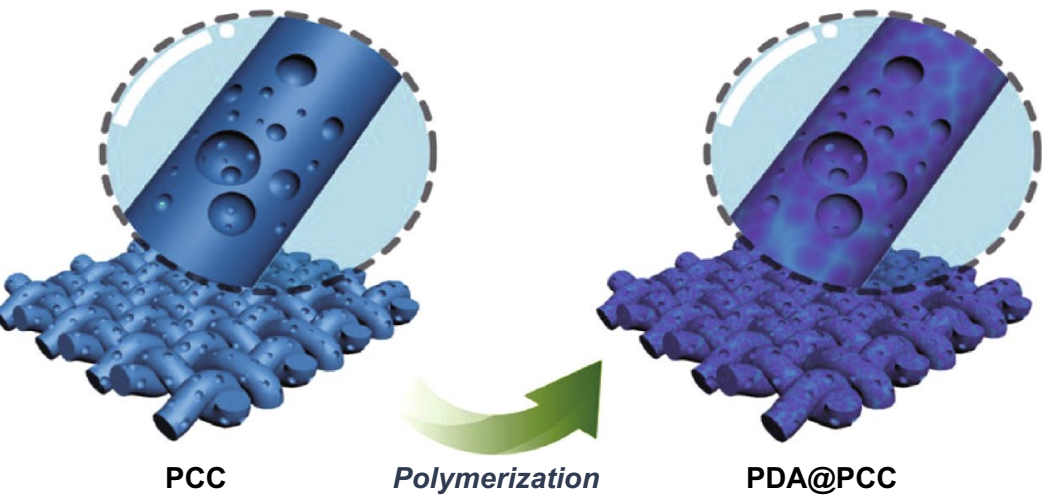
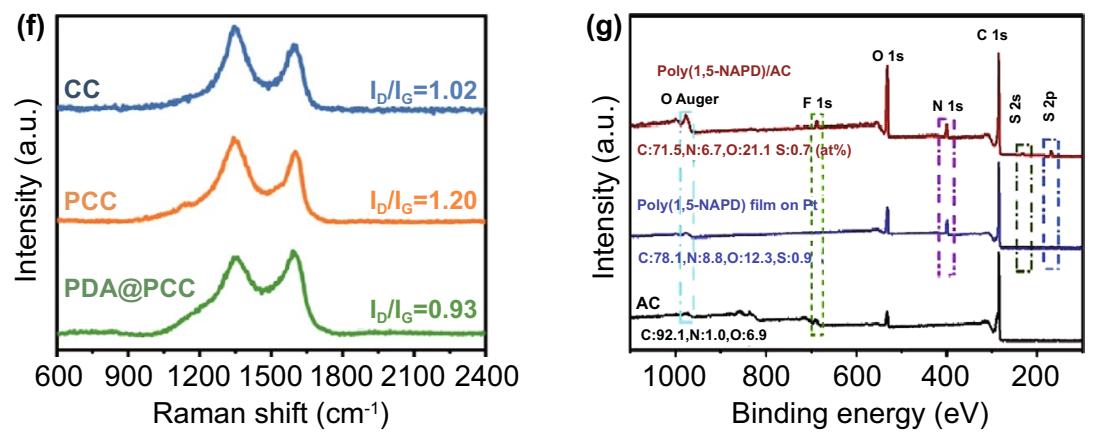

Fig. 6 a Illustration of MXenes ink formulation and the 3DP process. b Schematic illustration of the Zn-ion gelation process targeting the preparation of MXenes ink. Reproduced with permission from Ref. [91]. Copyright 2021, American Chemical Society. c Comparison of selfdischarge rate between the $\mathrm{Zn} / / \mathrm{MXenes}$ capacitor and previously reported anti-discharge supercapacitors. Reproduced with permission from Ref. [96]. Copyright 2019, American Chemical Society. d Preparation process of the PDA@PCC electrode. e Nyquist plots and f Raman spectra of CC, PCC and PDA@ PCC electrodes. Reproduced with permission from Ref. [154]. Copyright 2020, American Chemical Society. g XPS spectra of poly(1,5-NAPD)/AC cathode, poly(1,5-NAPD) film on Pt, and AC coating. Reproduced with permission from Ref. [77]. Copyright 2020, Elsevier 
above, graphene-based ZHSCs showed superb cycling performance based on the extraordinary chemical stability. Therefore, Wang et al. prepared an MXenes-rGO electrode with the both excellent performance of MXenes and rGO [139]. The Zn//MXenes-rGO ZHSCs presented a prolonged cycling lifetime of 75,000 cycles and outstanding capacity retention of $95 \%$. Furthermore, an in-situ annealing strategy is employed by Shen group to optimize the cycling performance of $\mathrm{Ti}_{3} \mathrm{C}_{2} \mathrm{~T}_{x}$-based ZHSCs [153]. Before the annealing, the $\mathrm{Ti}_{3} \mathrm{C}_{2} \mathrm{~T}_{x}$-based ZHSCs remained only $\sim 54.7 \%$ of initial capacity after 5000 cycles. However, after annealed at $300{ }^{\circ} \mathrm{C}$ for $30 \mathrm{~min}$ (Ar atmosphere), the $\mathrm{Ti}_{3} \mathrm{C}_{2} \mathrm{~T}_{x}$ electrodes presented ultra-long lifetime and outstanding stability ( $~ 80 \%$ after 50,000 cycles), which can be attributed to two reasons: (1) surface treatment of oxygen-containing functional group, (2) the generation of micropores in the $\mathrm{Ti}_{3} \mathrm{C}_{2} \mathrm{~T}_{x}$ structure.

As shown in Fig. 6a, Sun group reported a novel synthesis process of $\mathrm{Ti}_{3} \mathrm{C}_{2} \mathrm{~T}_{x}$ electrodes by $3 \mathrm{DP}$ technology [91]. The free-design property of 3DP technology enables more possibilities of $\mathrm{Ti}_{3} \mathrm{C}_{2} \mathrm{~T}_{x}$ electrodes including structure, thickness, size, etc. Significantly, the $\mathrm{Ti}_{3} \mathrm{C}_{2} \mathrm{~T}_{x}$ ink is the key part for 3DP technology, as depicted in Fig. 6b, the rheological behavior of $\mathrm{Ti}_{3} \mathrm{C}_{2} \mathrm{~T}_{x}$ ink is altered via introducing the $\mathrm{Zn}$-ion gelation. The electrostatic attraction between $\mathrm{Zn}^{2+}$ and negative charges on $\mathrm{Ti}_{3} \mathrm{C}_{2} \mathrm{~T}_{x}$ further decrease the repulsion of adjacent $\mathrm{Ti}_{3} \mathrm{C}_{2} \mathrm{~T}_{x}$ sheets. When assembled into the ZHSCs, the $3 \mathrm{DP} \mathrm{Ti}_{3} \mathrm{C}_{2} \mathrm{~T}_{x}$-based ZHSCs provide a splendid capacity of $1006.4 \mathrm{mF} \mathrm{cm}^{-2}$ at $0.1 \mathrm{~A}$ $\mathrm{g}^{-1}$ and rate capability (remain $71 \%$ when the current density up to $10 \mathrm{~A} \mathrm{~g}^{-1}$ ). Moreover, laser writing was applied by $\mathrm{Li}$ et al. to prepare $\mathrm{Ti}_{3} \mathrm{C}_{2} \mathrm{~T}_{x}$ electrodes, which is facile and matched with micro ZHSCs technology [153].

Furthermore, it is well known that self-discharge behavior is common and fatal for supercapacitors [155]. Lu et al. investigated the self-discharge phenomenon of BN-LDC// $\mathrm{Zn}$ ZHSC devices, where the devices can retain a high voltage of $1.4 \mathrm{~V}$ after $24 \mathrm{~h}$ self-discharge compared to the initial voltage of $1.8 \mathrm{~V}$, offering a fast self-discharge rate of $16.7 \mathrm{mV} \mathrm{h}^{-1}$ [83]. However, as shown in Fig. 6c, Zhi et al. prepared $\mathrm{Zn} @ \mathrm{Ti}_{3} \mathrm{C}_{2} / / \mathrm{Ti}_{3} \mathrm{C}_{2} \mathrm{ZHSCs}$ with lowest selfdischarge rate of $6.4 \mathrm{mV} \mathrm{h}^{-1}$ (from 1.35 to $0.89 \mathrm{~V}$ after $72 \mathrm{~h}$ ) among various supercapacitors which possess specific anti-self-discharge modification toward the electrode, electrolyte and separator [96, 156-159]. The key to this excellent behavior lies at the tight fixation between reduced $\mathrm{Zn}$ atoms and the anodic surface of MXenes electrodes under opencircuit state, thus, the $\mathrm{Zn}$ atoms will not transfer to cathodic area, leading to the remarkable anti-self-discharge property of MXenes. Although numerous advantages MXenes have shown, the operating voltage window of MXenes-based ZHSCs is quite narrow $(0-1.4 \mathrm{~V})$, which is limited for practical applications. Therefore, more efforts should be made to promote the higher operating voltage and better cycling performance of MXenes in the future.

2.2.2.2 Polymer-Carbon Hybrid Electrode Redox-active conducting polymers are also decent pseudocapacitive materials featured with fast and reversible redox reactions, thus the conducting polymer-based supercapacitors are able to provide higher capacity than those EDLC-only Cap materials. However, conducting polymer electrodes also cannot satisfy the longevity demand of high-performance supercapacitors due to the poor stability, active material loss and over-oxidative degradation during charging/discharging processes [160]. Hence, lots of studies try to solve the problem with the polymer-carbon hybrid electrode, where the ultra-stable carbon materials act as the substrate and the conducting polymer materials act as the active mass loadings. The excellent synergetic effect enables the polymercarbon hybrid materials to become suitable electrodes for ZHSCs.

Gratifyingly, many high-performance ZHSCs based on polymer-carbon hybrid electrodes have been reported recently, including different carbon substrates like CC, AC and rGO [77, 140, 154]. Han et al. employed PANI and rGO as the component of polymer-carbon hybrid electrodes and applied aniline solution on the surface of graphene hydrogel for the in-situ polymerization method [131]. Surprisingly, the PANI@rGO-based ZHSCs presented a capacity of $154 \mathrm{mAh} \mathrm{g}^{-1}$, which is five times higher than individual rGO electrodes. However, the PANI@rGO-based ZHSCs displayed an inferior capacity retention of $80.5 \%$ after 6000 charging/discharging cycles. Similarly, Hu group modified the rGO materials with another conducting polymer of p-phenylenediamine (PPD), where the PPD is inserted into the interlayer of rGO, resulting in bigger interlayer spacing and thus expose more ion accessible sites [140]. The PPD@ rGO hybrid electrode is fabricated by facial thermal evaporation and hydrothermal process. As a result, PPD@rGObased ZHSCs presented an incredible capacity of $3012.5 \mathrm{mF}$ $\mathrm{cm}^{-2}$ at a current density of $1 \mathrm{~mA} \mathrm{~cm}{ }^{-2}$, which is 11.2 times higher than the rGO-based ZHSCs provided. The excellent 
cycling performance of $100 \%$ capacity retention after 4000 cycles was realized by PPD@ rGO-based ZHSCs as well. Furthermore, Liu et al. prepared a poly(1,5-naphthalenediamine, NAPD)/AC hybrid electrode via a direct electrodepositing process [77]. Interestingly, the helix-shaped poly $(1,5-$ NAPD) chains tend to be bundled up during the depositing process and ended as salient nanorods. By modifying the morphology of normal AC electrodes, ions can be efficiently inserted/extracted from the molecular chains, and consequently, the poly(1,5-NAPD)/AC-based ZHSCs presented a remarkable energy density of $195 \mathrm{Wh} \mathrm{kg}^{-1}$ and $91 \%$ capacity retention after 10,000 cycles. Hence, there is no doubt that the polymer-carbon hybrid electrode can tremendously improve the capacity of carbon materials and relatively stabilize the unstable structure of conducting polymer.

To further investigate the inner mechanism of polymer-carbon hybrid electrode, Huang et al. reported a polydopamine-coated porous carbon cloth (PDA@PCC) electrode and the preparation process of PDA@PCC electrode is illustrated in Fig. 6d [154]. Figure 6e presents the Nyquist plots of three different electrodes of CC, PCC and PDA @ PCC electrodes. Typically, PDA@PCC and PCC showed a shorter tail than $\mathrm{CC}$, which represented a better ion diffusion ability based on the hydrophilic property and pore structures. However, due to the defects of PDA polymerization, PDA@ PCC exhibited a relatively larger semicircle in the high-frequency region. The Raman spectra of diverse electrodes are studied in Fig. 6f, the intensity ratio $\left(I_{D} / I_{G}\right)$ of $\mathrm{D}$ band and $\mathrm{G}$ band generally indicates the disorder degree of carbonbased materials. The PDA@PCC hybrid electrodes possess the lowest ratio of 0.93 , which is beneficial for achieving stable electrochemical performance. It is believed that the introduction of PDA can greatly restore the disorder degree from 1.20 to 0.93 and retain the porosity of PCC during the synthesis processes of PDA@PCC. Hence, with the lowest disorder degree, PDA@PCC-based ZHSCs delivered a great capacity retention of $100 \%$ after 10,000 cycles. Moreover, Fig. $6 \mathrm{~g}$ shows the XPS spectra of poly(1,5-NAPD)/AC, poly(1,5-NAPD)/Pt and bare AC samples. It can be proved that the synergetic effect of polymer-carbon hybrid electrode is not simple superposition but proportional integration according to the N $1 \mathrm{~s}$ spectra of each electrode. Simultaneously, the weak $\mathrm{S}$ and $\mathrm{F}$ signals were reported as the binder between the poly(1,5-NAPD) and AC during the electrodepositing process.
2.2.2.3 Transition Metal Compounds (TMCs) TMCs electrode materials such as transition metal oxides (TMOs), nitrides (TMNs), hydroxides and sulfides have been widely investigated in the metal-ion batteries and capacitors on account of their outstanding characteristics like multiple metal valences, high theoretical capacitance, abundant natural source and diversiform synthetic methods [161, 162]. Hydrous $\mathrm{RuO}_{2} \cdot \mathrm{H}_{2} \mathrm{O}$, as the first demonstrated pseudocapacitive materials, have been used as the advanced Cap electrode materials in many ESSs $[163,164]$. As a result, Dong et al. employed amorphous $\mathrm{RuO}_{2} \cdot \mathrm{H}_{2} \mathrm{O}$ as the cathodes of $\mathrm{Zn} / / \mathrm{Cap}$ ZHSCs based on a fast and safe Zn-ion pseudocapacitive storage mechanism [165]. The electrochemical performance of anhydrous $\mathrm{RuO}_{2}$ and hydrous $\mathrm{RuO}_{2} \cdot \mathrm{H}_{2} \mathrm{O}$ were compared in the system of ZHSCs. Although the anhydrous $\mathrm{RuO}_{2}$ possess higher electrical conductivity than hydrous $\mathrm{RuO}_{2} \cdot \mathrm{H}_{2} \mathrm{O}$, the bound $\mathrm{H}_{2} \mathrm{O}$ in $\mathrm{RuO}_{2}$ can facilitate the ions diffusion and thus lead to a better capacity than anhydrous $\mathrm{RuO}_{2}$. According to Dunn's method, 79.0-96.4\% capacitance of $\mathrm{RuO}_{2} \cdot \mathrm{H}_{2} \mathrm{O}$ cathodes originates from the surface-controlled capacitive process. The capacitance is ascribed to the co-existence of EDLC and redox pseudocapacitance, and the majority part of the capacitance is devoted by the pseudocapacitance since the SSA of $\mathrm{RuO}_{2} \cdot \mathrm{H}_{2} \mathrm{O}$ electrodes is only $57 \mathrm{~m}^{2} \mathrm{~g}^{-1}$. Benefiting from the high conductivity $\left(>100 \mathrm{~S} \mathrm{~cm}^{-1}\right)$ and abundant surface redox reactions of hydrous $\mathrm{RuO}_{2} \cdot \mathrm{H}_{2} \mathrm{O}$, the assembled $\mathrm{Zn} / / \mathrm{RuO}_{2} \cdot \mathrm{H}_{2} \mathrm{O}$ ZHSCs enabled ultra-fast $\mathrm{Zn}$-ion storage speed and presented splendid energy density of 119 Wh $\mathrm{kg}^{-1}$ with a voltage window of 0.4-1.6 V. Particularly, it still kept a high energy density of $82 \mathrm{Wh} \mathrm{kg}^{-1}$ under an ultra-high power output of $16,740 \mathrm{~W} \mathrm{~kg}^{-1}$. It is amazing to reach such balanced electrochemical performance while the reported LIBs and ZIBs can only deliver a power density of $1-10 \mathrm{~kW} \mathrm{~kg}{ }^{-1}$. Moreover, the rate performance of the $\mathrm{Zn} / / \mathrm{RuO}_{2} \cdot \mathrm{H}_{2} \mathrm{O}$ ZHSCs is superior than many TMCs and the ZHSCs also presented an outstanding capacity retention of $87.5 \%$ after 10,000 cycles at a current density of $20 \mathrm{~A} \mathrm{~g}^{-1}$.

Titanium nitride ( $\mathrm{TiN}$ ) with superb electrical conductivity $\left(27 \mu \Omega \mathrm{cm}^{-1}\right)$ and chemical/electrochemical stability have also been considered as a promising pseudocapacitive electrode material for energy storage [166-168]. Zhi group employed the TiN electrode as the cathode materials for $\mathrm{Zn} / / \mathrm{Cap} \mathrm{ZHSCs}$, and coupled with $\mathrm{Zn}$ metal anode to form $\mathrm{Zn} / / \mathrm{TiN}$ ZHSCs [169]. Although TiN is a pseudocapacitive material, no evident redox peaks of TiN electrodes are detected in the $\mathrm{CV}$ curves during charging/discharging process, indicating the predominant Cap behaviors of the TiN cathode materials in the system of ZHSCs. Owing to 
the excellent conductivity and splendid stability of TiN, the $\mathrm{Zn} / /$ TiN ZHSCs delivered an ultra-high capacity of $489.8 \mathrm{~F}$ $\mathrm{g}^{-1}$ at $0.2 \mathrm{~A} \mathrm{~g}^{-1}$ in the $\mathrm{ZnSO}_{4}$ electrolyte. In addition, the self-discharge rate of TiN-based ZHSCs is extremely low due to the high stability of TiN-SO $\mathrm{S}_{4}$ structure after adsorption process, which remained $83.9 \%$ of initial capacity after $500 \mathrm{~h}$ resting time.

Herein, it is believed that the TMCs materials with diverse properties and $\mathrm{Zn}$-ion storage mechanisms are competitive pseudocapacitive electrodes for high-performance ZHSCs. However, we need to draw a distinction between the pseudocapacitive TMCs and the batterytype TMCs like manganese oxides and vanadium oxides, as well as other reported ZBC. Compared with the Cap TMCs electrodes, the redox process of battery-type TMCs is responsible for the phase transition of electrode materials [142]. Additionally, the potential of the battery-type electrodes will remain approximately constant, presenting a distinct charging/discharging platform in the GCD curves, which agrees with the phase rule and follows the Nernst equation. Correspondingly, a couple of welldefined redox peaks can be detected in the $\mathrm{CV}$ curves. Hence, the pseudocapacitive TMCs electrodes performed as the Cap cathode in the Zn//Cap ZHSCs while the battery-type TMOs acted as the $\mathrm{ZBC}$ in the Cap//ZBC ZHSCs.

In summary, Cap electrode materials including HSSA carbon electrodes, heteroatom-doped carbon electrodes, graphene-based electrodes and pseudocapacitive electrodes (MXenes, polymer-carbon hybrid electrodes and TMCs) are the most investigated materials among all candidate electrodes for ZHSCs. HSSA carbon electrodes can provide sufficient EDLC and power density for ZHSCs, however, the EDLC is not enough to offer balanced energy density which should match with battery-type electrodes in ZHSCs. Graphene-based electrodes possess excellent properties of ultra-high intrinsic capacitance $\left(\sim 21 \mu \mathrm{F} \mathrm{cm}^{-2}\right)$, high theoretical SSA of $2630 \mathrm{~m}^{2} \mathrm{~g}^{-1}$, ultra-low RC constants $(<200 \mu \mathrm{s})$ and high chemical stability. Therefore, graphenebased ZHSCs present better longevity than other ZHSCs. Heteroatom-doped carbon electrodes and polymer-carbon hybrid electrodes are both based on the fundamental carbon electrodes, equipping the carbon electrodes with abundant chemical pseudocapacitive reactions, which can effectively enhance the energy density of normal carbon electrodes. As for polymer-carbon hybrid electrodes, although the energy density of carbon electrodes is promoted, the conducting polymer materials show poor cycling performance on account of the poor stability, active material loss and over-oxidative degradation. MXenes show balanced electrochemical performance but suffer from the low operating voltage window and short longevity. Notably, owing to the superb electrical conductivity and considerable pseudocapacitance, TMC-based ZHSCs achieve large capacity and fast charging/discharging rate. Therefore, among these reported Cap electrodes, graphene-based electrodes, heteroatom-doped carbon electrodes and TMCs electrodes are considered as the most promising Cap electrodes for the practical application of ZHSCs, and it is believed that more and more excellent Cap electrodes can be discovered as the fast development of ZHSCs. When designing an appropriate Cap electrode for ZHSCs with perfect performance, it is significant to keep the balance between power density, energy density and stability, which can be further attributed to the balance between EDLC and chemical pseudocapacitive reactions.

As overviewed above and summarized in Table 1, the $\mathrm{Zn} / /$ Cap ZHSCs with different kinds of electrode materials have been expatiated and further concluded. More importantly, the intricate and unique storage mechanisms of Cap electrodes are carefully summarized as several parts, where the adsorption/desorption processes based on the EDLC are always effective while the redox reaction or insertion/desertion processes may exist depending on the diverse strategy of Cap cathodes. Based on the well-designed $\mathrm{Zn}$ anodes and various kinds of Cap electrodes, reported $\mathrm{Zn} / / \mathrm{Cap} \mathrm{ZHSCs}$ showed ultra-long cycling lifetime and excellent integration of high energy density and power density. The Zn//Cap ZHSCs can reach the approximate energy density of LIBs but higher power density, making the ZHCSs a full-scale ESS for wild applications such as wearable electronics, electric vehicles, large-scale facilities and so on. On account of the splendid rate property, ZHSCs can easily change service conditions as the practical current density demands change. However, the voltage window of $\mathrm{Zn} / / \mathrm{Cap}$ ZHSCs is still too low (lower than $1.8 \mathrm{~V}$ ) to fulfill the demand of the practical applications. On the contrary, the Cap//ZBC ZHSCs can readily reach $2.0 \mathrm{~V}$ in the normal $\mathrm{ZnSO}_{4}$ electrolyte. By far, the reports about Cap//ZBC ZHSCs are few, thus, it is highly recommended to make some explorations on the substantial foundation of various ZBC which have been widely applied in the system of ZIBs. 


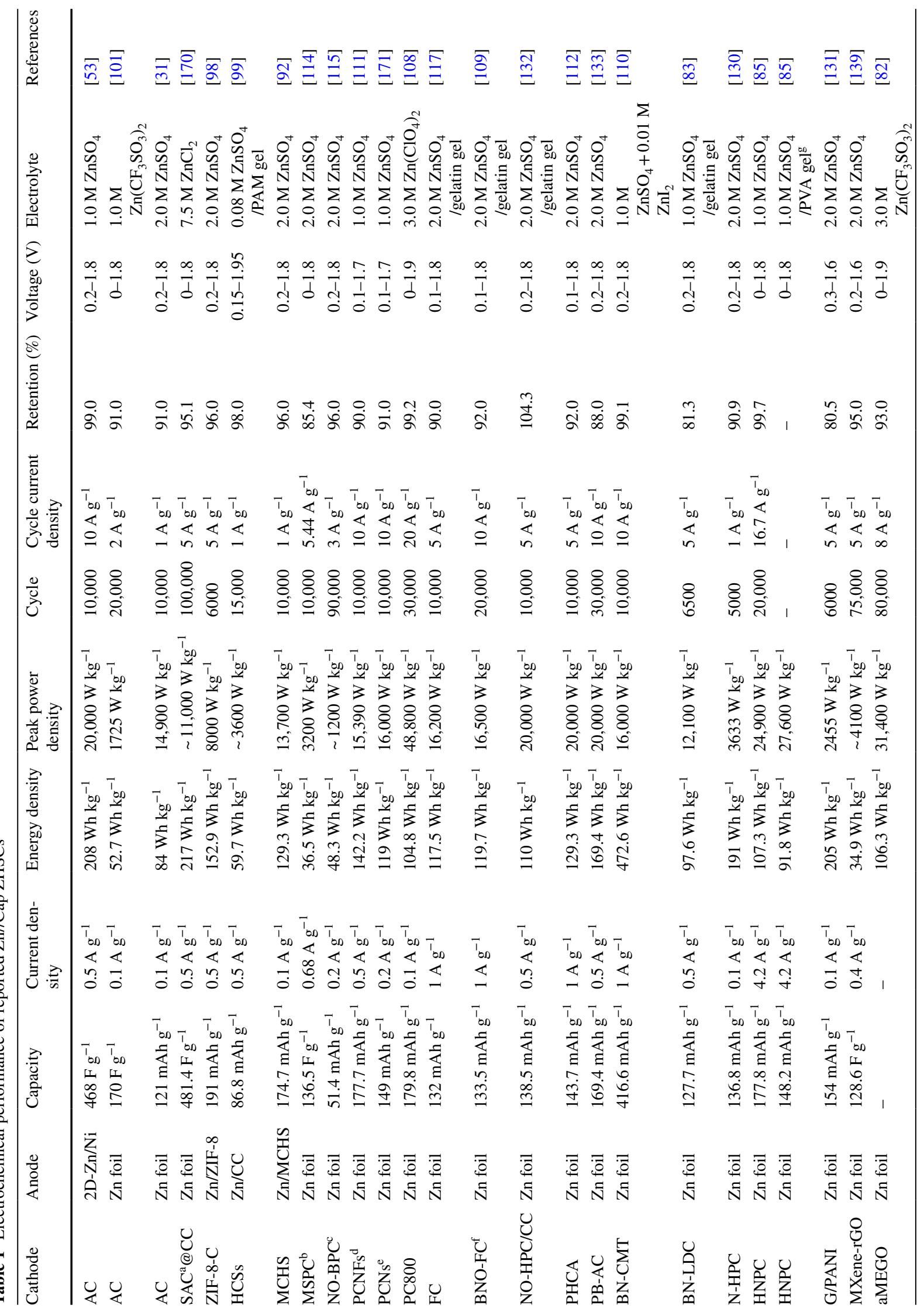




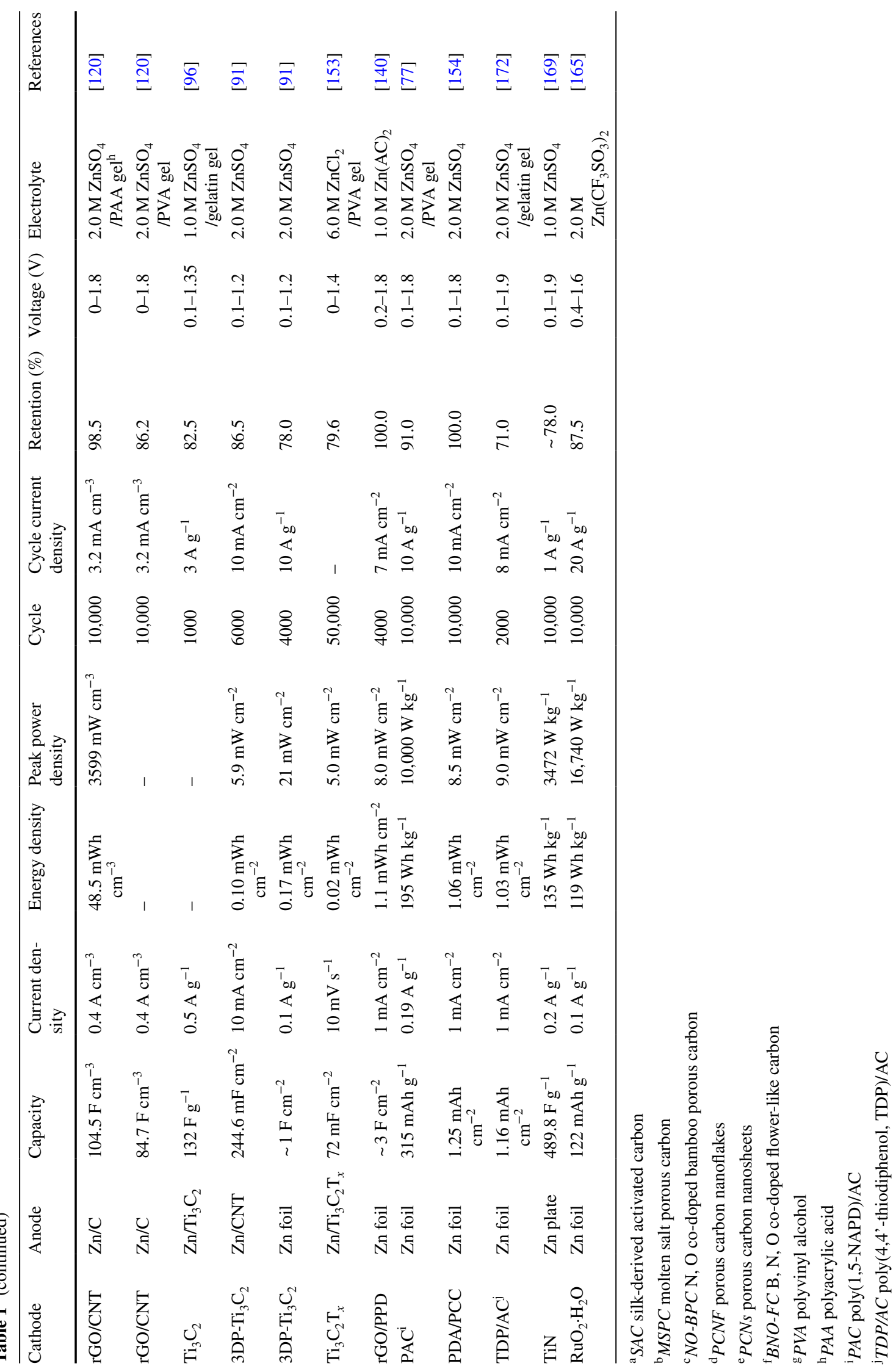




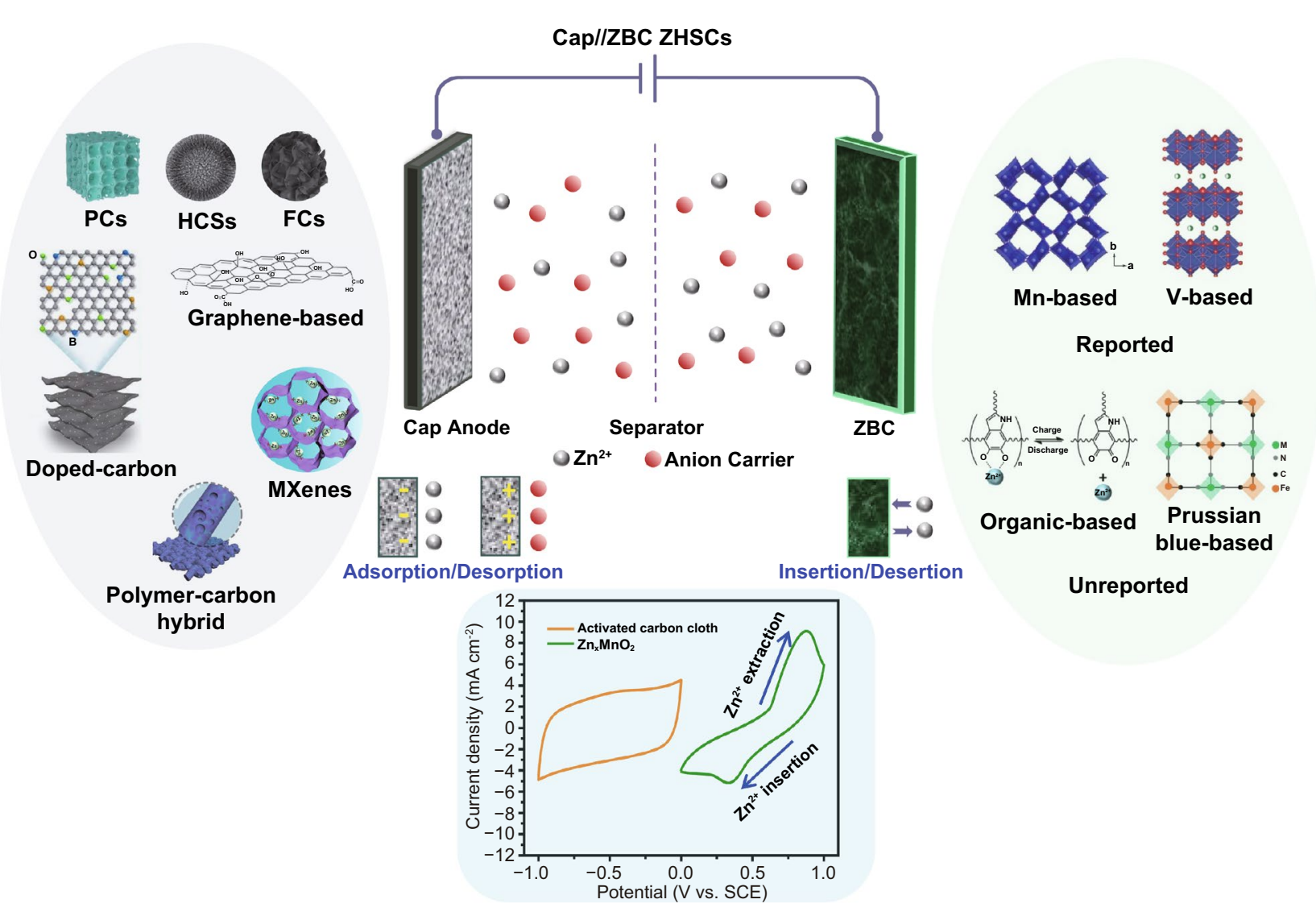

Fig. 7 Schematic illustration (Cap anodes, ZBC, energy storage mechanisms and CV behaviors) of Cap//ZBC ZHSCs

\subsection{Cap//ZBC ZHSCs}

Generally, the ZHSCs are constructed by the integration of ZIBs and supercapacitors, where the Cap electrodes are typically carbon and pseudocapacitive electrodes as we reviewed in Sect. 2.2, the battery-type electrodes are ZBC and $\mathrm{Zn}$ anodes in the ZIB systems. Before this section, we amply discussed the Zn//Cap ZHSCs, which comprise Zn anodes and Cap cathodes. Conversely, the design theory of Cap//ZBC ZHSCs is to replace the $\mathrm{Zn}$ anode in ZIBs with Cap electrodes. Hence, different from the $\mathrm{Zn} / / \mathrm{Cap}$ ZHSCs, the Cap electrodes will act as the anode and Znion battery-type materials will act as the cathode in the Cap//ZBC ZHSCs. Notably, many potential ZBC of ZHSCs have been reported in the ZIBs, such as manganese-based oxide, vanadium-based oxides, prussian blue and analogues, spinels, etc.

As illustrated in Fig. 7, the Cap anodes, ZBC, energy storage mechanism and $\mathrm{CV}$ behavior of Cap//ZBC ZHSCs are exhibited. Same as the Zn//Cap ZHSCs, the electrolytes are Zn salt solutions. Basically, Cap electrodes perform the same energy storage mechanism in the $\mathrm{Zn} / / \mathrm{Cap}$ ZHSCs, but act as anode opposed to the ZBC. The CV curve of ACC anodes in Fig. 7 presented a typical rectangular shape of Cap anodes. On the contrary, the CV plot of $\mathrm{Zn}_{x} \mathrm{MnO}_{2}$ cathodes showed two obvious redox peaks at approximately $0.33 \mathrm{~V} / 0.88 \mathrm{~V}$ ( $v s$. SCE), corresponding to the insertion/desertion reaction processes of ZBC. The $\mathrm{Zn}$-ion insertions happen during the discharging processes of ZHSCs, while the Zn-ion desertions occur during the charging periods. Gratifyingly, many ZBC can reversibly proceed insertion/extraction reactions of $\mathrm{Zn}$-ions, which have been widely confirmed in ZIBs, including manganese oxides $\left(\mathrm{MnO}_{2}\right.$ with a different crystalline structure, $\mathrm{Mn}_{2} \mathrm{O}_{3}$, etc.) [87, 173], vanadium oxides $\left(\mathrm{V}_{2} \mathrm{O}_{5}, \mathrm{~V}_{2} \mathrm{O}_{7}, \mathrm{~V}_{3} \mathrm{O}_{8}\right.$, and homologous derivatives, etc.) [174-177], prussian blue and analogues $[8,178-180]$ and spinels $\left(\mathrm{ZnMn}_{2} \mathrm{O}_{4}\right.$, etc. $)$ [181-184]. Although the ionic radius of $\mathrm{Zn}$-ion is small 
$(0.75 \AA)$, the hydrated ionic radius of $\mathrm{Zn}$-ion $(3.40-$ $3.82 \AA$ ) raises strong demand for suitable insertion cathode materials of ZHSCs [87]. Some special crystal structures like layered structure $\left(\delta-\mathrm{MnO}_{2}, \mathrm{~V}\right.$-based materials, etc.), tunnel structure $\left(\alpha-\mathrm{MnO}_{2}, \gamma-\mathrm{MnO}_{2}\right.$, etc.) and framework (prussian blue and analogues, spinels, etc.) can effectively store $\mathrm{Zn}$-ion via reversible insertion/desertion processes. A large and stable crystal structure can store a great number of $\mathrm{Zn}$-ion and then provide considerable capacity by reversible chemical redox reactions between $\mathrm{Zn}$-ion and cathode materials.

When the ZHSCs are assembled based on Cap anodes and ZBC, the Cap//ZBC ZHSCs can provide a higher voltage window of $0-2.0 \mathrm{~V}$ and more possibilities like Zn-dendrite-free ZHSCs. However, only a few ZBC (e.g., manganese-based oxides and vanadium-based oxides) have been applied in this configuration of ZHSCs and many problems still exist. Here, we will summarize some reported studies of Cap//ZBC ZHSCs in the following review, trying to draw more attention to the $\mathrm{ZBC}$ and the second electrode configuration of Cap//ZBC ZHSCs.
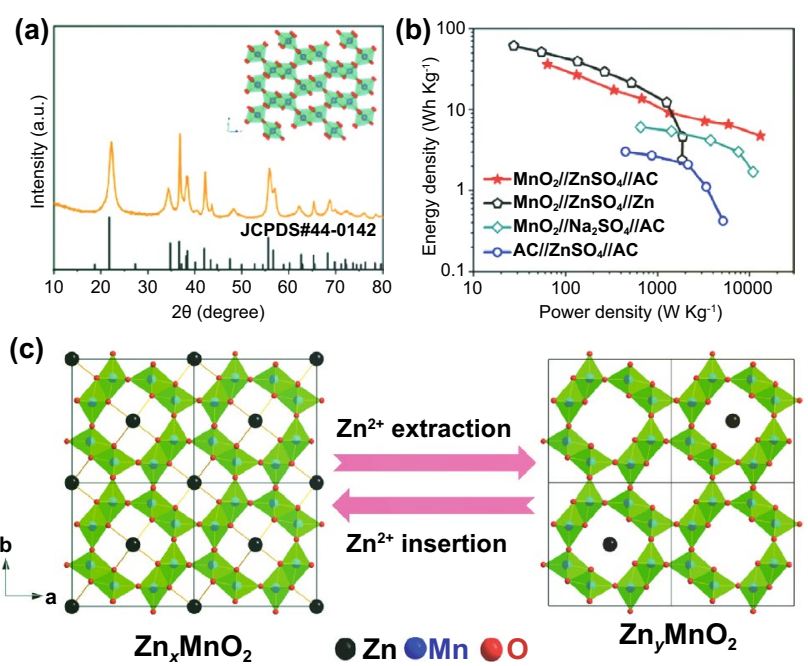

\subsubsection{Manganese-Based Oxides}

Manganese oxides, with many outstanding properties like low cost, high theoretical capacity, tunable and multifarious crystal structure, etc., have been well investigated in various ESSs [185-187]. Among various manganese oxides, $\mathrm{MnO}_{2}$ is one of the most promising manganese oxides. By linking the fundamental units $\mathrm{MnO}_{6}$ octahedra with shared edges and vertices, tunnel-type $\alpha-\mathrm{MnO}_{2}, \beta-\mathrm{MnO}_{2}, \delta-\mathrm{MnO}_{2}$, $\varepsilon-\mathrm{MnO}_{2}$ and layered $\gamma-\mathrm{MnO}_{2}$ are obtained [87]. Generally, many $\mathrm{MnO}_{2}$ cathodes with diverse crystal structures have been employed in ZIBs while only a few were reported in ZHSCs.

As the pioneer of the Cap//ZBC ZHSCs, Kang group reported the $\mathrm{AC} / / \gamma-\mathrm{MnO}_{2} \mathrm{ZHSCs}$ with a wide voltage window of $0-2.0 \mathrm{~V}$, the as-assembled ZHSCs showed a capacity of $54.1 \mathrm{mAh} \mathrm{g}^{-1}$ in $2.0 \mathrm{M} \mathrm{ZnSO}_{4}$ electrolyte while exhibited a capacity of $83.8 \mathrm{mAh} \mathrm{g}^{-1}$ in the $2.0 \mathrm{M} \mathrm{ZnSO}_{4}$ and $0.5 \mathrm{M} \mathrm{MnSO}_{4}$ electrolyte [188]. The XRD pattern and tunnel crystal structure of $\gamma-\mathrm{MnO}_{2}$ cathode are displayed in Fig. 8a, the insertion/desertion processes of $\mathrm{Zn}$-ion occur in the tunnel structure of $\gamma-\mathrm{MnO}_{2}$ cathodes. However, due to
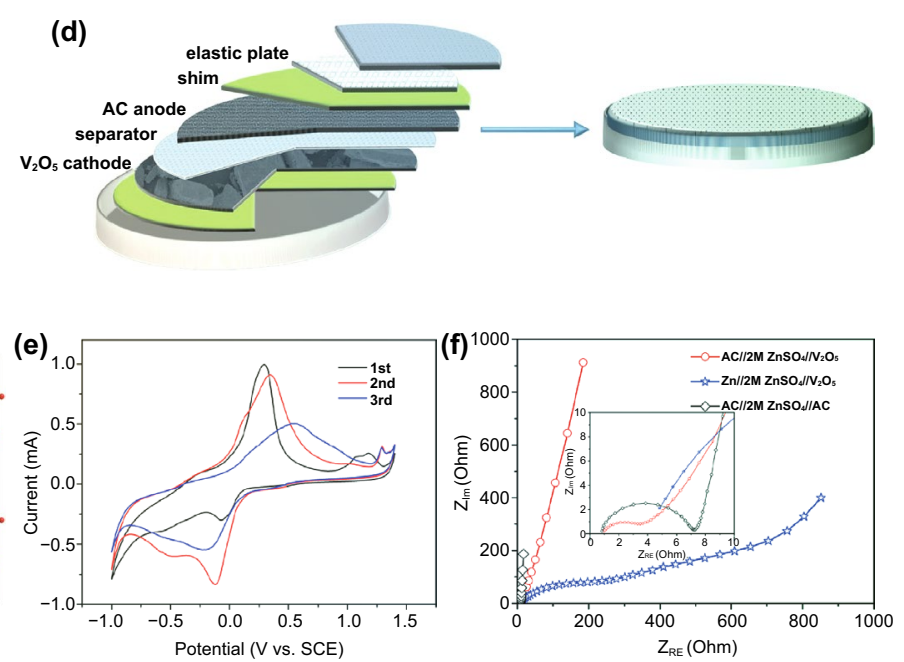

Fig. 8 a XRD pattern and crystal structure (inset) of $\gamma-\mathrm{MnO}_{2}$ cathodes. b Ragone plots of various $\gamma$-MnO $\mathrm{M}_{2}$-based ESSs. Reproduced with permission from Ref. [188]. Copyright 2019, Elsevier. c Schematic illustration of the $\mathrm{Zn}$-ion storage mechanisms in the tunnel-structure $\mathrm{Zn}_{x} \mathrm{MnO}_{2}$ cathode. Reproduced with permission from Ref. [34]. Copyright 2020, Wiley-VCH. d Full cell schematic of AC//2.0 M ZnSO $\mathrm{M}_{4} / / \mathrm{V}_{2} \mathrm{O}_{5} \mathrm{ZHSCs}$ e $\mathrm{CV}$ curves tested by three-electrode system of $\mathrm{V}_{2} \mathrm{O}_{5}$. f Nyquist plots of relevant devices, including ZHSC, ZIB and supercapacitor. Reproduced with permission from Ref. [189]. Copyright 2019, Springer Nature

the solubility and poor electrical conductivity of Mn-based materials, the $\mathrm{AC} / / \gamma-\mathrm{MnO}_{2}$ ZHSCs can merely keep $65.3 \%$ 
of initial capacity after 3000 cycles without the $\mathrm{MnSO}_{4}$ additive electrolyte. When changing the electrolyte from $\mathrm{ZnSO}_{4}$ to $\mathrm{Zn}\left(\mathrm{CF}_{3} \mathrm{SO}_{3}\right)_{2}$, the cycling performance of $\mathrm{AC} / / \gamma-\mathrm{MnO}_{2}$ ZHSCs can be tremendously improved to $93.4 \%$ after longer 5000 cycles. Generally, the $\mathrm{CF}_{3} \mathrm{SO}_{3}{ }^{-}$anion results in lower charge transfer resistance and higher ion diffusion rate than $\mathrm{SO}_{4}{ }^{2-}$ anion. Furthermore, the by-products are not visibly detected in the $\mathrm{Zn}\left(\mathrm{CF}_{3} \mathrm{SO}_{3}\right)_{2}$ electrolyte due to the reduced interaction between $\mathrm{Zn}$-ions and adjacent water molecules [82]. When compared with the $\mathrm{Zn} / / \gamma-\mathrm{MnO}_{2} \mathrm{ZIBs}$ (Fig. 8b), the $\mathrm{AC} / / \gamma-\mathrm{MnO}_{2} \mathrm{ZHSCs}$ presented a higher power density of $13,000 \mathrm{~W} \mathrm{~kg}{ }^{-1}$ and the poor rate performance of $\gamma-\mathrm{MnO}_{2}-$ based ZIBs is greatly enhanced in the fast ZHSCs. Although the capacity of $\mathrm{AC} / / \gamma-\mathrm{MnO}_{2} \mathrm{ZHSCs}$ is still unsatisfactory, the strengths of Cap//ZBC ZHSCs like larger voltage window, high power density, excellent rate performance and unique energy storage mechanisms are presented.

In addition, Mai group recently investigated the application of tunnel-type $\alpha-\mathrm{MnO}_{2}\left(\mathrm{Zn}_{x} \mathrm{MnO}_{2}\right)$ and layered $\delta$ $\mathrm{MnO}_{2}$ cathodes in ZHSCs, the active carbon cloth (ACC)// $\mathrm{Zn}_{x} \mathrm{MnO}_{2}$ and $\mathrm{ACC} / / \delta-\mathrm{MnO}_{2} \mathrm{ZHSCs}$ are prepared and tested [34]. As depicted in Fig. 8c, some pre-intercalation $\mathrm{Zn}$-ions are stored in the tunnel structure of $\mathrm{Zn}_{x} \mathrm{MnO}_{2}$ cathode before electrochemical tests. Additionally, the Zn-ions are extracted from the tunnel structure when the ZHSCs are charging and conversely, inserted into the tunnel structure during discharging periods. The capacity of $\mathrm{MnO}_{2}$ cathodes may fluctuate a lot at first few cycles since a great number of $\mathrm{Zn}$-ions are left in the tunnel structure, which leads to the unbalanced $\mathrm{Zn}$-ion insertion/desertion reactions. However, depend on the pre-intercalation $\mathrm{Zn}$-ion in the tunnel structure, the $\mathrm{Zn}_{x} \mathrm{MnO}_{2}$ cathode is stabilized in advance and then performed steady cycling performance. Compared with the tunnel structure of $\gamma-\mathrm{MnO}_{2}(1 \times 1$ and $1 \times 2$ tunnel structure: $2.3 \times 2.3 \AA$ and $2.3 \times 4.6 \AA)$, the $\alpha-\mathrm{MnO}_{2}(2 \times 2$ tunnel structure: $4.6 \times 4.6 \AA$ ) possess a larger and more suitable tunnel structure for $\mathrm{Zn}$-ions storage due to the close hydrated ionic radii of $\mathrm{Zn}$-ions (4.3 $\AA$ ). Accordingly, the $\mathrm{ACC} / / \mathrm{Zn}_{x} \mathrm{MnO}_{2}$ ZHSCs remained $83.1 \%$ of initial capacity after 5000 cycles, and the ex-situ XRD spectra of $\mathrm{Zn}_{x} \mathrm{MnO}_{2}$ cathode presented no evident excursion during the charging/discharging processes. Layered $\delta$ - $\mathrm{MnO}_{2}$ is also a promising $\mathrm{Zn}$-ion storage cathode with an ultra-large interlayer spacing of $\sim 7 \AA$. Nevertheless, the structure of $\delta-\mathrm{MnO}_{2}$ is prone to collapse in sulfate solution during the insertion/desertion processes of $\mathrm{Zn}$-ions, thus, the capacity retention of $\mathrm{ACC} / / \delta-\mathrm{MnO}_{2}$ ZHSCs quickly fade to $83.2 \%$ after 10 cycles and then drop to $70.4 \%$ after 35 cycles, finally end at $45.3 \%$ after 5000 cycles. Hence, it is believed that the $\mathrm{Zn}$-ion pre-intercalation can be a way to promote the stability of manganesebased cathodes. The $\mathrm{ACC} / / \mathrm{Zn}_{x} \mathrm{MnO}_{2} \mathrm{ZHSCs}$ delivered an ultra-high and stable areal capacity of $1745.8 \mathrm{mF} \mathrm{cm}^{-2}$ at $2 \mathrm{~mA} \mathrm{~cm}{ }^{-2}$. Meanwhile, ACC//Zn $\mathrm{AnO}_{2} \mathrm{ZHSCs}$ performed a wide operating voltage window of 0-2.0 V and excellent areal power density of $20.1 \mathrm{~mW} \mathrm{~cm}^{-2}$, which is vital for many flexible and wearable electronic devices.

Notably, a novel ZHSC of $\mathrm{Ti}_{3} \mathrm{C}_{2} \mathrm{~T}_{x} / / \mathrm{MnO}_{2}$-CNTs was proposed by Wang et al., the pseudocapacitive MXene materials act as the Cap anode and the battery-type $\mathrm{MnO}_{2}-\mathrm{CNT}$ act as the cathode [190]. The electrode properties were demonstrated by the three-electrode system, where the $\mathrm{MnO}_{2}-\mathrm{CNTs}$ presented typical charge/discharge platforms in the GCD curves and a couple of redox peaks were detected in the $\mathrm{CV}$ curves. On the contrary, the $\mathrm{Ti}_{3} \mathrm{C}_{2} \mathrm{~T}_{x}$ anode showed distinct Cap behaviors with straight up and down GCD curves. These results confirmed that the $\mathrm{Zn}$-ion storage mechanism of $\mathrm{ZBC}$ is pretty different from the pseudocapacitive electrode. The pseudocapacitive TMCs should not be confused with TMCs ZBC. Benefit from the balanced electrode configuration of Cap//ZBC ZHSCs and excellent electrochemical performance of $\mathrm{MnO}_{2}$, the $\mathrm{Ti}_{3} \mathrm{C}_{2} \mathrm{~T}_{x} / / \mathrm{MnO}_{2}-\mathrm{CNTs}$ ZHSCs exhibited splendid capacity of $115.1 \mathrm{~F} \mathrm{~g}^{-1}$ as well as remarkable longevity of $83.6 \%$ capacity retention after 15,000 cycles, which is the best cycling performance in the system of Cap//ZBC ZHSCs.

For many manganese-based oxide cathodes, the crystal transformations will occur during the insertion/desertion processes of different cations or water molecules, and different structures of manganese-based oxide cathodes may lead to different Zn-ion storage performance in ZHSCs. By far, the $\alpha-\mathrm{MnO}_{2}$ exhibited a prominent electrochemical performance as well as stable tunnel structure among various manganese-based oxide cathodes. Although numerous advantages the manganese-based oxide cathodes have shown, the poor rate capability, easy-dissolution in electrolyte and relatively unstable structure are still challenges in the application of Cap//manganese oxide ZHSCs. Hence, more efforts should be devoted to investigating suitable manganese oxide cathodes for high-performance ZHSCs and create more possibility for Cap//ZBC ZHSCs. 


\subsubsection{Vanadium-Based Oxides}

Apart from the manganese-based oxides, vanadium-based oxides are another widely applied ZBC for ZHSCs. With high theoretical capacity, low cost and stable layer crystal structure, vanadium-based oxide cathodes are suitable for multivalent ions storage [191, 192]. However, by far, only one research has been reported about the ZHSCs based on the vanadium-based oxide cathodes [189]. Therefore, in this section, the rare vanadium-based ZHSC will be discussed in detail.

As illustrated in Fig. 8d, Ma et al. employed $\mathrm{V}_{2} \mathrm{O}_{5}$ materials as the cathode of ZHSCs and assembled the $\mathrm{AC} / / \mathrm{V}_{2} \mathrm{O}_{5}$ ZHSCs where the $\mathrm{V}_{2} \mathrm{O}_{5}$ materials and $\mathrm{AC}$ powder act as the cathode and anode, respectively [189]. Typically, $\mathrm{V}_{2} \mathrm{O}_{5}$ cathodes tend to proceed an activated process before longtime charging/discharging according to the $\mathrm{CV}$ curves shown in Fig. 8e, where the redox peaks of $\mathrm{V}_{2} \mathrm{O}_{5}$ cathode sharply changed at the first three cycles. It is believed that the incipient charging/discharging process of $\mathrm{V}_{2} \mathrm{O}_{5}$ cathode leads to structural transformations, and the $\mathrm{V}_{2} \mathrm{O}_{5}$ cathodes turn out to be stable after activation. The possible electrochemical reaction is as the following Eq. (2-2):

$\mathrm{V}_{2} \mathrm{O}_{5}+x \mathrm{Zn}^{2+} \leftrightarrow \mathrm{Zn}_{x} \mathrm{~V}_{2} \mathrm{O}_{5}(x \approx 0.3)$

Similarly, the $\mathrm{AC} / / \mathrm{V}_{2} \mathrm{O}_{5}$ ZHSCs can also achieve a wide voltage window of $0-2.0 \mathrm{~V}$, which is unreachable for vanadium-based ZIBs (most $<1.4 \mathrm{~V}$ ). Furthermore, based on the EIS results of various ESSs (Fig. 8f), the $\mathrm{AC} / / \mathrm{V}_{2} \mathrm{O}_{5}$ ZHSCs showed the smaller charge transfer resistance and higher ion diffusion rate than $\mathrm{Zn} / / \mathrm{V}_{2} \mathrm{O}_{5}$ ZIBs and normal AC//AC supercapacitors. Thus, ZHSCs present superiority in comparison with ZIBs and supercapacitors. However, the $\mathrm{AC} / / \mathrm{V}_{2} \mathrm{O}_{5}$ ZHSCs only provided a limited capacity of 57.4 $\mathrm{mAh} \mathrm{g}{ }^{-1}$ and an energy density of $34.6 \mathrm{Wh} \mathrm{kg}^{-1}$. Also, the ZHSCs cannot maintain appropriate coulombic efficiency at an ultra-low current density of $0.1 \mathrm{~A} \mathrm{~g} \mathrm{~g}^{-1}$, which can be attributed to the polarization of $\mathrm{V}_{2} \mathrm{O}_{5}$ at $1.8-2.0 \mathrm{~V}$. Gratifyingly, the cycling stability test of $\mathrm{AC} / / \mathrm{V}_{2} \mathrm{O}_{5}$ ZHSCs is quite surprised, which presented a capacity retention of 97.3\% after 6000 cycles at relatively low cycling current density of $0.5 \mathrm{~A} \mathrm{~g}^{-1}$, indicating that the $\mathrm{V}_{2} \mathrm{O}_{5}$ cathodes possess a suitable and stable structure for ZHSCs. As we all know, the excellent performance of Cap//ZBC ZHSCs is generally determined by the ZBC used. However, only one work about vanadium-based cathode of ZHSCs has been reported, moreover, the $\mathrm{Zn}$-ions storage mechanism of vanadium-based ZHSCs is still ambiguous and the capacity of vanadium-based ZHSCs is unsatisfied. Therefore, more efforts should be devoted to promoting the development of vanadium-based oxide cathodes for high-performance ZHSCs.

As summarized in Table 2, the Cap//ZBC ZHSCs presented prominent characteristics like high voltage window, abundant electrode collocations and special properties depend on various ZBC. More significantly, the Zn-foil-free ZHSCs can avoid the potential safety hazard of $\mathrm{Zn}$ dendrite, leading to a $\mathrm{Zn}$-dendrite-free ZHSC. Although the electrochemical performance of Cap//ZBC ZHSCs is not enough for practical applications, we believe that after sufficient investigations and creations, the Cap//ZBC ZHSCs will perform splendid electrochemical performance which may even superior to the Zn//Cap ZHSCs. To sum up, the Cap//ZBC ZHSCs still have many difficulties that need to conquer, here we summarize as the following aspects: (1) relatively low energy density, (2) inferior rate performance and (3) weak cycling stability, particularly the $\mathrm{MnO}_{2}$ cathodes which are easy to dissolve. Nevertheless, the in-depth understandings of energy storage mechanisms of various ZBC are plenarily needed, where the mechanisms and extent of reactions may differ from the conditions in ZIBs. The design theory of ZBC for high-performance ZHSCs should be high porosity, HSSA, stable crystal structure $\left(\mathrm{Zn}^{2+}\right.$-adapted) and excellent electrical conductivity. By designing ZBC in a way of the Cap electrode may greatly enhance the rate performance and cycling performance of the Cap//ZBC ZHSCs. Momentously, it is strongly believed that with more attempts and investigations, the electrochemical performance of Cap//ZBC ZHSCs will be enhanced and then become a promising candidate for next-generation ESSs.

\section{Energy Storage Mechanisms of Cap Electrodes}

Importantly, Cap electrodes perform a vital role in both two configurations of ZHSCs, however, the energy storage mechanisms of Cap electrodes are pretty intricate according to the reported ZHSCs [31, 188, 193]. Besides the simple EDLC of the physical adsorption/desorption processes of cations and anions, more results are proved to confirm extra energy 


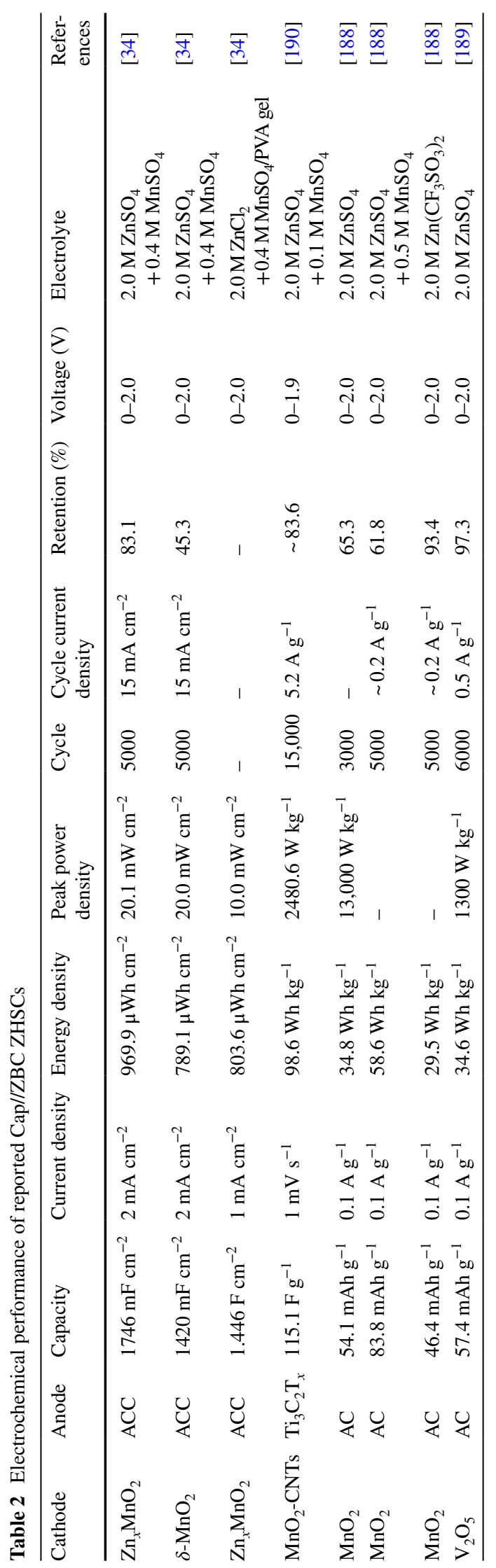


storage mechanisms of Cap electrodes, including precipitation/dissolution and chemical pseudocapacitive reactions. Hence, the related investigations of Cap electrodes will be concluded in the following section [31, 85, 188]. As the fundamental energy storage mechanism, the physical adsorption/desorption processes are presented as following Eqs. (3-1, 3-2), where the $\mathrm{X}^{-}$represents the monovalent anion $\left(\mathrm{Cl}^{-}, \mathrm{NO}_{3}{ }^{-}, \mathrm{CF}_{3} \mathrm{SO}_{3}{ }^{-}\right.$and $\left.\mathrm{CH}_{3} \mathrm{COO}^{-}\right)$, the $\mathrm{Y}^{2-}$ represents the divalent anion $\left(\mathrm{SO}_{4}{ }^{2-}\right)$ [132]:

$$
\begin{aligned}
& \mathrm{C}+\mathrm{Zn}^{2+} / \mathrm{H}^{+} \leftrightarrow \mathrm{C} \| \mathrm{Zn}^{2+} / \mathrm{H}^{+} \\
& \mathrm{C}+\mathrm{X}^{-} / \mathrm{Y}^{2-} \leftrightarrow \mathrm{C} \| \mathrm{X}^{-} / \mathrm{Y}^{2-}
\end{aligned}
$$

\subsection{Precipitation/Dissolution of By-Products}

Typically, the precipitation/dissolution processes of by-products like $\mathrm{Zn}_{4} \mathrm{SO}_{4}(\mathrm{OH})_{6} \cdot \mathrm{nH}_{2} \mathrm{O}$ have been found in the system of ZIBs, especially in the Mn-based and V-based ZIBs [87]. The by-products with loose and porous layers will randomly form on the surface of bare $\mathrm{Zn}$ foil during charging/discharging processes, which leads to the uneven surface and $\mathrm{Zn}$ dendrite, then the poor cycling performance of ZIBs [194, 195]. Therefore, the investigation of formation mechanisms of the by-products in ZHSCs is more necessary since the $\mathrm{Zn}$ anode of ZHSCs can be greatly consumed by the fast charging/discharging processes. Furthermore, the formation of by-products also happened on the Cap electrodes of
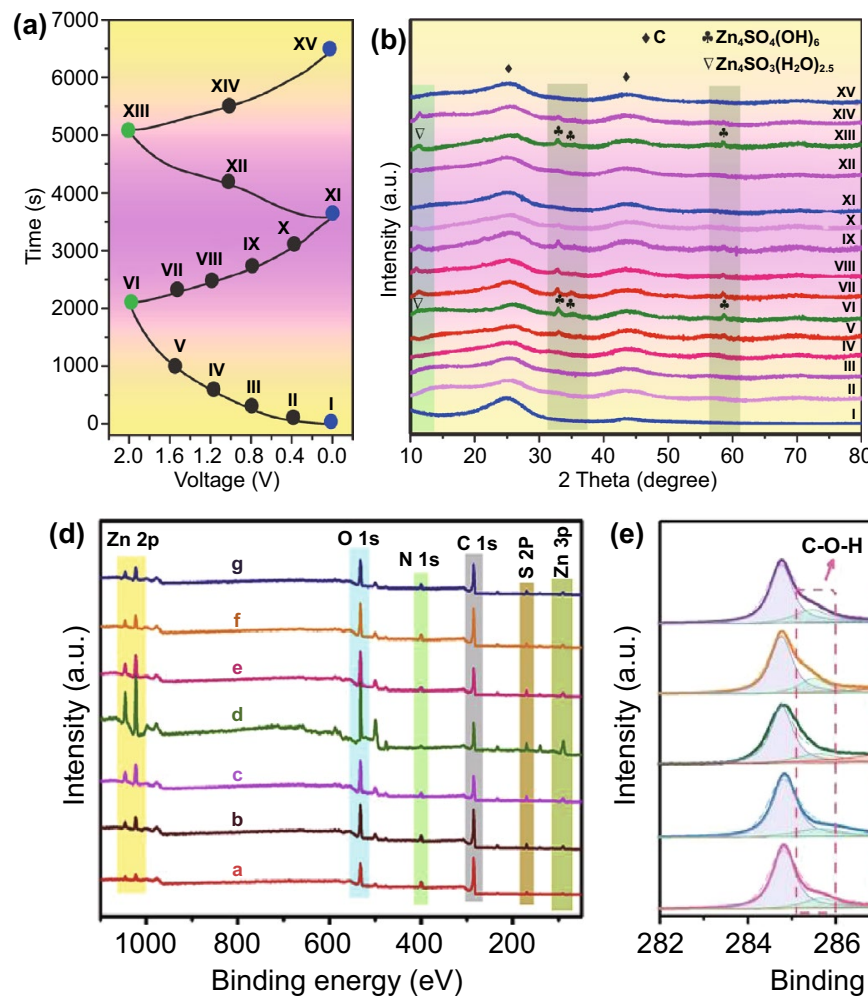

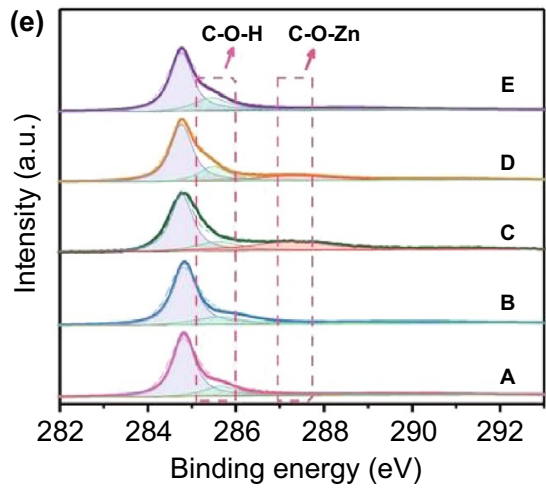

Fig. 9 a GCD profiles of $\mathrm{ACC} / / \mathrm{Zn}_{x} \mathrm{MnO}_{2} \mathrm{ZHSCs}$ at $2 \mathrm{~mA} \mathrm{~cm}{ }^{-2}$. b Corresponding ex-situ XRD patterns and $\mathbf{c}$ SEM images of ACC anodes at different charge/discharge states. Reproduced with permission from Ref. [34]. Copyright 2020, Wiley-VCH. d Ex-situ XPS spectra of carbon cathodes at various states, discharging from a to d and charging from $\mathbf{d}$ to $\mathbf{g}$. Reproduced with permission from Ref. [77]. Copyright 2020, Elsevier. e Ex-situ C $1 \mathrm{~s}$ XPS spectra of HNPC cathodes, discharging from A to C and charging from C to E. f C $1 \mathrm{~s}$ XPS spectra of PC and HNPC cathodes at 0 V. Reproduced with permission from Ref. [85]. Copyright 2019, Wiley-VCH 
ZHSCs, thus, the dual formation of by-products may affect the capacity and cycling performance of ZHSCs during charging/discharging processes.

In the ZHSCs systems, Kang group demonstrated the existence of by-products of $\mathrm{Zn}_{4} \mathrm{SO}_{4}(\mathrm{OH})_{6} \cdot 5 \mathrm{H}_{2} \mathrm{O}$ on both $\mathrm{AC}$ cathode and $\mathrm{Zn}$ anode in $\mathrm{ZnSO}_{4}$ electrolyte after discharging processes (from 1.8 to $0.2 \mathrm{~V}$ ) [31]. Notably, part of by-products is left on the surface of AC cathode after 10 cycles, indicating the irreversible reactions of precipitation/dissolution. Furthermore, the formation of by-products is also detected in other ZHSCs and even in the Cap// ZBC ZHSCs. Particularly, Mai group reported that the byproducts of $\mathrm{Zn}_{4} \mathrm{SO}_{4}(\mathrm{OH})_{6} \cdot \mathrm{nH}_{2} \mathrm{O}$ and $\mathrm{ZnSO}_{3} \cdot 2.5\left(\mathrm{H}_{2} \mathrm{O}\right)$ were also observed on the surface of ACC in $\mathrm{ACC} / / \mathrm{Zn}_{x} \mathrm{MnO}_{2}$ ZHSCs [34]. The ex-situ XRD spectra based on ACC// $\mathrm{Zn}_{x} \mathrm{MnO}_{2} \mathrm{ZHSCs}$ (Fig. 9a, b) clearly displayed XRD peaks of $\mathrm{Zn}_{4} \mathrm{SO}_{4}(\mathrm{OH})_{6} \cdot \mathrm{nH}_{2} \mathrm{O}$ and $\mathrm{ZnSO}_{3} \cdot 2.5\left(\mathrm{H}_{2} \mathrm{O}\right)$ at state $\mathrm{VI}$ and XIII, which correspond to the voltage of $2.0 \mathrm{~V}$. The by-products gradually precipitate during the charging process and dissolve during discharging period. Typically, this process was further demonstrated by the ex-situ SEM images shown in Fig. 9c. Also, the by-product of $\mathrm{Zn}_{4} \mathrm{SO}_{4}(\mathrm{OH})_{6} \cdot 0.5 \mathrm{H}_{2} \mathrm{O}$ is detected by Kang group on the surface of $\mathrm{ZBC}\left(\gamma-\mathrm{MnO}_{2}\right)$ [196]. On the contrary, the precipitation/dissolution processes are inverse since the Cap electrodes are applied as the cathode in the $\mathrm{Zn} / / \mathrm{Cap} \mathrm{ZHSCs}$, which was proved by the exsitu XPS spectra of carbon cathode depicted in Fig. 9d [77]. The $\mathrm{Zn}, \mathrm{O}, \mathrm{S}$ signals, which correspond to the formation of by-product $\mathrm{Zn}_{4} \mathrm{SO}_{4}(\mathrm{OH})_{6} \cdot \mathrm{nH}_{2} \mathrm{O}$, are enhanced by degrees during discharging process while the signals decreased during the charging process. Additionally, apart from the Cap electrodes, $\mathrm{Zn}_{4} \mathrm{SO}_{4}(\mathrm{OH})_{6} \cdot 5 \mathrm{H}_{2} \mathrm{O}$ can also be detected on the surface of $\mathrm{Zn}$ anodes [31, 82]. Typically, the reaction equation of by-products can be described as following Eqs. (3-3, 3-4) [34, 188]:

$4 \mathrm{Zn}^{2+}+6 \mathrm{OH}^{-}+\mathrm{SO}_{4}^{2-}+\mathrm{nH}_{2} \mathrm{O} \leftrightarrow \mathrm{Zn}_{4}(\mathrm{OH})_{6} \mathrm{SO}_{4} \cdot \mathrm{nH}_{2} \mathrm{O}$

$5 \mathrm{Zn}^{2+}+4 \mathrm{OH}^{-}+2 \mathrm{SO}_{4}^{2-}+4 \mathrm{H}_{2} \mathrm{O}+2 \mathrm{e}^{-} \leftrightarrow \mathrm{Zn}_{4}(\mathrm{OH})_{6} \mathrm{SO}_{4} \cdot 0.5 \mathrm{H}_{2} \mathrm{O}+\mathrm{ZnSO}_{3} \cdot 2.5\left(\mathrm{H}_{2} \mathrm{O}\right)$

Significantly, recently reported works ascribe this phenomenon to the change of $\mathrm{pH}$ value. As we know about the faintly acid property (initial $\mathrm{pH}$ of $\sim 4.5$ ) of $\mathrm{ZnSO}_{4}$ electrolyte, by-product formed when the $\mathrm{pH}$ value becomes higher than $~ 5.3$ and then dissolved when $\mathrm{pH}$ decreases [31]. Dong et al. supposed that the $\mathrm{pH}$ change results from the reversible hydrogen storage in nano-porous $\mathrm{AC}$ at a low potential, presented as Eqs. (3-5, 3-6) [35, 108]. Furthermore, Kang group particularly summarized two possible ways of causing the increase of $\mathrm{pH}$ in Cap//ZBC ZHSCs: (1) $\mathrm{H}^{+}$-decrease: the reaction between $\mathrm{H}^{+}$and $\mathrm{MnO}_{2}$ whereas the reaction is not dominant, (2) $\mathrm{OH}^{-}$-increase: water decomposition in nano-porous AC surface like Eq. (3-6) [188]. Typically, Zhang group found that the competitive hydrogen evolution process coexisted with $\mathrm{Zn}$ plating in $\mathrm{ZnSO}_{4}$ electrolyte below $0.2 \mathrm{~V}$ which can be the reason for $\mathrm{pH}$ increase [82]. Significantly, in our previous work of the ACC// $\mathrm{Zn}_{x} \mathrm{MnO}_{2} \mathrm{ZHSCs}$, ACC acts as anode and the deposition of $\mathrm{Zn}_{4} \mathrm{SO}_{4}(\mathrm{OH})_{6} \cdot \mathrm{nH}_{2} \mathrm{O}$ or $\mathrm{ZnSO}_{3} \cdot 2.5\left(\mathrm{H}_{2} \mathrm{O}\right)$ appeared only at the high voltage stage and then dissolved at low voltage (shown in Fig. 9c), it is believed that the high voltage can attract $\mathrm{Zn}^{2+}$ in $\mathrm{ZnSO}_{4}$ electrolyte and further form byproducts of $\mathrm{Zn}_{4} \mathrm{SO}_{4}(\mathrm{OH})_{6} \cdot \mathrm{nH}_{2} \mathrm{O}$ and $\mathrm{ZnSO}_{3} \cdot 2.5\left(\mathrm{H}_{2} \mathrm{O}\right)$ [34]. Briefly, the alteration of $\mathrm{pH}$ value is the outward manifestation and can be instructional for theoretical researches, more importantly, the hydroxyl/hydrogen ions and the adsorption of electrodes to the inverse cation/anion are the inner cause and mechanism of the precipitation/dissolution processes. Herein, we conclude this phenomenon as following results: (1) reactions in both cathodes and anodes which lead to the $\mathrm{pH}$ change, (2) the attraction to $\mathrm{Zn}^{2+} / \mathrm{SO}_{4}{ }^{2-}$ at different voltage stages in two configurations of ZHSCs. Typically, Eqs. (3-5, 3-6) are shown as follow [35, 108]:

$\mathrm{C}-\mathrm{OH} \leftrightarrow \mathrm{C}=\mathrm{O}+\mathrm{H}^{+}+\mathrm{e}^{-}$

$\mathrm{AC}+\mathrm{H}_{2} \mathrm{O}+\mathrm{e}^{-} \leftrightarrow \mathrm{AC} \cdot \mathrm{H}+\mathrm{OH}^{-}$

The influence of the by-products' formation can be divided into two steps. At first, the surface of electrodes is clean and easy to access electrolyte for energy storage processes. In this case, the $\mathrm{pH}$ value of the electrolyte stays low and the slow formation process of by-products can pro-

$$
5 \mathrm{Zn}^{2+}+4 \mathrm{OH}^{-}+2 \mathrm{SO}_{4}^{2-}+4 \mathrm{H}_{2} \mathrm{O}+2 \mathrm{e}^{-} \leftrightarrow \mathrm{Zn}_{4}(\mathrm{OH})_{6} \mathrm{SO}_{4} \cdot 0.5 \mathrm{H}_{2} \mathrm{O}+\mathrm{ZnSO}_{3} \cdot 2.5\left(\mathrm{H}_{2} \mathrm{O}\right)
$$

vide considerable capacity for the ZHSCs. However, after a short cycle, the reactions between the $\mathrm{H}^{+}$of electrolyte and electrodes increase the $\mathrm{pH}$ value of electrolyte, and then lead to the fast and uncontrollable formation process of byproducts. The uneven precipitation/dissolution process can 
greatly change the morphology of electrodes. Hence, due to the irreversible formation process of by-products, the ZHSCs can offer an excellent capacity at the first few cycles but suffer from the morphology modification for long cycles.

\subsection{Chemical Pseudocapacitive Reactions}

Only with the physical adsorption/desorption and precipitation/dissolution of by-products, there is no reason that the Cap electrodes are able to provide such high capacity which can even reach the capacity of whole redox-reaction-based batteries. When adopting the strategies of dopant, polymercarbon hybrid and other activated processes, the Cap electrodes are equipped with pseudocapacitive properties due to the functional group in the carbon skeleton and active structure/materials. The small redox peaks of Cap electrodes can be observed in the CV curves of many reported ZHSCs [77, 92, 108].

Significantly, Lu group captured more chemical transformation details of Cap electrodes which are depicted in Fig. 9e [85]. When the carbon electrodes were investigated by ex-situ XPS, the intensity of the $\mathrm{C}-\mathrm{OH}$ peak keeps decreasing from 1.8 to $0 \mathrm{~V}$ (A to $\mathrm{C}$, discharging process), and increasing from 0 to $1.8 \mathrm{~V}$ ( $\mathrm{C}$ to $\mathrm{E}$, charging process) whereas the $\mathrm{C}-\mathrm{O}-\mathrm{Zn}$ peak is reversed, indicating that there are reversible pseudocapacitive chemical reactions between the $\mathrm{C}-\mathrm{OH}$ and $\mathrm{C}-\mathrm{O}-\mathrm{Zn}$ bonds. Namely, the $\mathrm{C}-\mathrm{OH}$ bonds are chemically associated with $\mathrm{Zn}$-ion to form $\mathrm{C}-\mathrm{O}-\mathrm{Zn}$ bonds, which is the determining step in the entire chemical reactions. Typically, the ratio of the $\mathrm{C}-\mathrm{O}-\mathrm{Zn}$ to $\mathrm{C}-\mathrm{OH}$ at $0 \mathrm{~V}$ is generally related to the electrochemical performance, implying the extent of pseudocapacitive reactions between $\mathrm{C}-\mathrm{OH}$ and $\mathrm{Zn}$-ion. As shown in Fig. 9f, the ratio of HNPC electrode is $\sim 2.2$ times higher than the ratio of PC electrode which is close to the ratio of capacity ( 2.6 times), indicating the highly correlative relationship between the chemical pseudocapacitive reactions and ZHSCs capacity. Besides the $\mathrm{Zn}$-ions in the electrolyte, $\mathrm{H}^{+}$ions are also demonstrated to be a part of these reactions [77, 132]. Due to the faintly acid property of $\mathrm{ZnSO}_{4}$ electrolyte, $\mathrm{H}^{+}$ions generally exist in the electrolyte and take part in the chemical pseudocapacitive reactions of Cap electrodes and contribute considerable capacity to the ZHSCs. Meanwhile, it is also a crucial reason that the $\mathrm{OH}^{-}$concentration of electrolyte will increase, and further results in the precipitation/dissolution processes of by-products on the surface of various electrodes. The chemical pseudocapacitive reactions are presented as the following Eq. (3-7), and when N, B or other heteroatoms substitute part of $\mathrm{C}$ atoms in the carbon skeleton, the reactions proceed as Eq. (3-8), where the $\mathrm{X}$ represents element $\mathrm{N}$, B or other heteroatoms:

$$
\begin{aligned}
& \mathrm{C}-\mathrm{OH}+\mathrm{Zn}^{2+} / \mathrm{H}^{+}+\mathrm{e}^{-} \leftrightarrow \mathrm{C} \cdots \mathrm{O} \cdots \mathrm{Zn} / \mathrm{C} \cdots \mathrm{O} \cdots \mathrm{H} \\
& \mathrm{X}-\mathrm{OH}+\mathrm{Zn}^{2+} / \mathrm{H}^{+}+\mathrm{e}^{-} \leftrightarrow \mathrm{X} \cdots \mathrm{O} \cdots \mathrm{Zn} / \mathrm{X} \cdots \mathrm{O} \cdots \mathrm{H}
\end{aligned}
$$

In short, these reactions enable considerable capacity and abundant properties of Cap electrodes to realize better ZHSCs. Meanwhile, the combination of various energy storage mechanisms in Cap electrodes has been carefully analyzed. Even though, we deem it is necessary to further investigate and review the energy storage mechanisms of Cap electrodes. The kernel of the researches should be the balance between energy density and longevity, where the energy storage mechanisms can be the internal reasons for the external electrochemical performance.

\section{Electrolyte}

\subsection{Effects of Various Electrolyte Anions}

As the most frequently used anode in the $\mathrm{Zn} / / \mathrm{Cap}$ ZHSCs, $\mathrm{Zn}$ foils anodes possess similar size and thickness, but the corresponding $\mathrm{Zn}$-ion storage behaviors are diverse in different electrolytes [82]. The depositing/stripping behaviors of $\mathrm{Zn}$ anodes are highly related to the anions applied in the electrolytes. Generally, the cation is $\mathrm{Zn}^{2+}$ while the anions are diversiform, including $\mathrm{Cl}^{-}[34,153], \mathrm{SO}_{4}{ }^{2-}[31,53]$, $\mathrm{CF}_{3} \mathrm{SO}_{3}^{-}$[101], $\mathrm{ClO}_{4}^{-}[108], \mathrm{NO}_{3}{ }^{-}$and $\mathrm{CH}_{3} \mathrm{COO}^{-}$[140, 170], the interaction between the water molecules and electrolyte anions will affect the $\mathrm{Zn}$ depositing/stripping reactions and further determine the $\mathrm{Zn}$-ion storage efficiency as well as the electrochemical performance of ZHSCs. Here, we briefly review some reported researches about the effects of various electrolytes on ZHSCs [82].

As the exhibited SEM images and XRD spectra in Fig. 10a-f, Wu et al. investigated the plating morphology and phase composition of $\mathrm{Zn}-\mathrm{Ti}$ (Ti served as the substrate) anodes in five different electrolytes of $1.0 \mathrm{M} \mathrm{ZnSO}_{4}$, $\mathrm{Zn}\left(\mathrm{NO}_{3}\right)_{2}, \mathrm{Zn}\left(\mathrm{CH}_{3} \mathrm{COO}\right)_{2}, \mathrm{ZnCl}_{2}$ and $\mathrm{Zn}\left(\mathrm{CF}_{3} \mathrm{SO}_{3}\right)_{2}$ [82]. Surprisingly, the by-products of $\mathrm{Zn}_{4}\left(\mathrm{SO}_{4}\right)(\mathrm{OH})_{6} \cdot 5 \mathrm{H}_{2} \mathrm{O}$ and 

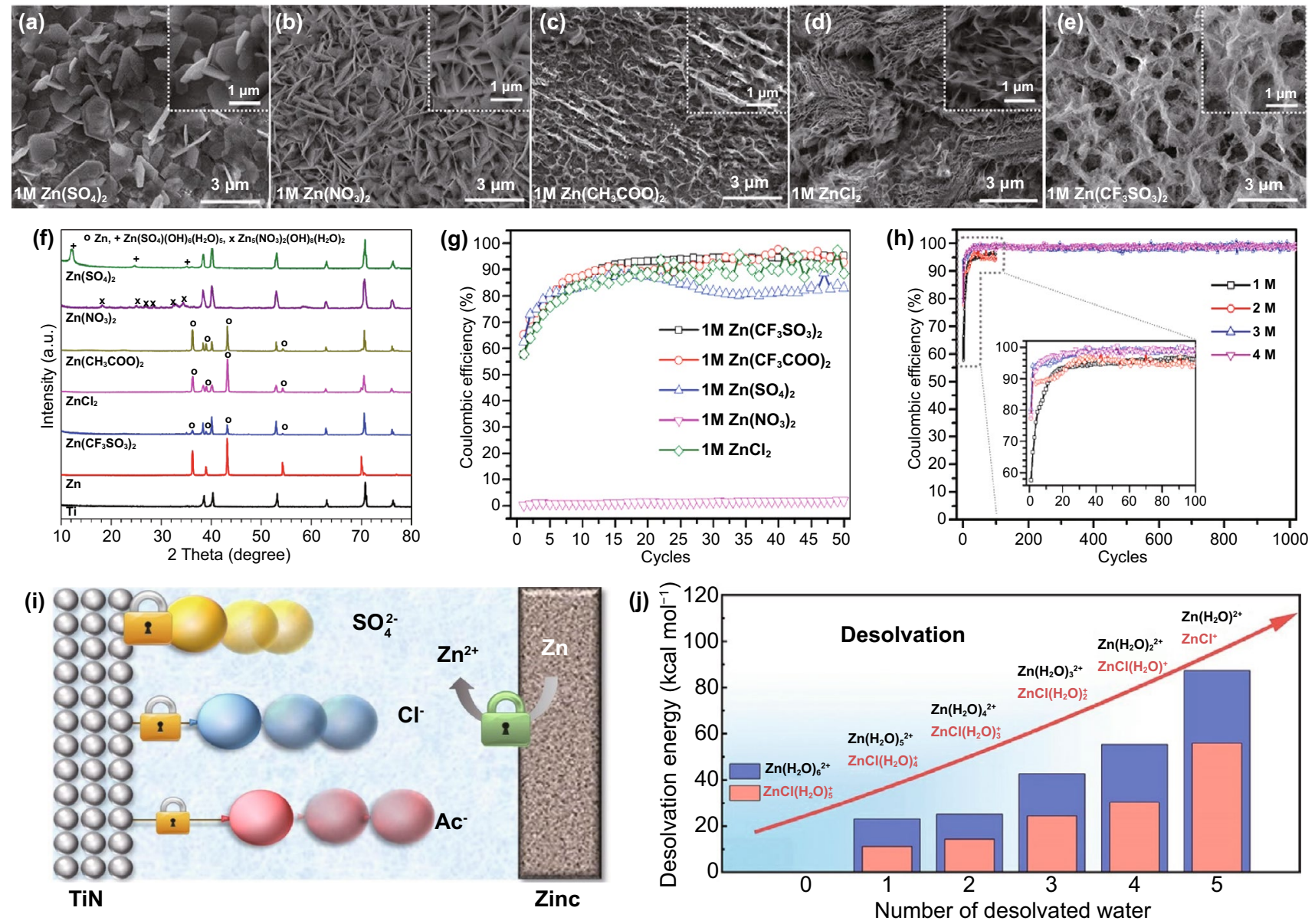

Fig. 10 a-e SEM images of pristine $\mathrm{Zn}$ and Ti foils with $\mathrm{Zn}$ layer plated in various electrolytes. $\mathbf{f}$ Corresponding XRD patterns and $\mathbf{g}$ coulombic efficiencies (CEs) of $\mathrm{Zn}$ depositing/stripping in various electrolytes. $\mathbf{h}$ CEs in $\mathrm{Zn}\left(\mathrm{CF}_{3} \mathrm{SO}_{3}\right)_{2}$ electrolytes with different concentrations. Reproduced with permission from Ref. [82]. Copyright 2019, Wiley-VCH. i Schematic illustration of self-discharge behaviors of Zn//TiN ZHSCs in three different electrolytes. Reproduced with permission from Ref. [169]. Copyright 2021, Wiley-VCH. j Desolvation energies of $\left[\mathrm{Zn}\left(\mathrm{H}_{2} \mathrm{O}\right)_{6}\right]^{2+}$ and $\left[\mathrm{ZnCl}\left(\mathrm{H}_{2} \mathrm{O}\right)_{5}\right]^{+}$. Reproduced with permission from Ref. [170]. Copyright 2021, Wiley-VCH

$\mathrm{Zn}_{5}\left(\mathrm{NO}_{3}\right)_{2}(\mathrm{OH})_{8} \cdot 2 \mathrm{H}_{2} \mathrm{O}$ were detected on the Ti substrates in $\mathrm{ZnSO}_{4}$ and $\mathrm{Zn}\left(\mathrm{NO}_{3}\right)_{2}$ electrolytes, respectively. No redundant peaks are observed in the XRD spectra of $\mathrm{Zn}$ anode when employed the $\mathrm{Zn}\left(\mathrm{CH}_{3} \mathrm{COO}\right)_{2}, \mathrm{ZnCl}_{2}$ and $\mathrm{Zn}\left(\mathrm{CF}_{3} \mathrm{SO}_{3}\right)_{2}$ as the electrolyte. Correspondingly, the SEM images of $\mathrm{Zn}$-Ti anodes in different electrolytes can be divided into two types. In the $\mathrm{ZnSO}_{4}$ and $\mathrm{Zn}\left(\mathrm{NO}_{3}\right)_{2}$ electrolytes (Fig. 10a, b), the deposited $\mathrm{Zn}-\mathrm{Ti}$ anodes presented morphology of nanosheets with random orientations while the porous and interconnected frame structure of deposited $\mathrm{Zn}-\mathrm{Ti}$ anodes are obtained in the $\mathrm{Zn}\left(\mathrm{CH}_{3} \mathrm{COO}\right)_{2}, \mathrm{ZnCl}_{2}$ and $\mathrm{Zn}\left(\mathrm{CF}_{3} \mathrm{SO}_{3}\right)_{2}$ electrolytes (Fig. 10c-e). Notably, the random stacked $\mathrm{Zn}$ nanosheets can be a possible reason for the formation of poor electrical conductivity by-products. Furthermore, the electrically isolated property of $\mathrm{Zn}$ nanosheets also leads to a high resistance of electrode, which can be verified by the CEs performance displayed in Fig. 10g. The Zn-Ti nanosheets presented almost zero $\mathrm{CE}$ in $\mathrm{Zn}\left(\mathrm{NO}_{3}\right)_{2}$ electrolyte, indicating an irreversible $\mathrm{Zn}$ depositing/stripping behavior. The $\mathrm{CE}$ of $\mathrm{Zn}$-Ti anode based on $\mathrm{ZnSO}_{4}$ electrolyte can maintain the same as the other three electrolytes at first 20 cycles, but then quickly declined to $\sim 80 \%$. Nevertheless, the CEs of $\mathrm{Zn}-\mathrm{Ti}$ anodes based on the other three electrolytes are relatively stable and gradually exceeded $90 \%$. Additionally, the impact of electrolyte concentration was investigated, for all these electrolytes, the CEs are promoted and stabilized with the increasing of electrolyte concentration. Among these electrolytes, the maximum CE of 
98.4\% is achieved by the $3.0 \mathrm{M} \mathrm{Zn}\left(\mathrm{CF}_{3} \mathrm{SO}_{3}\right)_{2}$ electrolyte, and the $\mathrm{CE}$ is saturated even further increase the concentration of $\mathrm{Zn}\left(\mathrm{CF}_{3} \mathrm{SO}_{3}\right)_{2}$ electrolyte. Moreover, as shown in Fig. 10h, $\mathrm{CE}$ of $3.0 \mathrm{M} \mathrm{Zn}\left(\mathrm{CF}_{3} \mathrm{SO}_{3}\right)_{2}$ electrolyte stably maintained for 1000 cycles, demonstrating the high reversibility of $\mathrm{Zn}$ depositing/stripping processes in $\mathrm{Zn}\left(\mathrm{CF}_{3} \mathrm{SO}_{3}\right)_{2}$ electrolyte. Comparably, saturated $\mathrm{Zn}\left(\mathrm{CH}_{3} \mathrm{COO}\right)_{2}$ electrolyte also reached a high $\mathrm{CE}$ of $\sim 95 \%$, which enable it a potential competitor to $\mathrm{Zn}\left(\mathrm{CF}_{3} \mathrm{SO}_{3}\right)_{2}$ electrolyte.

Generally, $\mathrm{ZnSO}_{4}$ and $\mathrm{Zn}\left(\mathrm{CF}_{3} \mathrm{SO}_{3}\right)_{2}$ are the most frequently used electrolytes in the reported ZHSCs researches, here we chiefly discuss these two electrolytes. On the one hand, by-products and nanosheets $\mathrm{Zn}$ layer may cause electrically isolated $\mathrm{Zn}$ and increase the resistance, and then lead to the inferior CEs in $\mathrm{ZnSO}_{4}$ electrolytes [82]. The by-products will generate on both cathode and anode, which makes an adverse impact on the cycling performance of ZHSCs. However, the by-product can provide considerable capacity for the ZHSCs at first few cycles and the capacity in $\mathrm{ZnSO}_{4}$ is relatively superior to the $\mathrm{ZHSCs}$ in $\mathrm{Zn}\left(\mathrm{CF}_{3} \mathrm{SO}_{3}\right)_{2}$ electrolyte [188]. On the other hand, as for the $\mathrm{Zn}\left(\mathrm{CF}_{3} \mathrm{SO}_{3}\right)_{2}$ electrolyte, evidences showed that the strong interaction between water molecules and $\mathrm{CF}_{3} \mathrm{SO}_{3}{ }^{-}$anions can recede the interaction between $\mathrm{Zn}$-ions and adjacent water molecules, thus suppressing the formation of by-products. The utilization of $\mathrm{Zn}\left(\mathrm{CF}_{3} \mathrm{SO}_{3}\right)_{2}$ electrolyte can improve the reversibility of the formation process and enable the ZHSCs with a high efficiency of $\mathrm{Zn}$ depositing/stripping processes as well as a long cycling lifetime. However, the formation of by-products cannot be entirely bypassed even in $\mathrm{Zn}\left(\mathrm{CF}_{3} \mathrm{SO}_{3}\right)_{2}$ electrolytes [197]. Summarily, the ZHSCs in $\mathrm{ZnSO}_{4}$ electrolyte can provide higher capacity but low efficiency as well as relatively inferior longevity. The $\mathrm{Zn}\left(\mathrm{CF}_{3} \mathrm{SO}_{3}\right)_{2}$ electrolyte shows faster kinetics and high-efficient $\mathrm{Zn}$ depositing/stripping processes while it can only provide a limited capacity and its price is too expensive (> 18 times than $\mathrm{ZnSO}_{4}$ electrolyte) [67].

Except for the effects on the $\mathrm{Zn}$ anode, the anion carriers also result in a significant difference in the Cap electrodes. To investigate the effects of anions carriers on the electrochemical energy storage behaviors of Cap electrodes, Huang et al. applied the $\mathrm{Zn} / / \mathrm{TiN}$ ZHSCs as a model in three different $\mathrm{Zn}$-ion salt electrolytes including $\mathrm{ZnSO}_{4}, \mathrm{ZnAc}_{2}$ and $\mathrm{ZnCl}_{2}$ [169]. After 900 cycles tests, the $\mathrm{Zn} / / \mathrm{TiN}$ ZHSCs in $\mathrm{ZnSO}_{4}$ presented the highest capacity of $405.1 \mathrm{~F} \mathrm{~g}^{-1}$ at a current density of $0.5 \mathrm{~A} \mathrm{~g} \mathrm{~g}^{-1}$ among three $\mathrm{Zn}$-ion salt electrolytes while the other two with $\mathrm{ZnAc}_{2}$ and $\mathrm{ZnCl}_{2}$ only show
162.3 and $245.9 \mathrm{~F} \mathrm{~g}^{-1}$, respectively. The capacity difference is ascribed to the adsorption energy between anions and TiN cathodes, where the $\mathrm{SO}_{4}{ }^{2-}$ showed a more negative adsorption energy of $-6.3942 \mathrm{~V}$, which is almost two times larger than $\mathrm{Cl}^{-}$and $\mathrm{Ac}^{-}$. Moreover, the negative adsorption energy of $\mathrm{SO}_{4}{ }^{2-}$ can lead to a more stable structure of $\mathrm{TiN}-\mathrm{SO}_{4}$ after adsorption process. As illustrated in Fig. 10i, the stable structure of $\mathrm{TiN}^{-\mathrm{SO}_{4}}$ also make a significant contribution to the anti-self-discharge ability of Zn//TiN ZHSCs. Since the $\mathrm{Zn}$ anode would not spontaneously convert to $\mathrm{Zn}$-ions, the adsorption interaction between the anions and TiN cathode is the hinge to inhibit the self-discharge behaviors. With the stable lock of TiN-SO $\mathrm{S}_{4}$ structure, the $\mathrm{Zn} / / \mathrm{TiN} \mathrm{ZHSCs}$ offered an excellent self-discharge performance of $83.92 \%$ capacity retention after $500 \mathrm{~h}$ resting time.

In another work, Wang et al. demonstrated the great influence of water molecules on the $\mathrm{Zn}$-ion electrolytes, where the diameter of $\mathrm{Zn}$-ion can vastly increase from $1.48 \AA$ (bare $\left.\mathrm{Zn}^{2+}\right)$ to $8.60 \AA\left(\left[\mathrm{Zn}\left(\mathrm{H}_{2} \mathrm{O}\right)_{6}\right]^{2+}\right)$ [170]. The large diameter of hydrated $\mathrm{Zn}$-ions may lead to a significant loss of capacity due to ion-sieving effects of porous carbon electrodes. Since the cation is $\mathrm{Zn}$-ion, the anions in different $\mathrm{Zn}$-ion electrolytes are crucial parts to promote the desolvation of $\mathrm{Zn}$-ions and enhance the capacity of ZHSCs. Hence, Wang et al. investigated various $\mathrm{Zn}$ salt electrolytes and demonstrated that the $\mathrm{ZnCl}_{2}$ salt electrolyte is an optimal choice for desolvation of $\mathrm{Zn}$-ions. As shown in Fig. 10j, the density functional theory (DFT) calculation results presented that the desolvation energy required by $\left[\mathrm{ZnCl}\left(\mathrm{H}_{2} \mathrm{O}\right)_{5}\right]^{+}$is only half of that required by $\left[\mathrm{Zn}\left(\mathrm{H}_{2} \mathrm{O}\right)_{6}\right]^{2+}$, thus, the $\left[\mathrm{ZnCl}\left(\mathrm{H}_{2} \mathrm{O}\right)_{5}\right]^{+}$can easily lose water molecule and get access to the micropores of carbon electrodes. Among the applied $\mathrm{Zn}$ salt electrolytes, the $\mathrm{ZnCl}_{2}$-based ZHSCs reached the highest capacity of $229.4 \mathrm{~F} \mathrm{~g} \mathrm{~g}^{-1}$ at $1 \mathrm{~A} \mathrm{~g}^{-1}$. Furthermore, $\mathrm{ZnCl}_{2}$ electrolytes enable the smallest $R_{\mathrm{ct}}$ and the fastest ion exchange rates among various electrolytes. Notably, a remarkable cycling performance of $95.1 \%$ capacity retention was realized based on the $\mathrm{ZnCl}_{2} / \mathrm{PAM}$ hydrogel electrolyte after incredible 100,000 cycles, together with good low-temperature adaptability (only $7.1 \%$ capacity decay after 40,000 cycles at $-20^{\circ} \mathrm{C}$ ) and excellent mechanical flexibility.

By far, the most frequently used electrolytes in reported ZHSCs are $\mathrm{ZnSO}_{4}$ and $\mathrm{Zn}\left(\mathrm{CF}_{3} \mathrm{SO}_{3}\right)_{2}$, however, the dilemma of $\mathrm{ZnSO}_{4}$ and $\mathrm{Zn}\left(\mathrm{CF}_{3} \mathrm{SO}_{3}\right)_{2}$ may limit the development of high-performance ZHSCs. Here, we strongly believe that some novel electrolytes like $\mathrm{Zn}\left(\mathrm{CH}_{3} \mathrm{COO}\right)_{2}$, 
$\mathrm{Zn}\left(\mathrm{ClO}_{4}\right)_{2}$ and $\mathrm{ZnCl}_{2}$ can be the choices to promote the combination of high-performance, long cycling lifetime and by-product-free ZHSCs. For example, Xu et al. reported $\mathrm{Zn} / / 1.0 \mathrm{M} \mathrm{Zn}\left(\mathrm{CH}_{3} \mathrm{COO}\right)_{2} / / \mathrm{rGO} / \mathrm{PPD} \mathrm{ZHSCs}$ with an ultra-high areal capacity of $\sim 3 \mathrm{~F} \mathrm{~cm}^{-2}$ at a current density of $1 \mathrm{~mA} \mathrm{~cm}^{-2}$, and outstanding cycling stability which maintains $100 \%$ of initial capacity after 4000 cycles [140]. Additionally, Yin et al. assembled the Zn//PC800 ZHSCs in the $3.0 \mathrm{M} \mathrm{Zn}\left(\mathrm{ClO}_{4}\right)_{2}$ electrolyte, presenting a wide operating voltage window $(0-1.9 \mathrm{~V})$ and prominent capacity of $179.8 \mathrm{mAh} \mathrm{g}^{-1}$ as well as superb capacity retention of $99.2 \%$ after 30,000 cycles [108]. Moreover, $\mathrm{Li}$ et al. prepared the $\mathrm{Zn}-\mathrm{Ti}_{3} \mathrm{C}_{2} \mathrm{~T}_{x} / / \mathrm{Ti}_{3} \mathrm{C}_{2} \mathrm{~T}_{x} \mathrm{ZmSCs}$ in $6.0 \mathrm{M}$ $\mathrm{ZnCl}_{2}$ gel electrolyte, and delivered a remarkable cycling performance of $79.6 \%$ capacity retention after 50,000 cycles [153]. Therefore, more attentions should be focused on these novel electrolytes and the interactions between the electrolyte anions and both electrodes, which may promote the realization of the balanced, high-performance and high-efficient ZHSCs.
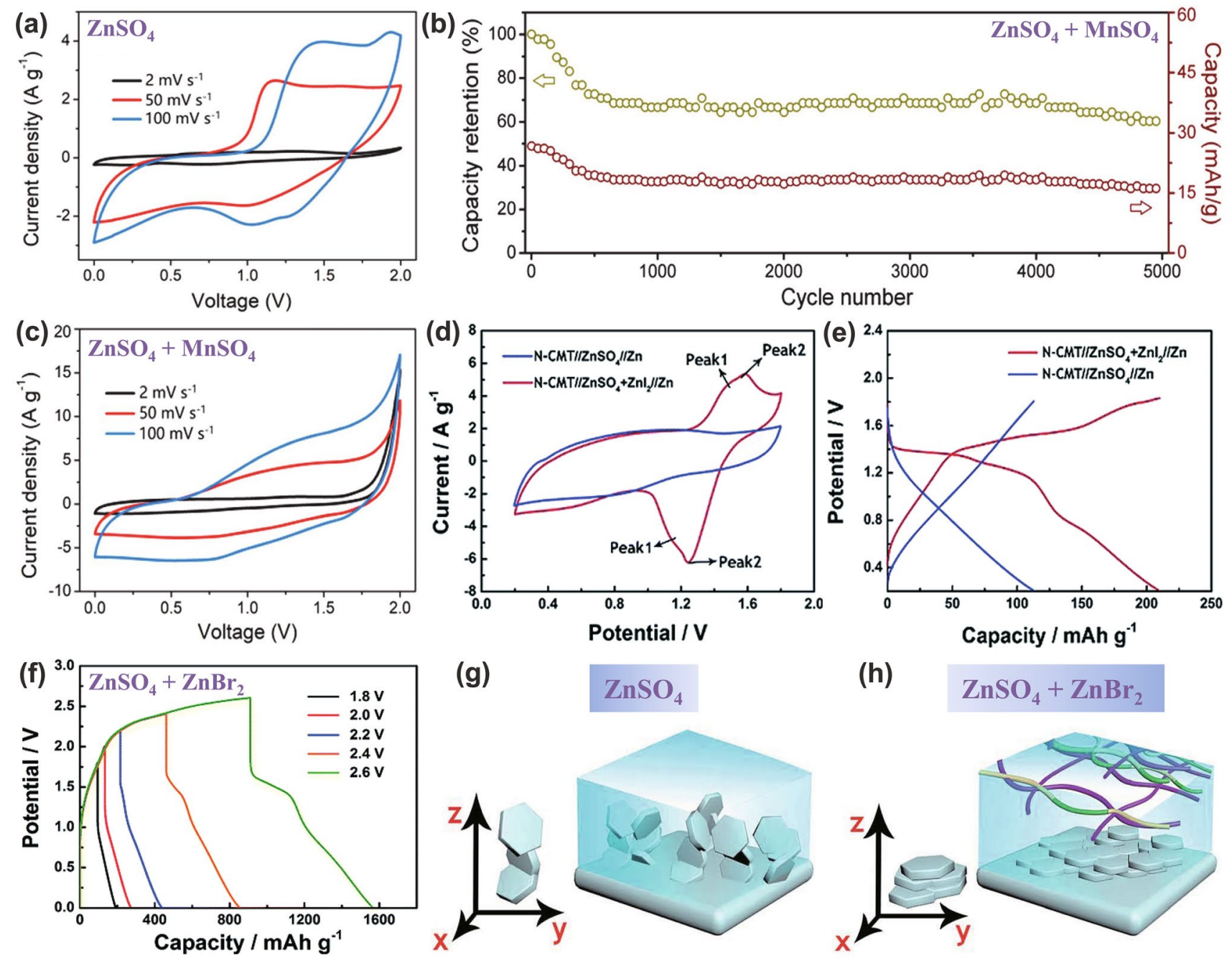

Fig. 11 a $\mathrm{CV}$ curves of $\mathrm{AC} / / 2.0 \mathrm{M} \mathrm{ZnSO}_{4} / / \gamma-\mathrm{MnO}_{2}$ ZHSCs. b Cycling performance and c CV curves of $\mathrm{AC} / / 2.0 \mathrm{M} \mathrm{ZnSO} 4+0.5 \mathrm{M}$ $\mathrm{MnSO}_{4} / / \gamma-\mathrm{MnO}_{2} \mathrm{ZHSCs}$. Reproduced with permission from Ref. [188]. Copyright 2019, Elsevier. d CV curves and e GCD profiles of Zn//NCMT ZHSCs in different electrolytes. Reproduced with permission from Ref. [110]. Copyright 2019, Royal Society of Chemistry. f GCD curves with diverse voltage windows. $\mathbf{g}$, $\mathbf{h}$ Schematic illustration of $\mathrm{Zn}$ deposition in $\mathbf{g} \mathrm{ZnSO}_{4}$ and $\mathbf{h} \mathrm{SA}-\mathrm{Zn}-\mathrm{Br}$ hydrogel electrolytes. Reproduced with permission from Ref. [198]. Copyright 2020, Royal Society of Chemistry 


\subsection{Quasi-Solid-state Electrolyte}

With the rapid development of wearable electronic devices, flexible ESSs are in great demands. As the most important part of flexible ESSs, the electrolyte featured with flexibility, high mechanical strength, excellent wettability and high safety can enable flexible ESSs with the same properties as the aqueous systems. However, the relatively low electrical conductivity is still an obstacle for high-performance flexible devices. Due to the fast kinetic of ZHSCs, the solid-state electrolyte with insufficient conductivity is inappropriate for flexible ZHSCs devices. Instead, the quasi-solid-state or gel electrolytes are suitable candidates for flexible ZHSCs devices. Typically, the gel electrolytes of reported flexible ZHSCs include the zinc salts $\left(\mathrm{ZnCl}_{2}\right.$ [34, 153], $\mathrm{ZnSO}_{4}$ [77], etc.), additive ( $\mathrm{MnSO}_{4}$ [34], $\mathrm{ZnBr}_{2}$ [198], etc.), polymeric frameworks (PVA [85], PAA [120], PAM [99], gelatin [83, 96], etc.) and a small amount of water. To some extent, the gel electrolytes can suppress some side effects, such as the dissolution of electrodes, $\mathrm{Zn}$ dendrite and water decomposition [67]. Notably, ethylene glycol (EG), as a common used antifreeze, was applied as an additive in the quasi-solidstate electrolyte for anti-freezing and low-temperature-tolerant flexible ZHSCs, owing to the high boiling point, high dielectric constant and splendid solubility in water [197]. The reported durable, flexible ZHSCs devices can incredibly work at ultra-low temperatures from -15 to $-40{ }^{\circ} \mathrm{C}$ $[199,200]$.

\subsection{Additive Electrolyte}

Additive electrolytes are the common strategies to further stabilize or promote the electrochemical performance of many ESSs [201, 202]. With the synergy effects of various cations or anions, the energy storage behaviors are modified, and then excellent electrochemical performances can be obtained. Among the numerous additive electrolytes, $\mathrm{MnSO}_{4}$ is a superb choice for the stabilization of $\mathrm{MnO}_{2}$ in ZIBs [67]. For instance, Kang group investigated the effect of $\mathrm{MnSO}_{4}$ additive electrolyte in the $\mathrm{AC} / / \gamma-\mathrm{MnO}_{2} \mathrm{ZHSCs}$ system [188]. Figure 11a exhibits the asymmetric CV curves of $\mathrm{AC} / / \gamma-\mathrm{MnO}_{2} \mathrm{ZHSCs}$ in $2.0 \mathrm{M} \mathrm{ZnSO}_{4}$ electrolyte, indicating the typical $\mathrm{Zn}$-ion insertion/desertion redox reactions of $\gamma-\mathrm{MnO}_{2}$ cathode. Due to the high dissolvability and poor electrical conductivity of manganese-based materials, the $\mathrm{AC} / / \gamma-\mathrm{MnO}_{2} \mathrm{ZHSCs}$ can merely keep $65.3 \%$ of initial capacity after short 3000 cycles without the $\mathrm{MnSO}_{4}$ additive electrolyte. However, when applied the $0.5 \mathrm{M} \mathrm{MnSO}_{4}$ as the additive electrolyte, the addition of $\mathrm{Mn}^{2+}$ provided a positive effect on the cycling performance, the ZHSCs presented a retention of $61.8 \%$ after prolonged 5000 cycles where the capacity decay quite slow after the first 500 cycles (Fig. 11b). After first 500 cycles, the capacity retention was measured to be $\sim 88 \%$ for 4500 cycles. In addition, the ACC// $\mathrm{Zn}_{x} \mathrm{MnO}_{2} \mathrm{ZHSCs}$ assembled by Mai group can even achieve $83.1 \%$ capacity retention after 5000 cycles based on $0.4 \mathrm{M}$ $\mathrm{MnSO}_{4}$ additive electrolyte [34].

Hence, it is believed that the utilization of $\mathrm{MnSO}_{4}$ additive electrolyte can effectively restrain the dissolution process of $\mathrm{MnO}_{2}$ by equilibrating the $\mathrm{Mn}$ element between $\mathrm{MnO}_{2}$ electrodes and electrolytes [203]. Additionally, as exhibited in Fig. 11c, the $\mathrm{Zn}$-ion energy storage behavior of $\gamma-\mathrm{MnO}_{2}$ electrodes is changed due to the addition of $\mathrm{Mn}^{2+}$, the $\mathrm{CV}$ curves become more symmetrical and distinct polarization peaks are observed near to $2.0 \mathrm{~V}$. Compared with the non$\mathrm{Mn}^{2+} \mathrm{ZHSC}$ system, the capacity of $\mathrm{AC} / / \gamma-\mathrm{MnO}_{2} \mathrm{ZHSCs}$ in the mixed electrolyte of $2.0 \mathrm{M} \mathrm{ZnSO}_{4}$ and $0.5 \mathrm{M} \mathrm{MnSO}_{4}$ was improved from 54.1 to $83.8 \mathrm{mAh} \mathrm{g}^{-1}$. Many reported studies demonstrated that the $\mathrm{Mn}^{2+}$ of the $\mathrm{MnSO}_{4}$ additive electrolyte also take part in the energy storage reactions of $\mathrm{MnO}_{2}$ cathode. By oxidizing to $\mathrm{MnO}_{2}$ and further depositing on the surface of $\mathrm{MnO}_{2}$ cathode, the $\mathrm{Mn}^{2+}$ gradually become the additional host electrode for $\mathrm{Zn}$-ion storage [203, 204].

As we reviewed in Sect. 4.1, the significant difference of electrolyte anions may cause distinct energy storage behaviors of ZHSCs. Therefore, the anion additive electrolyte can be a big variate for enhancing the electrochemical performance of ZHSCs. Han et al. applied 0.01 M zinc iodide $\left(\mathrm{ZnI}_{2}\right)$ as the additive electrolyte, then the as-prepared Zn//N-CMT ZHSCs delivered a remarkable capacity of $416.6 \mathrm{mAh} \mathrm{g}^{-1}$ at $1 \mathrm{~A} \mathrm{~g}^{-1}$, which is almost two times higher than the capacity of $\mathrm{I}^{-}$-free ZHSCs [110]. As shown in Fig. 11d, the CV curves present diverse shapes of $\mathrm{Zn} / / \mathrm{N}-\mathrm{CMT} \mathrm{ZHSCs}$ in the $\mathrm{ZnSO}_{4}$ and $\mathrm{ZnSO}_{4}+\mathrm{ZnI}_{2}$ electrolytes. Without the $\mathrm{ZnI}_{2}$ additive electrolyte, the $\mathrm{Zn} / \mathrm{N}$ CMT ZHSCs showed distorted rectangular CV curve and no redox peak, indicating the leading role of EDLC. When employed the $\mathrm{ZnI}_{2}$ additive electrolyte, the evident redox peaks can be detected at approximately 1.2 and $1.5 \mathrm{~V}$. Typically, each peak contains two tiny peaks where the peak 1 refers to the reaction of $2 \mathrm{I}^{-} / \mathrm{I}_{2}$ and the peak 2 correspond to 
the reaction of $3 \mathrm{I}^{-} / \mathrm{I}_{3}{ }^{-}[205,206]$. The energy storage reactions are enriched due to the addition of $\mathrm{I}^{-}$and the redox reactions of $\mathrm{I}^{-}$generally occur on the surface of N-CMT. In addition, $\mathrm{I}^{-}$added ZHSCs exhibited obvious charging/discharging platforms at $\sim 1.4 \mathrm{~V}$ (Fig. 11e). A small amount of $\mathrm{I}^{-}$additive can tremendously enhance the capacity of ZHSCs but not sacrifice the power performance, the $\mathrm{Zn} / / \mathrm{BN}-\mathrm{CMTs}$ ZHSCs with $\mathrm{I}^{-}$additive can offer the second-highest energy density of $472.6 \mathrm{Wh} \mathrm{kg}^{-1}$ at an incredible power density of $1600 \mathrm{~W} \mathrm{~kg}^{-1}$. Also, providing a peak power density of $16,000 \mathrm{~W} \mathrm{~kg}^{-1}$ at high current density. The redox reaction equations of iodide are presented as following Eqs. (4-1, 4-2) [198]:

$\mathrm{I}_{2}+2 \mathrm{e}^{-} \leftrightarrow 2 \mathrm{I}^{-}$

$\mathrm{I}_{3}^{-}+2 \mathrm{e}^{-} \leftrightarrow 3 \mathrm{I}^{-}$

Subsequently, by further tuning of halogen elements, Han et al. replaced the $\mathrm{ZnI}_{2}$ additive electrolyte with zinc bromide $\left(\mathrm{ZnBr}_{2}\right)$, then the highest energy density and ultrahigh voltage window of $2.6 \mathrm{~V}$ as well as controllable $\mathrm{Zn}$ deposition of $\mathrm{Zn} / / \mathrm{AC} \mathrm{ZHSCs}$ were achieved [198]. Sodium alginate (SA), $\mathrm{ZnSO}_{4}$ and $\mathrm{ZnBr}_{2}$ were employed to form the $\mathrm{SA}-\mathrm{Zn}$-Br hydrogel electrolyte. Unlike the symmetrical CV curve of $\mathrm{I}^{-}$added $\mathrm{ZHSCs}$, the $\mathrm{Br}^{-}$added ZHSCs presented evident polarizations. The asymmetric $\mathrm{CV}$ indicated the irreversible redox reactions of $\mathrm{Br}^{-}$added ZHSCs. Moreover, the $\mathrm{Br}^{-}$added flexible ZHSCs devices kept a capacity retention of $87.7 \%$ after 5000 cycles, which is inferior to the $99.3 \%$ capacity retention of $\mathrm{I}^{-}$added ZHSCs after 10,000 cycles. Even though, the $\mathrm{Br}^{-}$additive electrolyte provided ZHSCs with the ultra-high voltage windows above normal $1.8 \mathrm{~V}$, as depicted in the GCD plots of Fig. 11f. Although the CE of ZHSCs signally decreased due to the over-high voltage of $2.6 \mathrm{~V}$, the charging/discharging behavior can be balanced at $2.4 \mathrm{~V}$, which is still much higher than reported ZHSC systems. On account of the ultra-high voltage window, the assembled Br-added ZHSCs offered a maximum energy density of $605 \mathrm{Wh} \mathrm{kg}^{-1}$ at the power density of $1848 \mathrm{~W} \mathrm{~kg}^{-1}$. The energy density will decrease to $390.9 \mathrm{Wh} \mathrm{kg}^{-1}$ when lower the voltage window to $2.4 \mathrm{~V}$. More importantly, the application of SA-Zn-Br electrolyte enabled the $\mathrm{Zn}$ deposition more controllable. As reviewed in Sect. 4.1, $\mathrm{Zn}$ is deposited as the nanosheets with random orientations in $\mathrm{ZnSO}_{4}$ electrolytes, where the schematic is illustrated in Fig. 11g. However, due to the interactions between charged groups and $\mathrm{Zn}$-ions, $\mathrm{Br}^{-}$can efficiently harmonize the migration of $\mathrm{Zn}$-ion with uniform nucleation on $\mathrm{Zn}$ foil anodes [207, 208], and serve as an inhibitor of water/oxygen to avoid the corrosion of $\mathrm{Zn}$ foils. Therefore, as depicted in Fig. 11h, layered $\mathrm{Zn}$ depositions are controlled by the $\mathrm{Br}^{-}$additive electrolyte. Similarly, the $\mathrm{Br}^{-}$redox reaction equations are presented as following Eqs. (4-3, 4-4) [198]:

$$
\begin{aligned}
& \mathrm{Br}_{2}+2 \mathrm{e}^{-} \leftrightarrow 2 \mathrm{Br}^{-} \\
& 2 \mathrm{Br}_{2}+2 \mathrm{e}^{-} \leftrightarrow \mathrm{Br}_{3}^{-}+\mathrm{Br}^{-}
\end{aligned}
$$

\section{Zn-Ion Hybrid Micro-Supercapacitors}

Apart from aqueous ZHSCs system, many high-performance ZmSCs have been realized based on the in-plane electrochemical energy storage [32, 97, 153, 209, 210]. By far, only the electrode configuration of $\mathrm{Zn} / / \mathrm{Cap} \mathrm{ZmSCs}$ are reported. Notably, the reported ZmSCs can achieve higher capacities than most traditional micro-supercapacitors (mSCs), including symmetric/asymmetric micro-supercapacitors and novel Li-ion micro-supercapacitors (SmSCs, AmSCs and LmSCs) [211-219]. Table 3 summarized the electrochemical performance of various reported micro-supercapacitors. Besides the capacity, the overall performances of $\mathrm{ZmSCs}$ is superior to other mSCs devices.

For one, Feng group fabricated $\mathrm{ZmSCs}$ where the electrodeposited $\mathrm{Zn}$ nanosheets act as the anodes and the $\mathrm{AC}$ on the opposite electrode act as cathodes in the $\mathrm{ZnSO}_{4}$ aqueous electrolyte [32]. Figure 12a exhibits the standard lithography processes involved in the $\mathrm{Zn} / / \mathrm{AC} \mathrm{ZmSCs}$, and the optical images of as-assembled $\mathrm{ZmSCs}$ before and after packaging are shown in Fig. 12b. Remarkably, the Zn//AC ZmSCs provided an ultra-high areal capacity of $1297 \mathrm{mF} \mathrm{cm}^{-2}$ at $0.16 \mathrm{~mA} \mathrm{~cm}^{-2}$, which is almost 5-500 times higher than SmSCs and AmSC. Meanwhile, the energy density of the ZmSCs reached $115.4 \mu \mathrm{Wh} \mathrm{cm}{ }^{-2}$ at a power density of 0.16 $\mathrm{mW} \mathrm{cm}{ }^{-2}$. In addition, it presented an ultra-stable cycling performance with $100 \%$ capacity retention after 10,000 cycles at $1.56 \mathrm{~mA} \mathrm{~cm}^{-2}$, which can be further confirmed by the symmetric rectangular shape of CV curves (Fig. 12c) at various scan rates of $0.2-5 \mathrm{mV} \mathrm{s}^{-1}$. For another, Sun et al. assembled $\mathrm{Zn} / / \mathrm{CNT} \mathrm{ZmSCs}$ with recoverable $\mathrm{Zn}$ anodes and the fabrication processes are shown in Fig. 12d [97]. The CNT paper was firstly pasted onto the polyimide tape 


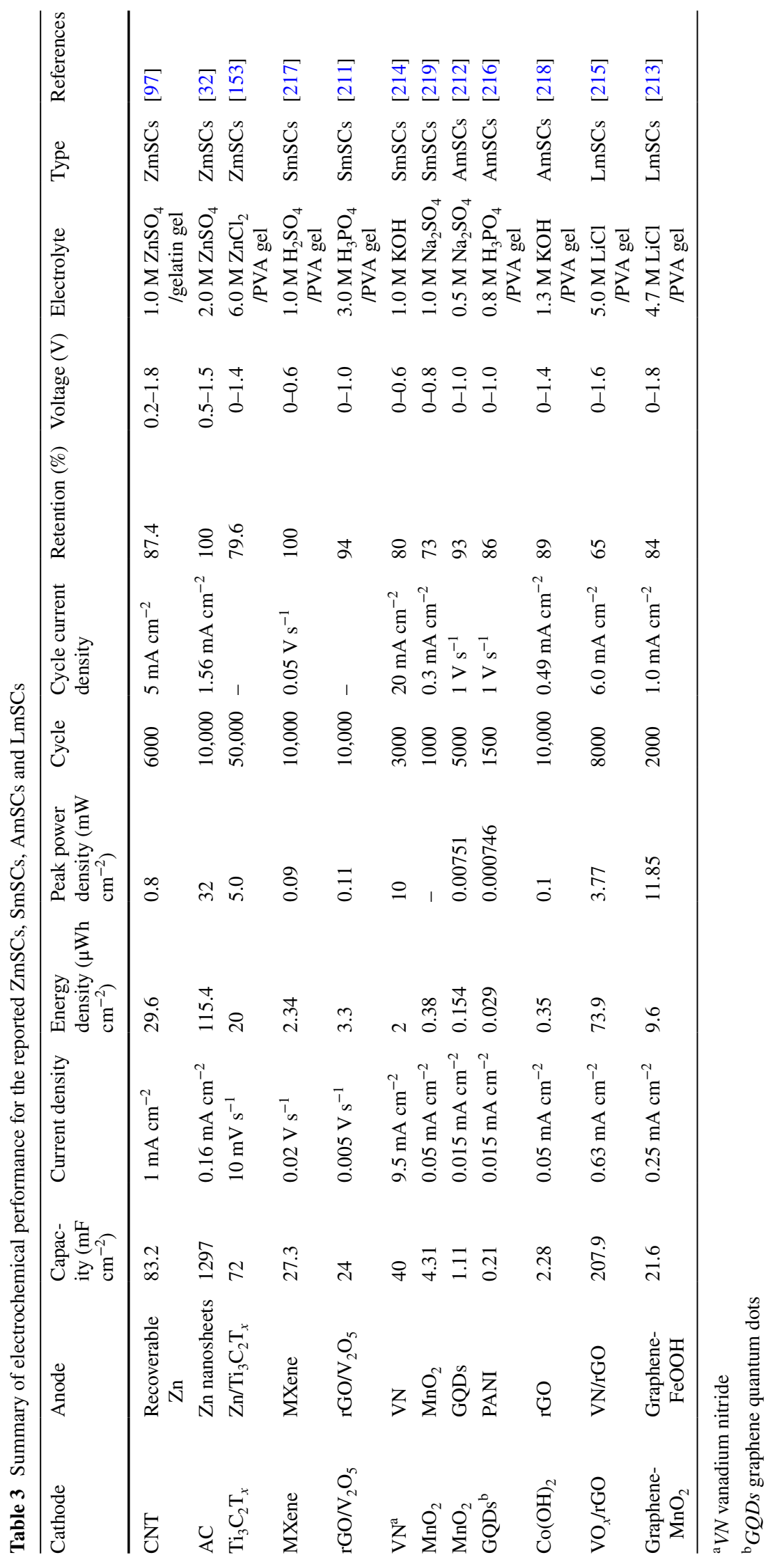


(a)

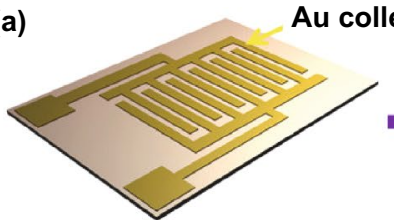

(i) Ti/Au patterning

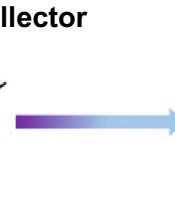

(ii)

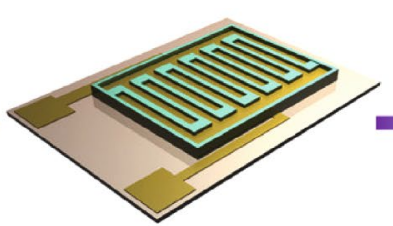

ii) Photoresist patterning
Zn nanosheet

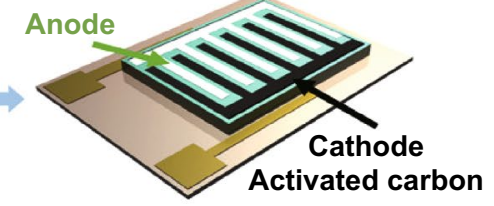

(iii) Active materials

electrodeposition and injection
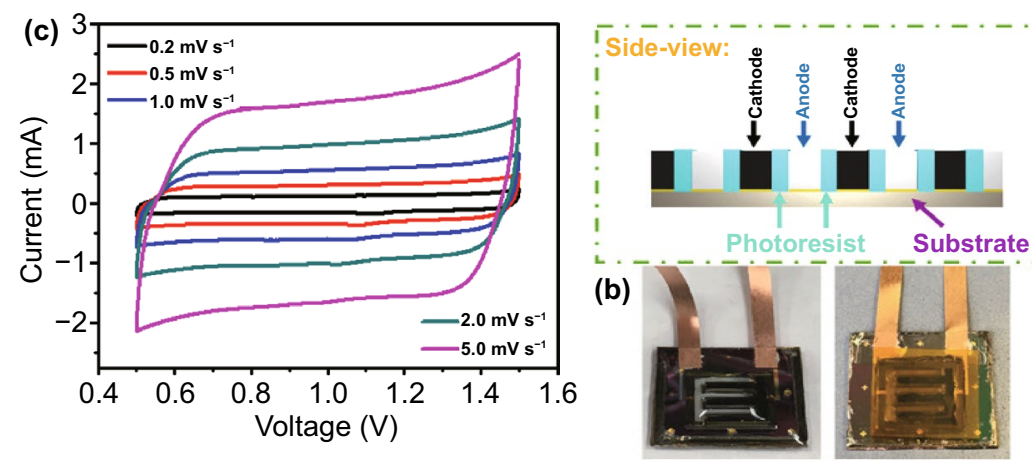

(d)
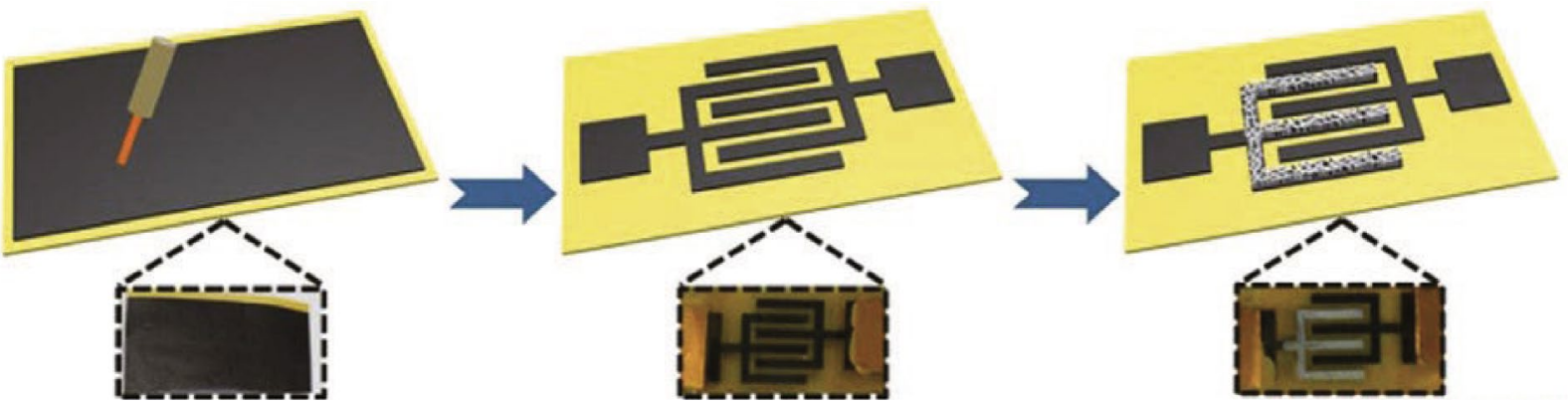

(e)

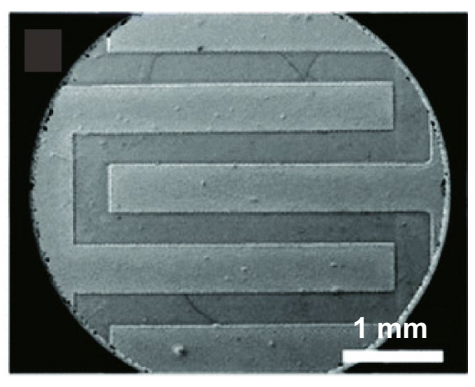

(f)

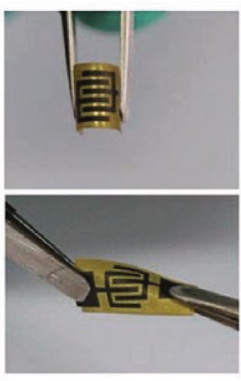

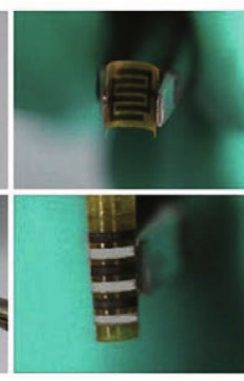

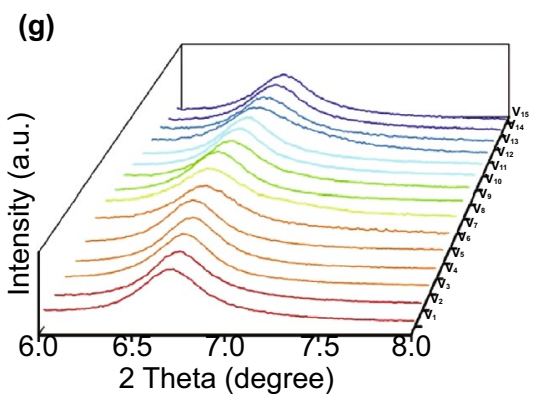

Fig. 12 a Fabrication process, b optical images before and after packaging and $\mathbf{c}$ CV curves of the Zn//AC ZmSCs. Reproduced with permission from Ref. [32]. Copyright 2019, Wiley-VCH. d Preparation processes of the Zn//CNT ZmSCs. e SEM image of microelectrode. f Photographs of the Zn//CNT ZmSCs at the bent and twisted states. Reproduced with permission from Ref. [97]. Copyright 2018, Royal Society of Chemistry. $g$ Ex-situ XRD spectra of $\mathrm{Ti}_{3} \mathrm{C}_{2} \mathrm{~T}_{x}$ cathode in ZmSCs. Reproduced with permission from Ref. [153]. Copyright 2021, Springer Nature

and then patterned interdigital microelectrodes employing a computer-controlled laser cutting system. The SEM image of microelectrode is displayed in Fig. 12e. Typically, the measured areal energy density of the $\mathrm{Zn} / / \mathrm{CNT} \mathrm{ZmSCs}$ is 29.6 $\mu \mathrm{Wh} \mathrm{cm}^{-2}$ and the peak power density is $8 \mathrm{~mW} \mathrm{~cm}^{-2}$. Furthermore, the flexible $\mathrm{ZmSCs}$ devices are also investigated by introducing the $\mathrm{ZnSO}_{4}$ gel electrolyte, the digital photos of $\mathrm{Zn} / / \mathrm{CNT} \mathrm{ZmSCs}$ devices at the bent and twisted states are depicted in Fig. 12f, indicating the splendid mechanical flexibility of flexible ZmSCs devices. Remarkably, the ZmSCs can offer stable GCD curves at different degrees of inflection and maintained excellent capacity retention of $89.5 \%$ after 
1000 mild bending cycles. Lately, Shen group applied 2D layered $\mathrm{Ti}_{3} \mathrm{C}_{2} \mathrm{~T}_{x}$ as the cathode of $\mathrm{ZmSCs}$ and realized great longevity of 50,000 cycles, keeping a capacity retention of $79.6 \%$ [153]. Significantly, the energy storage mechanisms of as-prepared ZmSCs were investigated by ex-situ XRD spectra shown in Fig. 12g, the characteristic peak (002) of $\mathrm{Ti}_{3} \mathrm{C}_{2} \mathrm{~T}_{x}$ shifted to the left by degree during discharging processes. According to Bragg's law $(2 d \sin \theta=n \lambda)$, the decrease of the peak (002) degree refers to the increase of interlayer spacing due to $\mathrm{Zn}$-ion insertion. On the contrary, the peak gradually moved to the initial degree during the charging process. Therefore, the $\mathrm{Zn}$-ions insertion/desertion processes display similar energy storage behaviors as aqueous ZHSCs.

With the miniaturization of electronic devices, the demands for micro electronic devices are growing, like wireless headsets and micro-ESSs. Therefore, it is believed that the study of ZmSCs will become a hot spot and bring a thorough revolution to mSCs research area. However, up to now, the studies of $\mathrm{ZmSCs}$ are lacking and short of an in-depth understanding of the unique in-plane energy storage behaviors. From our point of view, we deem that more suitable electrode materials should be attempted for $\mathrm{ZmSCs}$ (a)

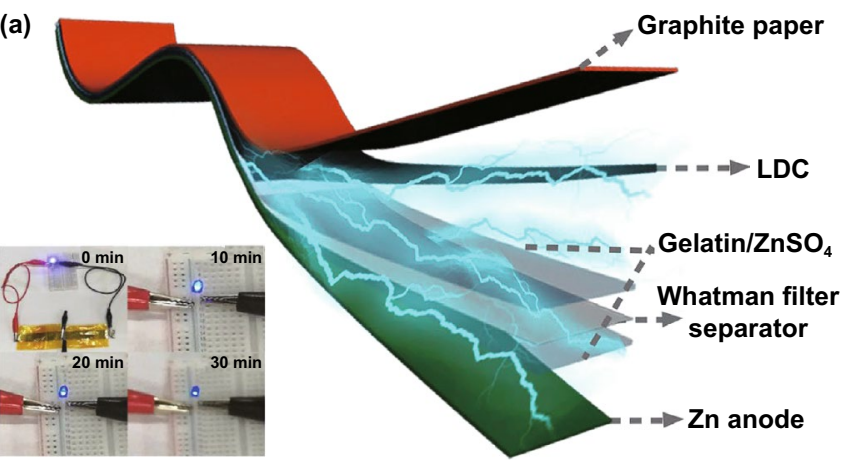

(c) Anode electrode (2D-Zn@CF)

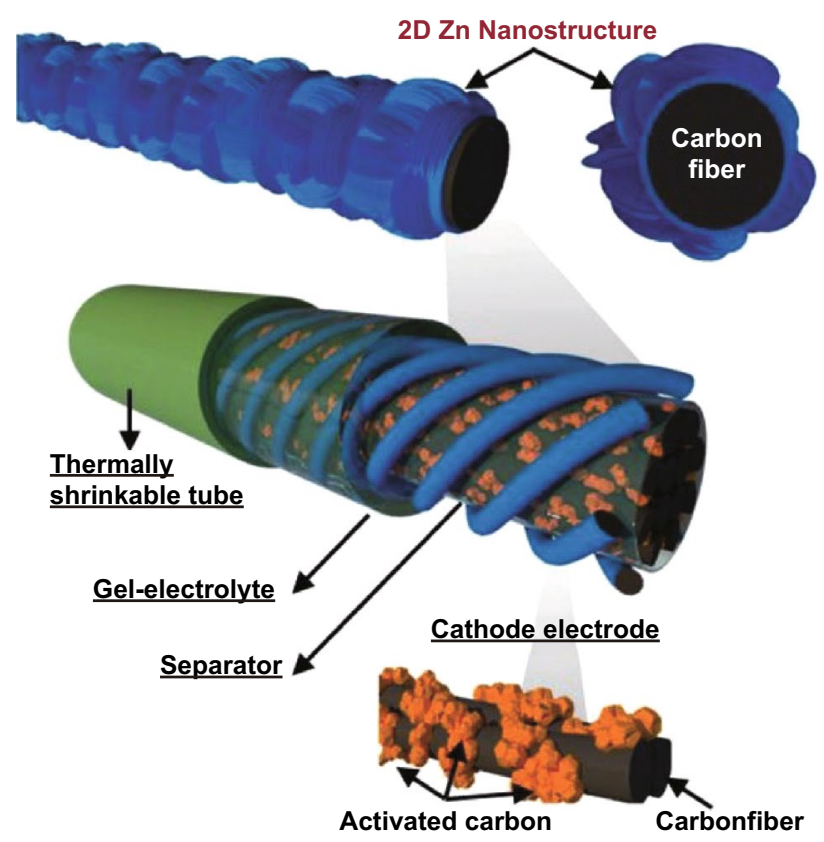

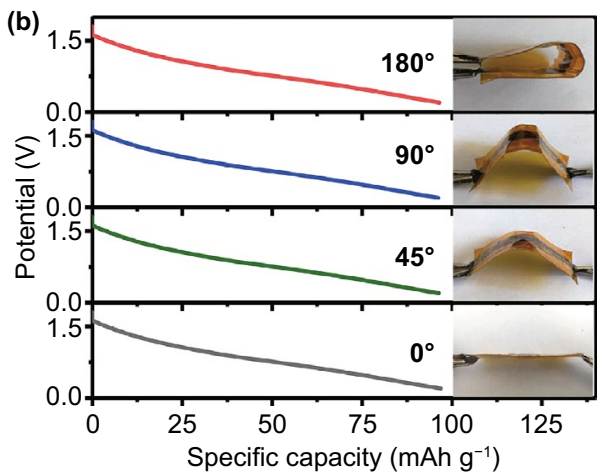

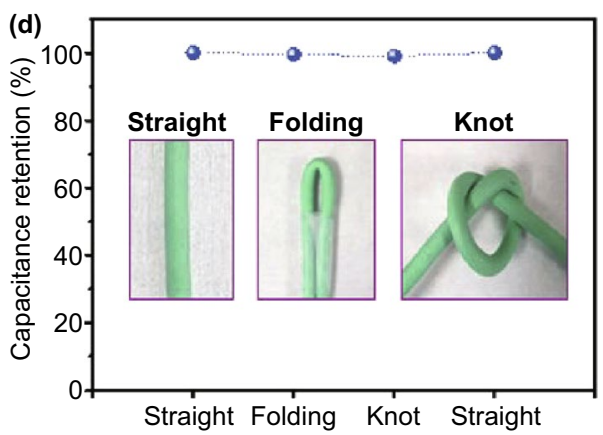

(e)

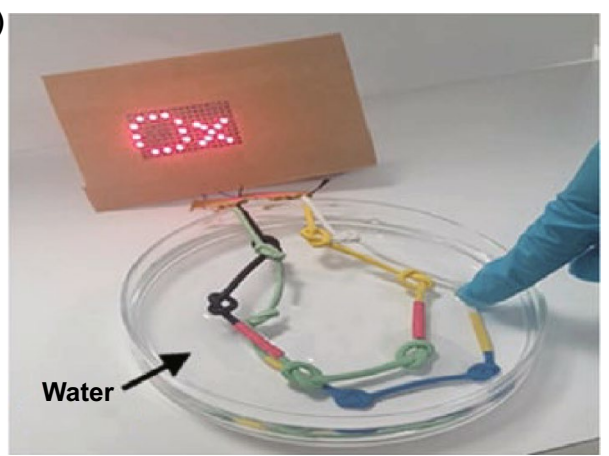

Fig. 13 a Schematics of sheet-type flexible ZHSCs. b Discharge curves under different deformation conditions. Reproduced with permission from Ref. [83]. Copyright 2019, Elsevier. c Schematic of fiber-type flexible ZHSCs. d Capacity retention under the condition of straight, folded and knotted. e LED powered by connected fiber-type ZHSCs in water. Reproduced with permission from Ref. [53]. Copyright 2020, Wiley$\mathrm{VCH}$ 
to form an optimal configuration and make full use of the limited space of ZmSCs.

\section{Flexible Zn-Ion Hybrid Supercapacitors}

Emerging flexible electronic devices are well developed in recent years, such as smart textiles, soft robotics and biosensors [220-223]. Further paradigm shift should be the seamless integration of flexible devices with flexible ESSs. Among the Zn-ion energy storage systems, flexible ZIBs have been widely investigated owing to various features of high flexibility, superb mechanical strength, excellent stability and safety [86, 224]. The application of gel and even solid-state electrolyte make the flexible ZIBs as a promising candidate for the flexible energy storage systems. However, most reported flexible ZIBs suffer from the short longevity (about 100 to 1000 cycles) and low power density [225-227]. Under this circumstance, the discovery of ZHSCs aroused the interests of flexible ZHSCs principally due to a range of attractive features, such as ultra-long cycling lifetime, costeffectiveness, good safety and eco-friendliness. By far, two typical types (sheet-type and fiber-type) of flexible ZHSCs have been reported, to some extent, the shapes of the flexible ZHSCs can determine the corresponding conditions of practical application, for instance, wearable electronic devices usually demand high areal capacity flexible ZHSCs, which can minimize the thickness of the devices and create a better wearing feeling. When applied to the wireless headset, the 1D fiber-shape ZHSCs are preferred due to the 3D flexibility of the linear structure. In the following section, we amply summarized the components and structure of the two typical types of flexible ZHSCs.

\subsection{Sheet-Type ZHSCs}

Sheet-type flexible ZHSCs focus on promoting the areal capacity depends on sheet-type flexible electrodes. Up to now, many outstanding investigations have been reported [99, 132, 154, 198]. For one, Lu et al. built a quasi-solidstate $\mathrm{Zn} / / \mathrm{ZnSO}_{4}$ (gel)//BN-LDC sheet-type flexible $\mathrm{Zn} / / \mathrm{Cap}$ ZHSCs, the schematic illustration is depicted in Fig. 13a where the graphite paper acted as the current collection of LDC cathode, the $\mathrm{Zn}$ foil served as anode and current collector [83]. Moreover, the Whatman filter and 1.0 $\mathrm{M} \mathrm{ZnSO}_{4}$ gel are employed as separator and electrolyte, respectively. Correspondingly, the sheet-type ZHSCs provided a specific capacity of $116.8 \mathrm{mAh} \mathrm{g}^{-1}$ at $0.5 \mathrm{~A} \mathrm{~g}^{-1}$ and further retain $55.4 \mathrm{mAh} \mathrm{g}^{-1}$ at $20 \mathrm{~A} \mathrm{~g}^{-1}$. Additionally, the $\mathrm{Zn} / /$ BN-LDC flexible ZHSCs device can be arbitrarily bent or twisted without deteriorating the discharge curve and capacity (97 $\mathrm{mAh} \mathrm{g}^{-1}$ at $1 \mathrm{~A} \mathrm{~g}^{-1}$, Fig. 13b). Typically, the serieswound ZHSCs are employed in a practical application and lightened a blue light-emitting diode (LED) for $~ 30 \mathrm{~min}$ (inset of Fig. 13a). Notably, the flexible ZHSCs exhibited a good cycling performance of sustaining $81.3 \%$ of the initial capacitance and nearly $100 \%$ CE after 6500 cycles. The $\mathrm{Zn}$ foil presented no evident dendrites and the $\mathrm{BN}$ LDC retains 2D layered structure after a long-term cycling test. For another, the sheet-type flexible ZHSC of Cap// ZBC ZHSCs was reported by Mai group [34]. The flexible $\mathrm{ACC} / / \mathrm{Zn}_{x} \mathrm{MnO}_{2} \mathrm{ZHSCs}$ can afford a prominent capacity of $1446.6 \mathrm{mF} \mathrm{cm}^{-2}$ and ultra-high areal energy density of 803.6 $\mu \mathrm{Wh} \mathrm{cm}{ }^{-2}$. Considering the above results, the 2D sheet-type flexible ZHSCs show wild application prospects for plane electronic devices due to the splendid areal electrochemical performance, good mechanical flexibility and high safety, which can be a promising candidate for future flexible ESSs.

\subsection{Fiber-Type ZHSCs}

Compared with usual sheet-type flexible ZHSCs, fibertype flexible ZHSCs are less discussed, however, the fibertype ZHSCs based on one-dimensional (1D) fiber-shape electrodes can perform better mechanical flexibility than 2D sheet-type ZHSCs. Typically, the flexibility of fibertype ZHSCs is based on the flexible fiber substrate such as carbon fiber (CF), graphite fiber and CNT [120], while the sheet-type ZHSCs achieve flexibility by the intrinsic flexible electrodes like Zn foil and CC [34, 83]. An et al. prepared an outstanding 1D fiber-type ZHSCs and the explicit schematic illustration is depicted in Fig. 13c [53]. Structurally, the fiber-type ZHSCs are composed of 2D-Zn@CF anode, AC@CF cathode, separator, gel electrolyte and thermally shrinkable tube. The AC@CF cathode is enclosed by cellulose papers which act as the separator. Subsequently, the AC@CF cathode is weaved by the 2D-Zn@CF anode and the $1.0 \mathrm{M} \mathrm{ZnSO}_{4} / \mathrm{PVA}$ gel electrolyte is coated on the fiber-type ZHSCs, then covered with thermally shrinkable tubes to protect fiber-type 


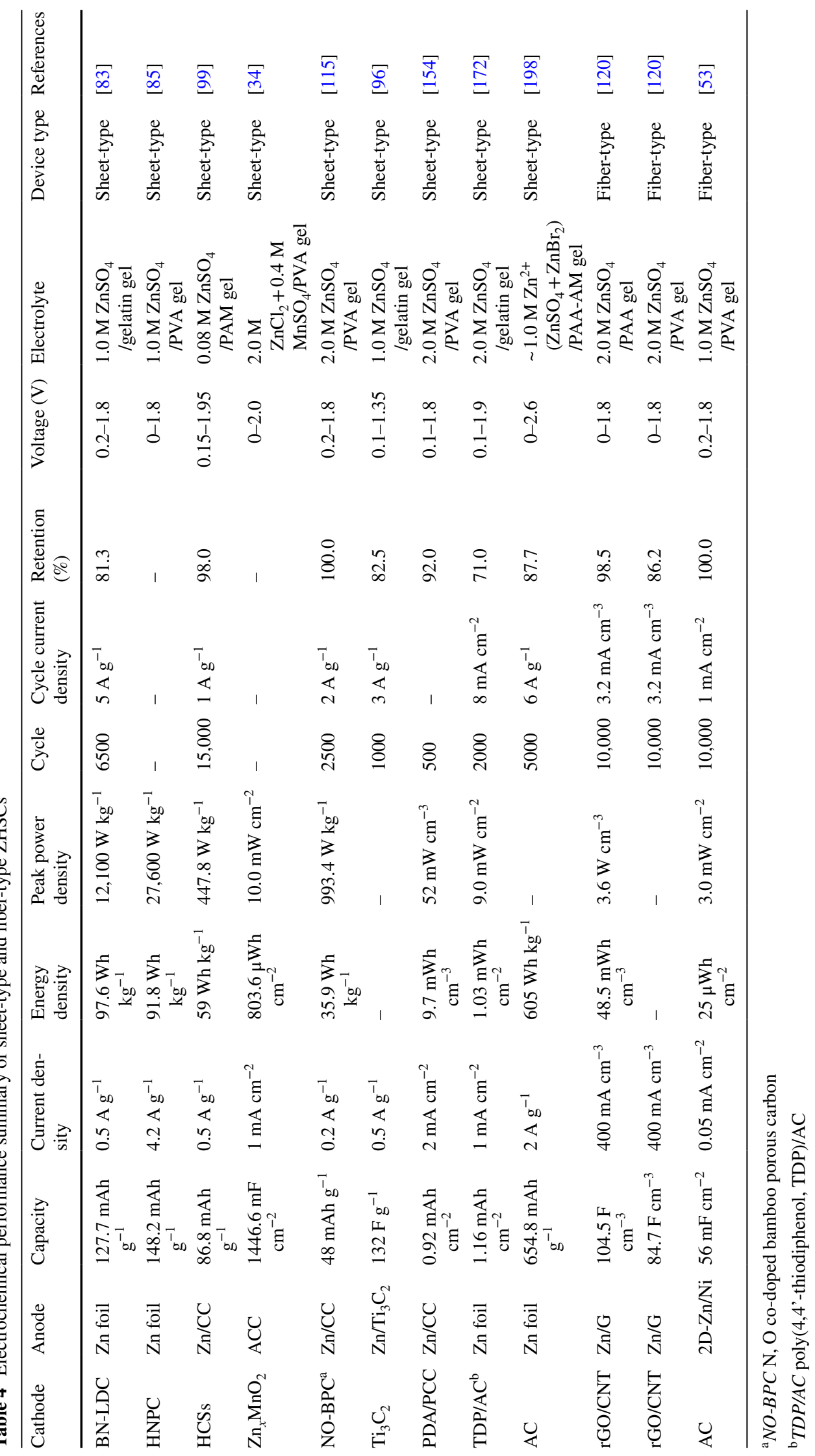


ZHSCs from external influence. The length and diameter of the fiber-type ZHSCs are $15 \mathrm{~cm}$ and $8-9 \mu \mathrm{m}$, respectively. Based on the well-designed structure, the fiber-type ZHSCs provided a high capacity of $56-24 \mathrm{mF} \mathrm{cm}^{-2}$ at a current density of $0.05-3.0 \mathrm{~mA} \mathrm{~cm}{ }^{-2}$. Moreover, the energy density of $25 \mu \mathrm{Wh} \mathrm{cm}{ }^{-2}$ is realized by fiber-type ZHSCs at a high power density of $50 \mu \mathrm{W} \mathrm{cm}{ }^{-2}$. As shown in Fig. 13d, the fiber-type ZHSCs can keep the initial energy storage performance under the condition of folding and knotting, demonstrating superb mechanical flexibility of fiber-type ZHSCs. To evaluate the waterproof capability of the fiber-type ZHSCs, the LEDs were powered by multiple fiber-type ZHSCs which are strongly knotted and under water immersion (Fig. 13e). Furthermore, the cycling lifetime of fiber-type ZHSCs reached 10,000 cycles, and the capacity presented no evident decay, which is superior to many reported flexible supercapacitors [228-233]. Here, we summarized the electrochemical performance of two types of flexible ZHSCs in Table 4.

According to the above reviews and our own experience, the keys to fabricating a flexible ZHSC are gel electrolyte and packaging technology. During the fabrication processes, the flexible devices tend to short-circuit due to the fast diffusion rate and electron transport, which can lead to a high defect rate. A great number of electrolytes are added to achieve excellent contact between electrodes and electrolytes, but the flow of the quasi-solid-state is uncontrollable. In this regard, short-circuit can quickly occur when the separator fails due to the leakage of electrolyte or some manufactural defects of the separator. In addition, the flexible ZHSCs can maintain stable charging/discharging processes at low current density, however, turn to short-circuits at high current density. Once the short circuits occur, the flexible ZHSCs devices cannot return to normal even at low current density. Hence, a relatively low concentration electrolyte and suited polymer gel may be optimal for flexible ZHSCs, Chen et al. employed a low concentration of $0.08 \mathrm{M} \mathrm{ZnSO}_{4} / \mathrm{PAM}$ gel electrolyte for ZHSCs, the ZHSCs presented capacity of $86.8 \mathrm{mAh} \mathrm{g}^{-1}$ and 15,000 cycles with $98 \%$ capacity retention [99]. Additionally, outstanding packaging technology can vastly decrease the probability of malfunction, for instance, the fiber-type flexible ZHSCs are preferable to sheet-type flexible ZHSCs because the electrodes are roundly covered by a separator to avoid the leakage of electrolytes, which suppresses unexpected short circuits [53]. Based on the splendid properties of ZHSCs, the flexible ZHSCs also showed unprecedented electrochemical performance among various flexible ESSs. Even though flexible ZHSC is still in its infancy and more efforts should be devoted to improving the reliability of flexible ZHSC devices, it is believed that flexible ZHSCs can be qualified for high-performance flexible electronic devices.

\section{Summary and Outlook}

In this review, we have expounded the design theory by summarizing recent research progresses of ZHSCs and classifying them into two electrode configurations of Zn//Cap ZHSCs and Cap//ZBC ZHSCs. All in all, these ZHSCs integrate various advantages of conventional supercapacitors and ZIBs, presenting high energy density, high power density, splendid rate performance and excellent cycling longevity. In addition to the aqueous ZHSCs, this review summarizes practical applications of ZHSCs, such as high-performance ZmSCs and flexible sheet/fibertype ZHSCs, which indicate the promising prospects of ZHSCs for various applications. Although many improvements have been achieved in the ZHSCs field, ZHSCs system is still in its infancy compared to some mature ESSs. Therefore, we believe that it should pay more attention to the following key points for the prospective research of ZHSCs:

(1) Better understandings of electrode materials are required

When simply employing commercial $\mathrm{Zn}$ foil and AC materials as electrodes, the electrochemical performances of $\mathrm{Zn} / / \mathrm{Cap}$ ZHSCs are relatively limited. In this regard, the development of specific electrodes for ZHSCs is quite needed, where the $\mathrm{Zn}$ anode should be hierarchically designed including high-performance background $\mathrm{Zn}$ and surface $\mathrm{Zn}$ while more strategies should be applied to promote the combination of carbon-based materials and some novel materials, which possess fast and pseudocapacitive $\mathrm{Zn}$-ion storage mechanisms. In a word, preparing the battery-type electrodes in the methodology of Cap electrodes, while the Cap electrodes the methodology of battery-type, then the trade-off between energy density and cycling performance can be well-balanced. 
(2) Attempt of mature ZBC for ZHSCs

Even though the Cap//ZBC ZHSC is still in its infancy, the great designability and unique properties like high voltage window and $\mathrm{Zn}$-dendrite-free of Cap// ZBC ZHSCs make it a promising ESS in the future. To date, the $\mathrm{ZBC}$ are suffering from one or more of the following deficiencies: difficulty in precise control, inferior rate performance, weak cycling performance, low energy density. Designing advanced cathodes (e.g., Mn, V, PBA, Mo, Co-based cathodes) with superb structural stability, large specific capacity and suitable $\mathrm{Zn}$-ion storage mechanisms can greatly promote the electrochemical performance of Cap//ZBC ZHSCs. Some splendid strategies for cathode materials in the ZIBs can also be applied in ZHSCs and further summarize the ZHSCs-proper design strategies of ZBC. We strongly believe that the Cap//ZBC ZHSCs deserve more investigations.

(3) Confirm the by-products mechanisms and find solutions Although the precipitation/dissolution processes of $\mathrm{Zn}_{4} \mathrm{SO}_{4}(\mathrm{OH})_{6} \cdot \mathrm{nH}_{2} \mathrm{O}$ or $\mathrm{ZnSO}_{3} \cdot 2.5\left(\mathrm{H}_{2} \mathrm{O}\right)$ by-products have been analyzed at length in this review, more studies are needed to confirm the conclusions and find feasible ways out to prevent the irreversible precipitation/ dissolution processes of both electrodes in ZHSCs. It is believed that the novel electrolytes like $\mathrm{Zn}\left(\mathrm{CH}_{3} \mathrm{COO}\right)_{2}$, $\mathrm{Zn}\left(\mathrm{ClO}_{4}\right)_{2}$ and $\mathrm{ZnCl}_{2}$ will be the key for achieving high-performance and by-product-free ZHSCs.

(4) Appropriate additives may optimize energy storage behaviors

According to the reported works, the additive electrolytes can tremendously promote the electrochemical performance of ZHSCs. For example, the Mn-ion additive can restrain the dissolution of manganese-based materials, the $\mathrm{Br}$-ion additive can increase the voltage window to $2.6 \mathrm{~V}$ and enable a controllable $\mathrm{Zn}$ deposition behavior. A few additives may vastly boost the electrochemical performance of ZHSCs, thus, more cation and anion additive electrolytes should be tried and introduced into the normal Zn-ion salt electrolytes to achieve high-performance ZHSCs with special properties.

(5) Attempts of 3D materials and establishment of mechanical standards in ZmSCs

The reported in-plane $\mathrm{ZmSCs}$ have gained much attention due to their efficient energy storage capacity, which is much more than the traditional mSCs. However, the electrodes of ZmSCs are based on thin-film electrodes and the materials are still conventional. The 3D porous Cap cathodes should be introduced for highperformance $\mathrm{ZmSCs}$ and some efforts are necessary for investigating the Cap//ZBC ZmSCs. Moreover, the "flexibility" of ZmSCs is still a vague definition, it is urgently needed to build a flexible mechanical standard for ZmSC, which can serve as the guidance for future assessment.

(6) Improve the stability of flexible ZHSCs devices

Same as the ZHSCs, the flexible ZHSCs are new products in flexible ESSs. With the excellent electrochemical performance and safe aqueous gel electrolytes, the flexible ZHSCs devices are a promising candidate for new generation flexible ESSs. The fiber-type ZHSCs can be knitted into fabrics, offering a viable solution toward wearable ESSs. However, flexible ZHSCs presented unstable charging/discharging behaviors at high current density, and the synthetic success rate is much lower than ZIBs devices due to the fast diffusion rate and electron transport. Thus, the explorations of flexible ZHSCs are far from satisfied, where the studies of low-concentration gel electrolyte and precise packaging technology may be the keys.

Although tremendous efforts have been made, it should be emphasized that the ZHSCs still need a long way to be applied in commercial applications. We would rather regard this review as an opening remark than a concluding remark. We believe the ZHSCs will break some current bottlenecks in the fields of energy storage and eventually, realizing better $\mathrm{Zn}$-ion storage systems with balanced electrochemical performances.

Acknowledgements This work was supported by National Natural Science Foundation (U1802254, U20A20168, 61874065, 51861145202) of China, the National Key R\&D Program (2018YFC2001202), Zhejiang University of Technology's Research Start-up Foundation (2021125010629), Xiangshun Geng thanks for the support from Shuimu Tsinghua Scholar Program.

Funding Open access funding provided by Shanghai Jiao Tong University.

Open Access This article is licensed under a Creative Commons Attribution 4.0 International License, which permits use, sharing, adaptation, distribution and reproduction in any medium or format, as long as you give appropriate credit to the original author(s) and the source, provide a link to the Creative Commons licence, and indicate if changes were made. The images or other third party material in this article are included in the article's Creative Commons licence, unless indicated otherwise in a credit line to the material. If material is not included in the article's Creative Commons licence and your intended use is not permitted by statutory regulation or exceeds the permitted use, you will need to obtain permission directly from the copyright holder. To view a copy of this licence, visit http://creativecommons.org/licenses/by/4.0/. 


\section{References}

1. B. Dunn, H. Kamath, J.M. Tarascon, Electrical energy storage for the grid: a battery of choices. Science 334(6058), 928-935 (2011). https://doi.org/10.1126/science.1212741

2. P. Simon, Y. Gogotsi, Materials for electrochemical capacitors. Nat. Mater. 7, 845-854 (2008). https://doi.org/10.1038/ nmat2297

3. Y. Huang, Y. Wang, C. Tang, J. Wang, Q. Zhang et al., Atomic modulation and structure design of carbons for bifunctional electrocatalysis in metal-air batteries. Adv. Mater. 31(13), 1803800 (2019). https://doi.org/10.1002/adma.201803800

4. Z.P. Cano, D. Banham, S. Ye, A. Hintennach, K. Lu et al., Batteries and fuel cells for emerging electric vehicle markets. Nat. Energy 3(4), 279-289 (2018). https://doi.org/10.1038/ s41560-018-0108-1

5. M. Borghei, J. Lehtonen, L. Liu, O.J. Rojas, Advanced biomass-derived electrocatalysts for the oxygen reduction reaction. Adv. Mater. 30(24), 1703691 (2018). https://doi.org/10. 1002/adma.201703691

6. C. Yang, J. Chen, X. Ji, T.P. Pollard, X. Lu et al., Aqueous Li-ion battery enabled by halogen conversion-intercalation chemistry in graphite. Nature 569(7755), 245-250 (2019). https://doi.org/10.1038/s41586-019-1175-6

7. F. Wan, Y. Zhang, L. Zhang, D. Liu, C. Wang et al., Reversible oxygen redox chemistry in aqueous zinc-ion batteries. Angew. Chem. Int. Ed. 58(21), 7062-7067 (2019). https:// doi.org/10.1002/anie.201902679

8. J. Qian, C. Wu, Y. Cao, Z. Ma, Y. Huang et al., Prussian blue cathode materials for sodium-ion batteries and other ion batteries. Adv. Energy Mater. 8(17), 1702619 (2018). https://doi. org/10.1002/aenm.201702619

9. L. Jiang, Y. Lu, C. Zhao, L. Liu, J. Zhang et al., Building aqueous K-ion batteries for energy storage. Nat. Energy 4(6), 495-503 (2019). https://doi.org/10.1038/s41560-019-0388-0

10. F. Wu, H. Yang, Y. Bai, C. Wu, Paving the path toward reliable cathode materials for aluminum-ion batteries. Adv. Mater. 31(16), 1806510 (2019). https://doi.org/10.1002/adma.20180 6510

11. C. Zhang, V. Nicolosi, Graphene and MXene-based transparent conductive electrodes and supercapacitors. Energy Storage Mater. 16, 102-125 (2019). https://doi.org/10.1016/j. ensm.2018.05.003

12. T. Lv, M. Liu, D. Zhu, L. Gan, T. Chen, Nanocarbon-based materials for flexible all-solid-state supercapacitors. Adv. Mater. 30(17), 1705489 (2018). https://doi.org/10.1002/ adma.201705489

13. Y. Da, J. Liu, L. Zhou, X. Zhu, X. Chen et al., Engineering 2D architectures toward high-performance micro-supercapacitors. Adv. Mater. 31(1), 1802793 (2019). https://doi.org/10. 1002/adma.201802793

14. Q. Ren, H. Wang, X. Lu, Y. Tong, G. Li, Recent progress on MOF-derived heteroatom-doped carbon-based electrocatalysts for oxygen reduction reaction. Adv. Sci. 5(3), 1700515 (2018). https://doi.org/10.1002/advs.201700515
15. H. Qiu, P. Du, K. Hu, J. Gao, H. Li et al., Metal and nonmetal codoped 3D nanoporous graphene for efficient bifunctional electrocatalysis and rechargeable Zn-air batteries. Adv. Mater. 31(19), 1900843 (2019). https://doi.org/10.1002/adma.20190 0843

16. J. Li, S. Chen, N. Yang, M. Deng, S. Ibraheem et al., Ultrahigh-loading zinc single-atom catalyst for highly efficient oxygen reduction in both acidic and alkaline media. Angew. Chem. Int. Ed. 58(21), 7035-7039 (2019). https://doi.org/10. 1002/anie.201902109

17. Y. Yang, Y. Tang, G. Fang, L. Shan, J. Guo et al., Li ${ }^{+}$intercalated $\mathrm{V}_{2} \mathrm{O}_{5} \cdot \mathrm{nH}_{2} \mathrm{O}$ with enlarged layer spacing and fast ion diffusion as an aqueous zinc-ion battery cathode. Energy Environ. Sci. 11(11), 3157-3162 (2018). https://doi.org/10. 1039/c8ee01651h

18. C. Liu, Z. Neale, J. Zheng, X. Jia, J. Huang et al., Expanded hydrated vanadate for high-performance aqueous zinc-ion batteries. Energy Environ. Sci. 12(7), 2273-2285 (2019). https://doi.org/10.1039/c9ee00956f

19. W. Chen, T. Lei, C. Wu, M. Deng, C. Gong et al., Designing safe electrolyte systems for a high-stability lithium-sulfur battery. Adv. Energy Mater. 8(10), 1702348 (2018). https://doi. org/10.1002/aenm.201702348

20. B. Li, J. Zheng, H. Zhang, L. Jin, D. Yang et al., Electrode materials, electrolytes, and challenges in nonaqueous lithiumion capacitors. Adv. Mater. 30(17), 1705670 (2018). https:// doi.org/10.1002/adma.201705670

21. C. Lethien, J.L. Bideau, T. Brousse, Challenges and prospects of 3D micro-supercapacitors for powering the internet of things. Energy Environ. Sci. 12(1), 96-115 (2019). https://doi.org/10.1039/c8ee02029a

22. W. Guo, C. Yu, S. Li, Z. Wang, J. Yu et al., Strategies and insights towards the intrinsic capacitive properties of $\mathrm{MnO}_{2}$ for supercapacitors: challenges and perspectives. Nano Energy 57, 459-472 (2019). https://doi.org/10.1016/j. nanoen.2018.12.015

23. J. Jiang, Y. Li, J. Liu, X. Huang, C. Yuan et al., Recent advances in metal oxide-based electrode architecture design for electrochemical energy storage. Adv. Mater. 24(38), 5166-5180 (2012). https://doi.org/10.1002/adma.20120 2146

24. J.W. Choi, D. Aurbach, Promise and reality of post-lithiumion batteries with high energy densities. Nat. Rev. Mater. 1(4), 16013 (2016). https://doi.org/10.1038/natrevmats. 2016.13

25. L.L. Zhang, X.S. Zhao, Carbon-based materials as supercapacitor electrodes. Chem. Soc. Rev. 38(9), 2520-2531 (2009). https://doi.org/10.1039/b813846j

26. M. Yu, X. Feng, Thin-film electrode-based supercapacitors. Joule 3(2), 338-360 (2019). https://doi.org/10.1016/j.joule. 2018.12.012

27. H. Jiang, P.S. Lee, C. Li, 3D carbon based nanostructures for advanced supercapacitors. Energy Environ. Sci. 6(1), 41-53 (2013). https://doi.org/10.1039/c2ee23284g

28. J. Huang, B.G. Sumpter, V. Meunier, Theoretical model for nanoporous carbon supercapacitors. Angew. Chem. Int. Ed. 
47(3), 520-524 (2008). https://doi.org/10.1002/anie.20070 3864

29. B. Kang, G. Ceder, Battery materials for ultrafast charging and discharging. Nature 458(7235), 190-193 (2009). https://doi.org/10.1038/nature07853

30. M.R. Lukatskaya, B. Dunn, Y. Gogotsi, Multidimensional materials and device architectures for future hybrid energy storage. Nat. Commun. 7, 12647 (2016). https://doi.org/10. 1038/ncomms 12647

31. L. Dong, X. Ma, Y. Li, L. Zhao, W. Liu et al., Extremely safe, high-rate and ultralong-life zinc-ion hybrid supercapacitors. Energy Storage Mater. 13, 96-102 (2018). https:// doi.org/10.1016/j.ensm.2018.01.003

32. P. Zhang, Y. Li, G. Wang, F. Wang, S. Yang et al., Zn-ion hybrid micro-supercapacitors with ultrahigh areal energy density and long-term durability. Adv. Mater. 31(3), 1806005 (2019). https://doi.org/10.1002/adma.201806005

33. W. Zuo, R. Li, C. Zhou, Y. Li, J. Xia et al., Battery-supercapacitor hybrid devices: recent progress and future prospects. Adv. Sci. 4(7), 1600539 (2017). https://doi.org/10. 1002/advs.201600539

34. Q. Chen, J. Jin, Z. Kou, C. Liao, Z. Liu et al., $\mathrm{Zn}^{2+}$ preintercalation stabilizes the tunnel structure of $\mathrm{MnO}_{2}$ nanowires and enables zinc-ion hybrid supercapacitor of battery-level energy density. Small 16(14), 2000091 (2020). https://doi.org/10.1002/smll.202000091

35. L. Dong, W. Yang, W. Yang, Y. Li, W. Wu et al., Multivalent metal ion hybrid capacitors: a review with a focus on zinc-ion hybrid capacitors. J. Mater. Chem. A 7(23), 13810-13832 (2019). https://doi.org/10.1039/c9ta02678a

36. B.D. Boruah, Roadmap of in-plane electrochemical capacitors and their advanced integrated systems. Energy Storage Mater. 21, 219-239 (2019). https://doi.org/10.1016/j.ensm. 2019.06.012

37. W. Fu, E. Zhao, R. Ma, Z. Sun, Y. Yang et al., Anatase $\mathrm{TiO}_{2}$ confined in carbon nanopores for high-energy Li-ion hybrid supercapacitors operating at high rates and subzero temperatures. Adv. Energy Mater. 10(2), 1902993 (2020). https://doi.org/10.1002/aenm.201902993

38. D. Tie, S. Huang, J. Wang, J. Ma, J. Zhang et al., Hybrid energy storage devices: advanced electrode materials and matching principles. Energy Storage Mater. 21, 22-40 (2019). https://doi.org/10.1016/j.ensm.2018.12.018

39. A. Muzaffar, M.B. Ahamed, K. Deshmukh, J. Thirumalai, A review on recent advances in hybrid supercapacitors: design, fabrication and applications. Renew. Sust. Energ. Rev. 101, 123-145 (2019). https://doi.org/10.1016/j.rser. 2018.10.026

40. H. Wang, W. Ye, Y. Yang, Y. Zhong, Y. Hu, Zn-ion hybrid supercapacitors: achievements, challenges and future perspectives. Nano Energy 85, 105942 (2021). https://doi.org/ 10.1016/j.nanoen.2021.105942

41. E. Kim, H. Kim, B.J. Park, Y.H. Han, J.H. Park et al., Etching-assisted crumpled graphene wrapped spiky iron oxide particles for high-performance Li-ion hybrid supercapacitor.
Small 14(16), 1704209 (2018). https://doi.org/10.1002/smll. 201704209

42. Z. Hu, S. Sayed, T. Jiang, X. Zhu, C. Lu et al., Self-assembled binary organic granules with multiple lithium uptake mechanisms toward high-energy flexible lithium-ion hybrid supercapacitors. Adv. Energy Mater. 8(30), 1802273 (2018). https://doi.org/10.1002/aenm.201802273

43. B. Deng, T. Lei, W. Zhu, L. Xiao, J. Liu, In-plane assembled orthorhombic $\mathrm{Nb}_{2} \mathrm{O}_{5}$ nanorod films with high-rate $\mathrm{Li}^{+}$intercalation for high-performance flexible Li-ion capacitors. Adv. Funct. Mater. 28(1), 1704330 (2018). https://doi.org/10.1002/ adfm.201704330

44. L. Que, F. Yu, Z. Wang, D. Gu, Pseudocapacitance of $\mathrm{TiO}_{2-\mathrm{x}} /$ CNT anodes for high-performance quasi-solid-state $\mathrm{Li}$-ion and Na-ion capacitors. Small 14(17), 1704508 (2018). https:// doi.org/10.1002/smll.201704508

45. Y. Zhu, L. Yang, J. Sheng, Y. Chen, H. Gu et al., Fast sodium storage in $\mathrm{TiO}_{2} @ \mathrm{CNT} @ \mathrm{C}$ nanorods for high-performance Na-ion capacitors. Adv. Energy Mater. 7(22), 1701222 (2017). https://doi.org/10.1002/aenm.201701222

46. H. Wang, C. Zhu, D. Chao, Q. Yan, H.J. Fan, Nonaqueous hybrid lithium-ion and sodium-ion capacitors. Adv. Mater. 29(46), 1702093 (2017). https://doi.org/10.1002/adma.20170 2093

47. H. Wang, D. Xu, G. Jia, Z. Mao, Y. Gong et al., Integration of flexibility, cyclability and high-capacity into one electrode for sodium-ion hybrid capacitors with low self-discharge rate. Energy Storage Mater. 25, 114-123 (2020). https://doi.org/ 10.1016/j.ensm.2019.10.024

48. Z. Tong, S. Liu, Y. Zhou, J. Zhao, Y. Wu et al., Rapid redox kinetics in uniform sandwich-structured mesoporous $\mathrm{Nb}_{2} \mathrm{O}_{5} /$ graphene/mesoporous $\mathrm{Nb}_{2} \mathrm{O}_{5}$ nanosheets for high-performance sodium-ion supercapacitors. Energy Storage Mater. 13, 223-232 (2018). https://doi.org/10.1016/j.ensm.2017.12. 005

49. P. Zhang, L. Wang, F. Wang, D. Tan, G. Wang et al., A nonaqueous Na-ion hybrid micro-supercapacitor with wide potential window and ultrahigh areal energy density. Batteries Supercaps 2(11), 918-923 (2019). https://doi.org/10.1002/ batt.201900079

50. Y. Yi, Z. Sun, C. Li, Z. Tian, C. Lu et al., Designing 3D biomorphic nitrogen-doped $\mathrm{MoSe}_{2} /$ graphene composites toward high-performance potassium-ion capacitors. Adv. Funct. Mater. 30(4), 1903878 (2020). https://doi.org/10.1002/ adfm. 201903878

51. J. Ruan, F. Mo, Z. Chen, M. Liu, S. Zheng et al., Rational construction of nitrogen-doped hierarchical dual-carbon for advanced potassium-ion hybrid capacitors. Adv. Energy Mater. 10(15), 1904045 (2020). https://doi.org/10.1002/ aenm.201904045

52. Y. Cui, W. Liu, W. Feng, Y. Zhang, Y. Du et al., Controlled design of well-dispersed ultrathin $\mathrm{MoS}_{2}$ nanosheets inside hollow carbon skeleton: toward fast potassium storage by constructing spacious "houses" for K ions. Adv. Funct. Mater. 30(10), 1908755 (2020). https://doi.org/10.1002/adfm.20190 8755 
53. G. An, J. Hong, S. Pak, Y. Cho, S. Lee et al., 2D metal $\mathrm{Zn}$ nanostructure electrodes for high-performance $\mathrm{Zn}$ ion supercapacitors. Adv. Energy Mater. 10(3), 1902981 (2020). https://doi.org/10.1002/aenm.201902981

54. Y. Zhang, Z. Zhang, Y. Tang, D. Jia, Y. Huang et al., LiFePO particles embedded in fast bifunctional conductor rGO\&C@ $\mathrm{Li}_{3} \mathrm{~V}_{2}\left(\mathrm{PO}_{4}\right)_{3}$ nanosheets as cathodes for high-performance Li-ion hybrid capacitors. Adv. Funct. Mater. 29(17), 1807895 (2019). https://doi.org/10.1002/adfm.201807895

55. R. Wang, P. Liu, J. Lang, L. Zhang, X. Yan, Coupling effect between ultra-small $\mathrm{Mn}_{3} \mathrm{O}_{4}$ nanoparticles and porous carbon microrods for hybrid supercapacitors. Energy Storage Mater. 6, 53-60 (2017). https://doi.org/10.1016/j.ensm.2016.10.002

56. H. Liu, L. Liao, Y. Lu, Q. Li, High energy density aqueous Liion flow capacitor. Adv. Energy Mater. 7(1), 1601248 (2017). https://doi.org/10.1002/aenm.201601248

57. J. Li, L. An, H. Li, J. Sun, C. Shuck et al., Tunable stable operating potential window for high-voltage aqueous supercapacitors. Nano Energy 63, 103848 (2019). https://doi.org/ 10.1016/j.nanoen.2019.06.044

58. Y. Li, H. Wang, L. Wang, Z. Mao, R. Wang et al., Mesoporeinduced ultrafast $\mathrm{Na}^{+}$-storage in $\mathrm{T}-\mathrm{Nb}_{2} \mathrm{O}_{5}$ /carbon nanofiber films toward flexible high-power Na-ion capacitors. Small 15(9), 1804539 (2019). https://doi.org/10.1002/smll.20180 4539

59. H. Huang, D. Kundu, R. Yan, E. Tervoort, X. Chen et al., Fast Na-ion intercalation in zinc vanadate for high-performance Na-ion hybrid capacitor. Adv. Energy Mater. 8(35), 1802800 (2018). https://doi.org/10.1002/aenm.201802800

60. S. Dong, Y. Xu, L. Wu, H. Dou, X. Zhang, Surface-functionalized graphene-based quasi-solid-state Na-ion hybrid capacitors with excellent performance. Energy Storage Mater. 11, 8-15 (2018). https://doi.org/10.1016/j.ensm.2017.09.006

61. C. An, Y. Yuan, B. Zhang, L. Tang, B. Xiao et al., Graphene wrapped $\mathrm{FeSe}_{2}$ nano-microspheres with high pseudocapacitive contribution for enhanced Na-ion storage. Adv. Energy Mater. 9(18), 1900356 (2019). https://doi.org/10.1002/aenm. 201900356

62. K.V. Nielson, T.L. Liu, Dawn of calcium batteries. Angew. Chem. Int. Ed. 59(9), 3368-3370 (2020). https://doi.org/10. 1002/anie.201913465

63. F. Wu, J. Maier, Y. Yu, Guidelines and trends for next-generation rechargeable lithium and lithium-ion batteries. Chem. Soc. Rev. 49(5), 1569-1614 (2020). https://doi.org/10.1039/ c7cs00863e

64. M.F. Lagadec, R. Zahn, V. Wood, Characterization and performance evaluation of lithium-ion battery separators. Nat. Energy 4(1), 16-25 (2019). https://doi.org/10.1038/ s41560-018-0295-9

65. J. Liu, Z. Bao, Y. Cui, E.J. Dufek, J.B. Goodenough et al., Pathways for practical high-energy long-cycling lithium metal batteries. Nat. Energy 4(3), 180-186 (2019). https:// doi.org/10.1038/s41560-019-0338-x

66. D. Chao, H.J. Fan, Intercalation pseudocapacitive behavior powers aqueous batteries. Chem 5(6), 1359-1361 (2019). https://doi.org/10.1016/j.chempr.2019.05.020
67. P. He, Q. Chen, M. Yan, X. Xu, L. Zhou et al., Building better zinc-ion batteries: a materials perspective. EnergyChem 1(3), 100022 (2019). https://doi.org/10.1016/j.enchem.2019. 100022

68. S.S. Shinde, C.H. Lee, J.Y. Jung, N.K. Wagh, S.H. Kim et al., Unveiling dual-linkage 3D hexaiminobenzene metal-organic frameworks towards long-lasting advanced reversible $\mathrm{Zn}$ air batteries. Energy Environ. Sci. 12(2), 727-738 (2019). https://doi.org/10.1039/c8ee02679c

69. B. Li, S. Zhang, B. Wang, Z. Xia, C. Tang et al., A porphyrin covalent organic framework cathode for flexible Zn-air batteries. Energy Environ. Sci. 11(7), 1723-1729 (2018). https:// doi.org/10.1039/c8ee00977e

70. Y. Guo, P. Yuan, J. Zhang, Y. Hu, I.S. Amiinu et al., Carbon nanosheets containing discrete $\mathrm{Co}-\mathrm{N}_{\mathrm{x}}-\mathrm{B}_{\mathrm{y}}-\mathrm{C}$ active sites for efficient oxygen electrocatalysis and rechargeable $\mathrm{Zn}$-air batteries. ACS Nano 12(2), 1894-1901 (2018). https://doi.org/ 10.1021/acsnano.7b08721

71. C. Yuan, H.B. Wu, Y. Xie, X.W. Lou, Mixed transition-metal oxides: design, synthesis, and energy-related applications. Angew. Chem. Int. Ed. 53(6), 1488-1504 (2014). https://doi. org/10.1002/anie.201303971

72. J. Liu, C. Guan, C. Zhou, Z. Fan, Q. Ke et al., A flexible quasi-solid-state nickel-zinc battery with high energy and power densities based on 3D electrode design. Adv. Mater. 28(39), 8732-8739 (2016). https://doi.org/10.1002/adma. 201603038

73. T. Ling, T. Zhang, B. Ge, L. Han, L. Zheng et al., Welldispersed nickel- and zinc-tailored electronic structure of a transition metal oxide for highly active alkaline hydrogen evolution reaction. Adv. Mater. 31(16), 1807771 (2019). https://doi.org/10.1002/adma.201807771

74. G. Liang, F. Mo, D. Wang, X. Li, Z. Huang et al., Commencing mild Ag-Zn batteries with long-term stability and ultra-flat voltage platform. Energy Storage Mater. 25, 86-92 (2020). https://doi.org/10.1016/j.ensm.2019.10.028

75. C. Li, Q. Zhang, J. Sun, T. Li, E. Songfeng et al., Highperformance quasi-solid-state flexible aqueous rechargeable Ag-Zn battery based on metal-organic framework-derived Ag nanowires. ACS Energy Lett. 3(11), 2761-2768 (2018). https://doi.org/10.1021/acsenergylett.8b01675

76. S. Berchmans, A.J. Bandodkar, W. Jia, J. Ramirez, Y.S. Meng et al., An epidermal alkaline rechargeable Ag-Zn printable tattoo battery for wearable electronics. J. Mater. Chem. A 2(38), 15788-15795 (2014). https://doi.org/10.1039/c4ta0 $3256 \mathrm{j}$

77. Y. Zhao, Y. Wang, Z. Zhao, J. Zhao, T. Xin et al., Achieving high capacity and long life of aqueous rechargeable zinc battery by using nanoporous-carbon-supported poly(1,5naphthalenediamine) nanorods as cathode. Energy Storage Mater. 28, 64-72 (2020). https://doi.org/10.1016/j.ensm. 2020.03.001

78. Z. Liu, H. Li, B. Shi, Y. Fan, Z.L. Wang et al., Wearable and implantable triboelectric nanogenerators. Adv. Funct. Mater. 29(20), 1808820 (2019). https://doi.org/10.1002/adfm.20180 8820 
79. A. Noori, M.F.E. Kady, M.S. Rahmanifar, R.B. Kaner, M.F. Mousavi, Towards establishing standard performance metrics for batteries, supercapacitors and beyond. Chem. Soc. Rev. 48(5), 1272-1341 (2019). https://doi.org/10.1039/c8cs0 $0581 \mathrm{~h}$

80. L. Liu, Y. Feng, W. Wu, Recent progress in printed flexible solid-state supercapacitors for portable and wearable energy storage. J. Power Sources 410, 69-77 (2019). https://doi.org/ 10.1016/j.jpowsour.2018.11.012

81. S. Yun, Y. Zhang, Q. Xu, J. Liu, Y. Qin, Recent advance in new-generation integrated devices for energy harvesting and storage. Nano Energy 60, 600-619 (2019). https://doi.org/10. 1016/j.nanoen.2019.03.074

82. S. Wu, Y. Chen, T. Jiao, J. Zhou, J. Cheng et al., An aqueous Zn-ion hybrid supercapacitor with high energy density and ultrastability up to 80000 cycles. Adv. Energy Mater. 9(47), 1902915 (2019). https://doi.org/10.1002/aenm.201902915

83. Y. Lu, Z. Li, Z. Bai, H. Mi, C. Ji et al., High energy-power $\mathrm{Zn}$-ion hybrid supercapacitors enabled by layered B/N codoped carbon cathode. Nano Energy 66, 104132 (2019). https://doi.org/10.1016/j.nanoen.2019.104132

84. H. Tang, J. Yao, Y. Zhu, Recent developments and future prospects for zinc-ion hybrid capacitors: a review. Adv. Energy Mater. 11(14), 2003994 (2021). https://doi.org/10. 1002/aenm.202003994

85. H. Zhang, Q. Liu, Y. Fang, C. Teng, X. Liu et al., Boosting Zn-ion energy storage capability of hierarchically porous carbon by promoting chemical adsorption. Adv. Mater. 31(44), 1904948 (2019). https://doi.org/10.1002/adma.201904948

86. P. Yu, Y. Zeng, H. Zhang, M. Yu, Y. Tong et al., Flexible Znion batteries: recent progresses and challenges. Small 15(7), 1804760 (2019). https://doi.org/10.1002/smll.201804760

87. M. Song, H. Tan, D. Chao, H.J. Fan, Recent advances in Znion batteries. Adv. Funct. Mater. 28(41), 1802564 (2018). https://doi.org/10.1002/adfm.201802564

88. D. Wang, W. Zhang, W. Zheng, X. Cui, T. Rojo et al., Towards high-safe lithium metal anodes: suppressing lithium dendrites via tuning surface energy. Adv. Sci. 4(1), 1600168 (2017). https://doi.org/10.1002/advs.201600168

89. Y. Cui, Q. Zhao, X. Wu, Z. Wang, R. Qin et al., Quasi-solid single $\mathrm{Zn}$-ion conductor with high conductivity enabling dendrite-free $\mathrm{Zn}$ metal anode. Energy Storage Mater. 27, 1-8 (2020). https://doi.org/10.1016/j.ensm.2020.01.003

90. Z. Wang, J. Huang, Z. Guo, X. Dong, Y. Liu et al., A metalorganic framework host for highly reversible dendrite-free zinc metal anodes. Joule 3(5), 1289-1300 (2019). https://doi. org/10.1016/j.joule.2019.02.012

91. Z. Fan, J. Jin, C. Li, J. Cai, C. Wei et al., 3D-printed Zn-ion hybrid capacitor enabled by universal divalent cation-gelated additive-free $\mathrm{Ti}_{3} \mathrm{C}_{2}$ MXene ink. ACS Nano 15(2), 3098-3107 (2021). https://doi.org/10.1021/acsnano.0c09646

92. P. Liu, W. Liu, Y. Huang, P. Li, J. Yan et al., Mesoporous hollow carbon spheres boosted, integrated high performance aqueous Zn-ion energy storage. Energy Storage Mater. 25, 858-865 (2020). https://doi.org/10.1016/j.ensm.2019.09.004
93. P. Liu, R. Lv, Y. He, B. Na, B. Wang et al., An integrated, flexible aqueous $\mathrm{Zn}$-ion battery with high energy and power densities. J. Power Sources 410, 137-142 (2019). https://doi. org/10.1016/j.jpowsour.2018.11.017

94. Y. Lu, Y. Wen, F. Huang, T. Zhu, S. Sun et al., Rational design and demonstration of a high-performance flexible $\mathrm{Zn} /$ $\mathrm{V}_{2} \mathrm{O}_{5}$ battery with thin-film electrodes and para-polybenzimidazole electrolyte membrane. Energy Storage Mater. 27, 418-425 (2020). https://doi.org/10.1016/j.ensm.2020.02.016

95. M.M. Abou-Krisha, A.G. Alshammari, F.H. Assaf, F.A. ElSheref, Electrochemical behavior of $\mathrm{Zn}-\mathrm{Co}-\mathrm{Fe}$ alloy electrodeposited from a sulfate bath on various substrate materials. Arab. J. Chem. 12(8), 3526-3533 (2019). https://doi.org/ 10.1016/j.arabjc.2015.10.008

96. Q. Yang, Z. Huang, X. Li, Z. Liu, H. Li et al., A wholly degradable, rechargeable $\mathrm{Zn}-\mathrm{Ti}_{3} \mathrm{C}_{2}$ MXene capacitor with superior anti-self-discharge function. ACS Nano 13(7), 8275-8283 (2019). https://doi.org/10.1021/acsnano.9b03650

97. G. Sun, H. Yang, G. Zhang, J. Gao, X. Jin et al., A capacity recoverable zinc-ion micro-supercapacitor. Energy Environ. Sci. 11(12), 3367-3374 (2018). https://doi.org/10.1039/c8ee0 $2567 \mathrm{c}$

98. L. Lei, Y. Zheng, X. Zhang, Y. Su, X. Zhou et al., A ZIF-8 host for dendrite-free zinc anodes and N, O dual-doped carbon cathodes for high-performance zinc-ion hybrid capacitors. Chem. Asian J. 16(15), 2146-2153 (2021). https://doi. org/10.1002/asia.202100526

99. S. Chen, L. Ma, K. Zhang, M. Kamruzzaman, C. Zhi et al., A flexible solid-state zinc ion hybrid supercapacitor based on co-polymer derived hollow carbon spheres. J. Mater. Chem. A 7(13), 7784-7790 (2019). https://doi.org/10.1039/ c9ta00733d

100. H. Jia, Z. Wang, B. Tawiah, Y. Wang, C. Chan et al., Recent advances in zinc anodes for high-performance aqueous $\mathrm{Zn}$ ion batteries. Nano Energy 70, 104523 (2020). https://doi. org/10.1016/j.nanoen.2020.104523

101. H. Wang, M. Wang, Y. Tang, A novel zinc-ion hybrid supercapacitor for long-life and low-cost energy storage applications. Energy Storage Mater. 13, 1-7 (2018). https:// doi.org/10.1016/j.ensm.2017.12.022

102. C. Leng, Z. Zhao, Y. Song, L. Sun, Z. Fan et al., 3D carbon frameworks for ultrafast charge/discharge rate supercapacitors with high energy-power density. Nano-Micro Lett. 13, 8 (2020). https://doi.org/10.1007/s40820-020-00535-w

103. G. Wang, L. Zhang, J. Zhang, A review of electrode materials for electrochemical supercapacitors. Chem. Soc. Rev. 41(2), 797-828 (2012). https://doi.org/10.1039/c1cs15060j

104. L.G.H. Staaf, P. Lundgren, P. Enoksson, Present and future supercapacitor carbon electrode materials for improved energy storage used in intelligent wireless sensor systems. Nano Energy 9, 128-141 (2014). https://doi.org/10.1016/j. nanoen.2014.06.028

105. Z. Heidarinejad, M.H. Dehghani, M. Heidari, G. Javedan, I. Ali et al., Methods for preparation and activation of activated carbon: a review. Environ. Chem. Lett. 18(2), 393415 (2020). https://doi.org/10.1007/s10311-019-00955-0 
106. A.G. Pandolfo, A.F. Hollenkamp, Carbon properties and their role in supercapacitors. J. Power Sources 157(1), 11-27 (2006). https://doi.org/10.1016/j.jpowsour.2006.02. 065

107. W. Raza, F. Ali, N. Raza, Y. Luo, K.H. Kim et al., Recent advancements in supercapacitor technology. Nano Energy 52, 441-473 (2018). https://doi.org/10.1016/j.nanoen.2018.08. 013

108. J. Yin, W. Zhang, W. Wang, N.A. Alhebshi, N. Salah et al., Electrochemical zinc ion capacitors enhanced by redox reactions of porous carbon cathodes. Adv. Energy Mater. 10(37), 2001705 (2020). https://doi.org/10.1002/aenm.202001705

109. H. Fan, X. Hu, S. Zhang, Z. Xu, G. Gao et al., Flower-like carbon cathode prepared via in situ assembly for $\mathrm{Zn}$-ion hybrid supercapacitors. Carbon 180, 254-264 (2021). https:// doi.org/10.1016/j.carbon.2021.04.093

110. L. Han, H. Huang, J. Li, Z. Yang, X. Zhang et al., Novel zinc-iodine hybrid supercapacitors with a redox iodide ion electrolyte and B, $\mathrm{N}$ dual-doped carbon electrode exhibit boosted energy density. J. Mater. Chem. A 7(42), 2440024407 (2019). https://doi.org/10.1039/c9ta07196b

111. Z. Pan, Z. Lu, L. Xu, D. Wang, A robust 2 D porous carbon nanoflake cathode for high energy-power density $\mathrm{Zn}$ ion hybrid supercapacitor applications. Appl. Surf. Sci. 510, 145384 (2020). https://doi.org/10.1016/j.apsusc.2020.145384

112. H. Fan, S. Zhou, Q. Chen, G. Gao, Q. Ban et al., Phosphorus in honeycomb-like carbon as a cathode boosting pseudocapacitive properties for $\mathrm{Zn}$-ion storage. J. Power Sources $\mathbf{4 9 3}$, 229687 (2021). https://doi.org/10.1016/j.jpowsour.2021. 229687

113. Y. Zhang, Z. Wang, D. Li, Q. Sun, K. Lai et al., Ultrathin carbon nanosheets for highly efficient capacitive K-ion and Zn-ion storage. J. Mater. Chem. A 8(43), 22874-22885 (2020). https://doi.org/10.1039/d0ta08577d

114. S. Zeng, X. Shi, D. Zheng, C. Yao, F. Wang et al., Molten salt assisted synthesis of pitch derived carbon for $\mathrm{Zn}$ ion hybrid supercapacitors. Mater. Res. Bull. 135, 111134 (2021). https://doi.org/10.1016/j.materresbull.2020.111134

115. H. Chen, Y. Zheng, X. Zhu, W. Hong, Y. Tong et al., Bamboo-derived porous carbons for $\mathrm{Zn}$-ion hybrid supercapacitors. Mater. Res. Bull. 139, 111281 (2021). https://doi.org/ 10.1016/j.materresbull.2021.111281

116. C. Hou, Y. Wang, L. Zou, M. Wang, H. Liu et al., A gassteamed MOF route to P-doped open carbon cages with enhanced Zn-ion energy storage capability and ultrastability. Adv. Mater. 33(29), 2101698 (2021). https://doi.org/10. 1002/adma.202101698

117. H. Fan, S. Zhou, Q. Li, G. Gao, Y. Wang et al., Hydrogenbonded frameworks crystals-assisted synthesis of flowerlike carbon materials with penetrable meso/macropores from heavy fraction of bio-oil for $\mathrm{Zn}$-ion hybrid supercapacitors. J. Colloid Interface Sci. 600, 681-690 (2021). https://doi.org/10.1016/j.jcis.2021.05.042

118. L. Wen, F. Li, H. Cheng, Carbon nanotubes and graphene for flexible electrochemical energy storage: from materials to devices. Adv. Mater. 28(22), 4306-4337 (2016). https:// doi.org/10.1002/adma.201504225

119. A. Afzal, F.A. Abuilaiwi, A. Habib, M. Awais, S.B. Waje et al., Polypyrrole/carbon nanotube supercapacitors: technological advances and challenges. J. Power Sources 352, 174-186 (2017). https://doi.org/10.1016/j.jpowsour.2017. 03.128

120. X. Zhang, Z. Pei, C. Wang, Z. Yuan, L. Wei et al., Flexible zinc-ion hybrid fiber capacitors with ultrahigh energy density and long cycling life for wearable electronics. Small 15(47), 1903817 (2019). https://doi.org/10.1002/smll. 201903817

121. Y.H. Tian, R. Amal, D.W. Wang, An aqueous metali-ion capacitor with oxidized carbon nanotubes and metalicn zinc electrodes. Front. Energy Res. 4, 34 (2016). https://doi.org/ 10.3389/fenrg.2016.00034

122. D.S. Hecht, L.B. Hu, G. Irvin, Emerging transparent electrodes based on thin films of carbon nanotubes, graphene, and metallic nanostructures. Adv. Mater. 23(13), 1482-1513 (2011). https://doi.org/10.1002/adma.201003188

123. C. Hu, L. Dai, Doping of carbon materials for metal-free electrocatalysis. Adv. Mater. 31(7), 1804672 (2019). https:// doi.org/10.1002/adma.201804672

124. K. Gao, B. Wang, L. Tao, B.V. Cunning, Z. Zhang et al., Efficient metal-free electrocatalysts from $\mathrm{N}$-doped carbon nanomaterials: mono-doping and Co-doping. Adv. Mater. 31(13), 1805121 (2019). https://doi.org/10.1002/adma.201805121

125. F. Wei, X. He, L. Ma, H. Zhang, N. Xiao et al., 3D N, O-codoped egg-box-like carbons with tuned channels for high areal capacitance supercapacitors. Nano-Micro Lett. 12, 82 (2020). https://doi.org/10.1007/s40820-020-00416-2

126. S. Ghosh, S. Barg, S.M. Jeong, K. Ostrikov, Heteroatomdoped and oxygen-functionalized nanocarbons for highperformance supercapacitors. Adv. Energy Mater. 10(32), 2001239 (2020). https://doi.org/10.1002/aenm.202001239

127. R. Kumar, S. Sahoo, E. Joanni, R.K. Singh, K. Maegawa et al., Heteroatom doped graphene engineering for energy storage and conversion. Mater. Today 39, 47-65 (2020). https://doi.org/10.1016/j.mattod.2020.04.010

128. A. Gopalakrishnan, S. Badhulika, Effect of self-doped heteroatoms on the performance of biomass-derived carbon for supercapacitor applications. J. Power Sources 480, 228830 (2020). https://doi.org/10.1016/j.jpowsour.2020.228830

129. Y. Zheng, H. Song, S. Chen, X. Yu, J. Zhu et al., Metal-free multi-heteroatom-doped carbon bifunctional electrocatalysts derived from a covalent triazine polymer. Small 16(47), 2004342 (2020). https://doi.org/10.1002/smll.202004342

130. P. Liu, Y. Gao, Y. Tan, W. Liu, Y. Huang et al., Rational design of nitrogen doped hierarchical porous carbon for optimized zinc-ion hybrid supercapacitors. Nano Res. 12(11), 2835-2841 (2019). https://doi.org/10.1007/ s12274-019-2521-6

131. J. Han, K. Wang, W. Liu, C. Li, X. Sun et al., Rational design of nano-architecture composite hydrogel electrode towards high performance Zn-ion hybrid cell. Nanoscale 10(27), 13083-13091 (2018). https://doi.org/10.1039/c8nr03889a 
132. X. Deng, J. Li, Z. Shan, J. Sha, L. Ma et al., A N, O co-doped hierarchical carbon cathode for high-performance $\mathrm{Zn}$-ion hybrid supercapacitors with enhanced pseudocapacitance. J. Mater. Chem. A 8(23), 11617-11625 (2020). https://doi.org/ 10.1039/d0ta02770g

133. Y. Lee, G. An, Synergistic effects of phosphorus and boron Co-incorporated activated carbon for ultrafast zinc-ion hybrid supercapacitors. ACS Appl. Mater. Interfaces 12(37), 4134241349 (2020). https://doi.org/10.1021/acsami.0c10512

134. Y. Zhu, S. Murali, M.D. Stoller, K.J. Ganesh, W. Cai et al., Carbon-based supercapacitors produced by activation of graphene. Science 332(6037), 1537-1541 (2011). https://doi.org/ 10.1126/science. 1200770

135. Y. Zhu, S. Murali, W. Cai, X. Li, J.W. Suk et al., Graphene and graphene oxide: synthesis, properties, and applications. Adv. Mater. 22(35), 3906-3924 (2010). https://doi.org/10. 1002/adma.201001068

136. J. Du, Q. Cao, X. Tang, X. Xu, X. Long et al., 3D printingassisted gyroidal graphite foam for advanced supercapacitors. Chem. Eng. J. 416, 127885 (2021). https://doi.org/10.1016/j. cej.2020.127885

137. J. Xia, F. Chen, J. Li, N. Tao, Measurement of the quantum capacitance of graphene. Nat. Nanotechnol. 4(8), 505-509 (2009). https://doi.org/10.1038/nnano.2009.177

138. J.R. Miller, R.A. Outlaw, B.C. Holloway, Graphene doublelayer capacitor with ac line-filtering performance. Science 329(5999), 1637-1639 (2010). https://doi.org/10.1126/scien ce. 1194372

139. Q. Wang, S. Wang, X. Guo, L. Ruan, N. Wei et al., MXenereduced graphene oxide aerogel for aqueous zinc-ion hybrid supercapacitor with ultralong cycle life. Adv. Electron. Mater. 5(12), 1900537 (2019). https://doi.org/10.1002/aelm.20190 0537

140. Y. Xu, X. Chen, C. Huang, Y. Zhou, B. Fan et al., Redoxactive p-phenylenediamine functionalized reduced graphene oxide film through covalently grafting for ultrahigh areal capacitance Zn-ion hybrid supercapacitor. J. Power Sources 488, 229426 (2021). https://doi.org/10.1016/j.jpowsour.2020. 229426

141. P. Simon, Y. Gogotsi, Perspectives for electrochemical capacitors and related devices. Nat. Mater. 19(11), 1151-1163 (2020). https://doi.org/10.1038/s41563-020-0747-z

142. N.R. Chodankar, H.D. Pham, A.K. Nanjundan, J.F.S. Fernando, K. Jayaramulu et al., True meaning of pseudocapacitors and their performance metrics: asymmetric versus hybrid supercapacitors. Small 16(37), 2002806 (2020). https://doi. org/10.1002/smll.202002806

143. S. Kumar, G. Saeed, L. Zhu, K.N. Hui, N.H. Kim et al., OD to 3D carbon-based networks combined with pseudocapacitive electrode material for high energy density supercapacitor: a review. Chem. Eng. J. 403, 126352 (2021). https://doi.org/10. 1016/j.cej.2020.126352

144. P. Das, Z.S. Wu, MXene for energy storage: present status and future perspectives. J. Phys-Energy 2(3), 032004 (2020). https://doi.org/10.1088/2515-7655/ab9b1d
145. C.E. Ren, M. Zhao, T. Makaryan, J. Halim, M. Boota et al., Porous two-dimensional transition metal carbide (MXene) flakes for high-performance Li-ion storage. ChemElectroChem 3(5), 689-693 (2016). https://doi.org/10.1002/celc. 201600059

146. Y. Xie, M. Naguib, V.N. Mochalin, M.W. Barsoum, Y. Gogotsi et al., Role of surface structure on Li-ion energy storage capacity of two-dimensional transition-metal carbides. J. Am. Chem. Soc. 136(17), 6385-6394 (2014). https://doi.org/ 10.1021/ja501520b

147. M.A.M. Hasan, Y. Wang, C.R. Bowen, Y. Yang, 2D nanomaterials for effective energy scavenging. Nano-Micro Lett. 13, 82 (2021). https://doi.org/10.1007/s40820-021-00603-9

148. M.R. Lukatskaya, O. Mashtalir, C.E. Ren, Y. Dall'Agnese, P. Rozier et al., Cation intercalation and high volumetric capacitance of two-dimensional titanium carbide. Science 341(6153), 1502-1505 (2013). https://doi.org/10.1126/scien ce. 1241488

149. P.C. Ruan, X.L. Xu, J.X. Feng, L.H. Yu, X.L. Gao et al., Boosting zinc storage performance via conductive materials. Mater. Res. Bull. 133, 111077 (2021). https://doi.org/10. 1016/j.materresbull.2020.111077

150. J. Come, J.M. Black, M.R. Lukatskaya, M. Naguib, M. Beidaghi et al., Controlling the actuation properties of MXene paper electrodes upon cation intercalation. Nano Energy 17, 27-35 (2015). https://doi.org/10.1016/j.nanoen.2015.07.028

151. B. Anasori, M.R. Lukatskaya, Y. Gogotsi, 2D metal carbides and nitrides (MXenes) for energy storage. Nat. Rev. Mater. 2(2), 16098 (2017). https://doi.org/10.1038/natrevmats.2016. 98

152. M. Ghidiu, M.R. Lukatskaya, M. Zhao, Y. Gogotsi, M.W. Barsoum, Conductive two-dimensional titanium carbide "clay" with high volumetric capacitance. Nature 516(7529), 78-81 (2014). https://doi.org/10.1038/nature13970

153. L. Li, W. Liu, K. Jiang, D. Chen, F. Qu et al., In-situ annealed $\mathrm{Ti}_{3} \mathrm{C}_{2} \mathrm{~T}_{\mathrm{x}}$ MXene based all-solid-state flexible $\mathrm{Zn}$ ion hybrid micro supercapacitor array with enhanced stability. Nano-Micro Lett. 13, 100 (2021). https://doi.org/10.1007/ s40820-021-00634-2

154. C. Huang, X. Zhao, Y. Xu, Y. Zhang, Y. Yang et al., Sewable and cuttable flexible zinc-ion hybrid supercapacitor using a polydopamine/carbon cloth-based cathode. ACS Sustain. Chem. Eng. 8(42), 16028-16036 (2020). https://doi.org/10. 1021/acssuschemeng.0c06525

155. Y. Li, W. Yang, W. Yang, Z. Wang, J. Rong et al., Towards high-energy and anti-self-discharge $\mathrm{Zn}$-ion hybrid supercapacitors with new understanding of the electrochemistry. Nano-Micro Lett. 13, 95 (2021). https://doi.org/10.1007/ s40820-021-00625-3

156. K. Fic, G. Lota, E. Frackowiak, Effect of surfactants on capacitance properties of carbon electrodes. Electrochim. Acta 60, 206-212 (2012). https://doi.org/10.1016/j.electacta. 2011.11.059

157. F. Mo, G. Liang, Q. Meng, Z. Liu, H. Li et al., A flexible rechargeable aqueous zinc manganese-dioxide battery 
working at $-20{ }^{\circ} \mathrm{C}$. Energy Environ. Sci. 12(2), 706-715 (2019). https://doi.org/10.1039/c8ee02892c

158. J. Lee, A. Tolosa, B. Kruener, N. Jaeckel, S. Fleischmann et al., Asymmetric tin-vanadium redox electrolyte for hybrid energy storage with nanoporous carbon electrodes. Sustain. Energ. Fuels 1(2), 299-307 (2017). https://doi.org/10.1039/ c6se00062b

159. J. Wang, B. Ding, X. Hao, Y. Xu, Y. Wang et al., A modified molten-salt method to prepare graphene electrode with high capacitance and low self-discharge rate. Carbon 102, 255-261 (2016). https://doi.org/10.1016/j.carbon.2016.02. 047

160. K. Wang, H. Wu, Y. Meng, Z. Wei, Conducting polymer nanowire arrays for high performance supercapacitors. Small 10(1), 14-31 (2014). https://doi.org/10.1002/smll.201301991

161. V. Augustyn, P. Simon, B. Dunn, Pseudocapacitive oxide materials for high-rate electrochemical energy storage. Energy Environ. Sci. 7(5), 1597-1614 (2014). https://doi. org/10.1039/C3EE44164D

162. K. Ren, Z. Liu, T. Wei, Z. Fan, Recent developments of transition metal compounds-carbon hybrid electrodes for high energy/power supercapacitors. Nano-Micro Lett. 13, 129 (2021). https://doi.org/10.1007/s40820-021-00642-2

163. Q. Jiang, N. Kurra, M. Alhabeb, Y. Gogotsi, H.N. Alshareef,

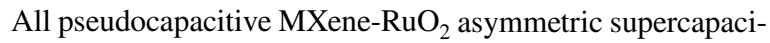
tors. Adv. Energy Mater. 8(13), 1703043 (2018). https://doi. org/10.1002/aenm.201703043

164. V. Augustyn, J. Come, M.A. Lowe, J.W. Kim, P.L. Taberna et al., High-rate electrochemical energy storage through $\mathrm{Li}^{+}$ intercalation pseudocapacitance. Nat. Mater. 12(6), 518-522 (2013). https://doi.org/10.1038/nmat3601

165. L. Dong, W. Yang, W. Yang, C. Wang, Y. Li et al., Highpower and ultralong-life aqueous zinc-ion hybrid capacitors based on pseudocapacitive charge storage. Nano-Micro Lett. 11, 94 (2019). https://doi.org/10.1007/s40820-019-0328-3

166. P. Yang, D. Chao, C. Zhu, X. Xia, Y. Zhang et al., Ultrafastcharging supercapacitors based on corn-like titanium nitride nanostructures. Adv. Sci. 3(6), 1500299 (2016). https://doi. org/10.1002/advs.201500299

167. X. Lu, G. Wang, T. Zhai, M. Yu, S. Xie et al., Stabilized TiN nanowire arrays for high-performance and flexible supercapacitors. Nano Lett. 12(10), 5376-5381 (2012). https://doi. org/10.1021/nl302761z

168. B. Yao, M. Li, J. Zhang, L. Zhang, Y. Song et al., TiN paper for ultrafast-charging supercapacitors. Nano-Micro Lett. 12, 3 (2019). https://doi.org/10.1007/s40820-019-0340-7

169. Z.D. Huang, T.R. Wang, H. Song, X.L. Li, G.J. Liang et al., Effects of anion carriers on capacitance and self-discharge behaviors of zinc ion capacitors. Angew. Chem. Int. Ed. 60(2), 1011-1021 (2021). https://doi.org/10.1002/anie.20201 2202

170. C. Wang, Z. Pei, Q. Meng, C. Zhang, X. Sui et al., Toward flexible zinc-ion hybrid capacitors with superhigh energy density and ultralong cycling life: the pivotal role of $\mathrm{ZnCl}_{2}$ salt-based electrolytes. Angew. Chem. Int. Ed. 60(2), 990997 (2021). https://doi.org/10.1002/anie.202012030
171. D. Wang, Z. Pan, Z. Lu, From starch to porous carbon nanosheets: promising cathodes for high-performance aqueous Zn-ion hybrid supercapacitors. Microporous Mesoporous Mater. 306, 110445 (2020). https://doi.org/10.1016/j.micro meso.2020.110445

172. X. Tuo, W. Yinong, W. Na, Z. Yi, L. Huan et al., A highcapacity aqueous $\mathrm{Zn}$-ion hybrid energy storage device using poly (4,4'-thiodiphenol) modified activated carbon as a cathode material. J. Mater. Chem. A 7(40), 23076-23083 (2019). https://doi.org/10.1039/c9ta08693e

173. K. Zhang, X. Han, Z. Hu, X. Zhang, Z. Tao et al., Nanostructured Mn-based oxides for electrochemical energy storage and conversion. Chem. Soc. Rev. 44(3), 699-728 (2015). https://doi.org/10.1039/c4cs00218k

174. N. Zhang, Y. Dong, M. Jia, X. Bian, Y. Wang et al., Rechargeable aqueous $\mathrm{Zn}-\mathrm{V}_{2} \mathrm{O}_{5}$ battery with high energy density and long cycle life. ACS Energy Lett. 3(6), 1366-1372 (2018). https://doi.org/10.1021/acsenergylett.8b00565

175. J.S. Park, J.H. Jo, Y. Aniskevich, A. Bakavets, G. Ragoisha et al., Open-structured vanadium dioxide as an intercalation host for $\mathrm{Zn}$ ions: investigation by first-principles calculation and experiments. Chem. Mater. 30(19), 6777-6787 (2018). https://doi.org/10.1021/acs.chemmater.8b02679

176. P. He, G. Zhang, X. Liao, M. Yan, X. Xu et al., Sodium ion stabilized vanadium oxide nanowire cathode for high-performance zinc-ion batteries. Adv. Energy Mater. 8(10), 1702463 (2018). https://doi.org/10.1002/aenm.201702463

177. P. He, Y. Quan, X. Xu, M. Yan, W. Yang et al., High-performance aqueous zinc-ion battery based on layered $\mathrm{H}_{2} \mathrm{~V}_{3} \mathrm{O}_{8}$ nanowire cathode. Small 13(47), 1702551 (2017). https://doi. org/10.1002/smll.201702551

178. Q. Liu, Z. Hu, M. Chen, C. Zou, H. Jin et al., The cathode choice for commercialization of sodium-ion batteries: layered transition metal oxides versus prussian blue analogs. Adv. Funct. Mater. 30(14), 1909530 (2020). https://doi.org/ 10.1002/adfm.201909530

179. G. Zampardi, F.L. Mantia, Prussian blue analogues as aqueous $\mathrm{Zn}$-ion batteries electrodes: current challenges and future perspectives. Curr. Opin. Electroche. 21, 84-92 (2020). https://doi.org/10.1016/j.coelec.2020.01.014

180. G. Du, H. Pang, Recent advancements in prussian blue analogues: preparation and application in batteries. Energy Storage Mater. 36, 387-408 (2021). https://doi.org/10. 1016/j.ensm.2021.01.006

181. H. Zhang, J. Wang, Q. Liu, W. He, Z. Lai et al., Extracting oxygen anions from $\mathrm{ZnMn}_{2} \mathrm{O}_{4}$ : robust cathode for flexible all-solid-state Zn-ion batteries. Energy Storage Mater. 21, 154-161 (2019). https://doi.org/10.1016/j.ensm.2018.12. 019

182. S. Peng, L. Li, Y. Hu, M. Srinivasan, F. Cheng et al., Fabrication of spinel one-dimensional architectures by singlespinneret electrospinning for energy storage applications. ACS Nano 9(2), 1945-1954 (2015). https://doi.org/10.1021/ nn506851x

183. W. Li, K. Wang, S. Cheng, K. Jiang, An ultrastable presodiated titanium disulfide anode for aqueous "rocking-chair" 
zinc ion battery. Adv. Energy Mater. 9(27), 1900993 (2019). https://doi.org/10.1002/aenm.201900993

184. Y. Deng, L. Wan, Y. Xie, X. Qin, G. Chen, Recent advances in Mn-based oxides as anode materials for lithium ion batteries. RSC Adv. 4(45), 23914-23935 (2014). https://doi.org/ $10.1039 / \mathrm{c} 4 \mathrm{ra02686a}$

185. L. Chen, C. Hao, Y. Zhang, Y. Wei, L. Dai et al., Guest ions pre-intercalation strategy of manganese-oxides for supercapacitor and battery applications. J. Energy Chem. 60, 480493 (2021). https://doi.org/10.1016/j.jechem.2021.01.023

186. Y. Li, D. Zhang, S. Huang, H.Y. Yang, Guest-species-incorporation in manganese/vanadium-based oxides: Towards high performance aqueous zinc-ion batteries. Nano Energy 85, 105969 (2021). https://doi.org/10.1016/j.nanoen.2021. 105969

187. X. Wang, Z. Zhou, Z. Sun, J. Hah, Y. Yao et al., Atomic modulation of 3D conductive frameworks boost performance of $\mathrm{MnO}_{2}$ for coaxial fiber-shaped supercapacitors. Nano-Micro Lett. 13, 4 (2020). https://doi.org/10.1007/ s40820-020-00529-8

188. X. Ma, J. Cheng, L. Dong, W. Liu, J. Mou et al., Multivalent ion storage towards high-performance aqueous zinc-ion hybrid supercapacitors. Energy Storage Mater. 20, 335-342 (2019). https://doi.org/10.1016/j.ensm.2018.10.020

189. X. Ma, J. Wang, X. Wang, L. Zhao, C. Xu, Aqueous $\mathrm{V}_{2} \mathrm{O}_{5} /$ activated carbon zinc-ion hybrid capacitors with high energy density and excellent cycling stability. J. Mater. Sci.-Mater. Electron. 30(6), 5478-5486 (2019). https://doi.org/10.1007/ s10854-019-00841-z

190. S. Wang, Q. Wang, W. Zeng, M. Wang, L. Ruan et al., A new free-standing aqueous zinc-ion capacitor based on $\mathrm{MnO}_{2}-$ CNTs cathode and MXene anode. Nano-Micro Lett. 11(1), 70 (2019). https://doi.org/10.1007/s40820-019-0301-1

191. S. Zuo, X. Xu, S. Ji, Z. Wang, Z. Liu et al., Cathodes for aqueous $\mathrm{Zn}$-ion batteries: materials, mechanisms, and kinetics. Chem. Eur. J. 27(3), 830-860 (2021). https://doi.org/10. 1002/chem.202002202

192. D. Selvakumaran, A. Pan, S. Liang, G. Cao, A review on recent developments and challenges of cathode materials for rechargeable aqueous $\mathrm{Zn}$-ion batteries. J. Mater. Chem. A 7(31), 18209-18236 (2019). https://doi.org/10.1039/c9ta0 $5053 \mathrm{a}$

193. X. Gong, J. Chen, P.S. Lee, Zinc-ion hybrid supercapacitors: progress and future perspective. Batteries Supercaps 4(10), 1529-1546 (2021). https://doi.org/10.1002/batt.202100034

194. M. Li, Z. Li, X. Wang, J. Meng, X. Liu et al., Comprehensive understanding of the roles of water molecules in aqueous $\mathrm{Zn}$ ion batteries: from electrolytes to electrode materials. Energy Environ. Sci. 14(7), 3796-3839 (2021). https://doi.org/10. 1039/d1ee00030f

195. L. Yuan, J. Hao, C.C. Kao, C. Wu, H.K. Liu et al., Regulation methods for the Zn/electrolyte interphase and the effectiveness evaluation in aqueous Zn-ion batteries. Energy Environ. Sci. 14(11), 5669-5689 (2021). https://doi.org/10.1039/d1ee0 $2021 \mathrm{~h}$
196. X. Ma, J. Cheng, L. Dong, W. Liu, J. Mou et al., Multivalent ion storage towards high-performance aqueous zinc-ion hybrid supercapacitors. Energy Storage Mater. 20, 335-342 (2018). https://doi.org/10.1016/j.ensm.2018.10.020

197. Z. Li, D. Chen, Y. An, C. Chen, L. Wu et al., Flexible and anti-freezing quasi-solid-state zinc ion hybrid supercapacitors based on pencil shavings derived porous carbon. Energy Storage Mater. 28, 307-314 (2020). https://doi.org/10.1016/j. ensm.2020.01.028

198. L. Han, H. Huang, J. Li, X. Zhang, Z. Yang et al., A novel redox bromide-ion additive hydrogel electrolyte for flexible Zn-ion hybrid supercapacitors with boosted energy density and controllable zinc deposition. J. Mater. Chem. A 8(30), 15042-15050 (2020). https://doi.org/10.1039/d0ta03547e

199. N. Chang, T. Li, R. Li, S. Wang, Y. Yin et al., An aqueous hybrid electrolyte for low-temperature zinc-based energy storage devices. Energy Environ. Sci. 13(10), 3527-3535 (2020). https://doi.org/10.1039/d0ee01538e

200. F. Li, L. Yu, Q. Hu, S. Guo, Y. Mei et al., Fabricating lowtemperature-tolerant and durable $\mathrm{Zn}$-ion capacitors via modulation of co-solvent molecular interaction and cation solvation. Sci. China-Mater. 64(7), 1609-1620 (2021). https://doi. org/10.1007/s40843-020-1570-5

201. H. Zhang, E.G. Gebresilassie, X. Judez, C. Li, M.L.M. Rodriguez et al., Electrolyte additives for lithium metal anodes and rechargeable lithium metal batteries: progress and perspectives. Angew. Chem. Int. Ed. 57(46), 15002-15027 (2018). https://doi.org/10.1002/anie.201712702

202. A.M. Haregewoin, A.S. Wotango, B.J. Hwang, Electrolyte additives for lithium ion battery electrodes: progress and perspectives. Energy Environ. Sci. 9(6), 1955-1988 (2016). https://doi.org/10.1039/c6ee00123h

203. M. Li, Q. He, Z. Li, Q. Li, Y. Zhang et al., A novel dendritefree $\mathrm{Mn}^{2+} / \mathrm{Zn}^{2+}$ hybrid battery with $2.3 \mathrm{~V}$ voltage window and 11000-cycle lifespan. Adv. Energy Mater. 9(29), 1901469 (2019). https://doi.org/10.1002/aenm.201901469

204. S. Zhao, B. Han, D. Zhang, Q. Huang, L. Xiao et al., Unravelling the reaction chemistry and degradation mechanism in aqueous $\mathrm{Zn} / \mathrm{MnO}_{2}$ rechargeable batteries. J. Mater. Chem. A 6(14), 5733-5739 (2018). https://doi.org/10.1039/c8ta01031e

205. D. Gong, B. Wang, J. Zhu, R. Podila, A.M. Rao et al., An iodine quantum dots based rechargeable sodium-iodine battery. Adv. Energy Mater. 7(3), 1601885 (2017). https://doi. org/10.1002/aenm.201601885

206. K. Lu, H. Zhang, F. Ye, W. Luo, H. Ma et al., Rechargeable potassium-ion batteries enabled by potassium-iodine conversion chemistry. Energy Storage Mater. 16, 1-5 (2019). https:// doi.org/10.1016/j.ensm.2018.04.018

207. Z. Zhao, J. Zhao, Z. Hu, J.D. Li, J.J. Li et al., Long-life and deeply rechargeable aqueous $\mathrm{Zn}$ anodes enabled by a multifunctional brightener-inspired interphase. Energy Environ. Sci. 12(6), 1938-1949 (2019). https://doi.org/10.1039/c9ee0 $0596 \mathrm{j}$

208. Y. Tang, C. Liu, H. Zhu, X. Xie, J. Gao et al., Ion-confinement effect enabled by gel electrolyte for highly reversible 
dendrite-free zinc metal anode. Energy Storage Mater. 27, 109-116 (2020). https://doi.org/10.1016/j.ensm.2020.01.023

209. P. Zhang, F. Wang, S. Yang, G. Wang, M. Yu et al., Flexible in-plane micro-supercapacitors: progresses and challenges in fabrication and applications. Energy Storage Mater. 28, 160-187 (2020). https://doi.org/10.1016/j.ensm.2020.02.029

210. F. Bu, W. Zhou, Y. Xu, Y. Du, C. Guan et al., Recent developments of advanced micro-supercapacitors: design, fabrication and applications. npj Flex. Electron. 4(1), 31 (2020). https:// doi.org/10.1038/s41528-020-00093-6

211. B.D. Boruah, S. Nandi, A. Misra, Layered assembly of reduced graphene oxide and vanadium oxide heterostructure supercapacitor electrodes with larger surface area for efficient energy-storage performance. ACS Appl. Energy Mater. 1(4), 1567-1574 (2018). https://doi.org/10.1021/acsaem.7b00358

212. W. Liu, Y. Feng, X. Yan, J. Chen, Q. Xue, Superior microsupercapacitors based on graphene quantum dots. Adv. Funct. Mater. 23(33), 4111-4122 (2013). https://doi.org/10.1002/ adfm.201203771

213. L. Li, J. Zhang, Z. Peng, Y. Li, C. Gao et al., High-performance pseudocapacitive microsupercapacitors from laserinduced graphene. Adv. Mater. 28(5), 838-845 (2016). https://doi.org/10.1002/adma.201503333

214. K. Robert, C. Douard, A. Demortiere, F. Blanchard, P. Roussel et al., On chip interdigitated micro-supercapacitors based on sputtered bifunctional vanadium nitride thin films with finely tuned inter- and intracolumnar porosities. Adv. Mater. Technol. 3(7), 1800036 (2018). https://doi.org/10.1002/admt.201800036

215. K. Shen, J. Ding, S. Yang, 3D printing quasi-solid-state asymmetric micro-supercapacitors with ultrahigh areal energy density. Adv. Energy Mater. 8(20), 1800408 (2018). https://doi. org/10.1002/aenm.201800408

216. W. Liu, X. Yan, J. Chen, Y. Feng, Q. Xue, Novel and highperformance asymmetric micro-supercapacitors based on graphene quantum dots and polyaniline nanofibers. Nanoscale 5(13), 6053-6062 (2013). https://doi.org/10. 1039/c3nr01139a

217. Y. Peng, B. Akuzum, N. Kurra, M. Zhao, M. Alhabe et al., All-MXene (2D titanium carbide) solid-state microsupercapacitors for on-chip energy storage. Energy Environ. Sci. 9(9), 2847-2854 (2016). https://doi.org/10.1039/c6ee01717g

218. S.C. Lee, U.M. Patil, S.J. Kim, S. Ahn, S. Kang et al., Allsolid-state flexible asymmetric micro supercapacitors based on cobalt hydroxide and reduced graphene oxide electrodes. RSC Adv. 6(50), 43844-43854 (2016). https://doi.org/10. 1039/c6ra06034j

219. H. Hu, Z. Pei, H. Fan, C. Ye, $3 \mathrm{D}$ interdigital $\mathrm{Au} / \mathrm{MnO}_{2} / \mathrm{Au}$ stacked hybrid electrodes for on-chip microsupercapacitors. Small 12(22), 3059-3069 (2016). https://doi.org/10.1002/ smll.201503527

220. X. Wang, X. Lu, B. Liu, D. Chen, Y. Tong et al., Flexible energy-storage devices: design consideration and recent progress. Adv. Mater. 26(28), 4763-4782 (2014). https://doi.org/ 10.1002/adma.201400910
221. L. Li, Z. Wu, S. Yuan, X. Zhang, Advances and challenges for flexible energy storage and conversion devices and systems. Energy Environ. Sci. 7(7), 2101-2122 (2014). https://doi.org/ 10.1039/c4ee00318g

222. Y. Yan, X. Liu, J. Yan, C. Guan, J. Wang, Electrospun nanofibers for new generation flexible energy storage. Energy Environ. Mater. 4(4), 502-521 (2021). https://doi.org/10. 1002/eem2.12146

223. K.N. Kang, A. Ramadoss, J.W. Min, J.C. Yoon, D. Lee et al., Wire-shaped 3D-hybrid supercapacitors as substitutes for batteries. Nano-Micro Lett. 12, 28 (2020). https://doi.org/10. 1007/s40820-019-0356-z

224. H.B. Dong, J.W. Li, J. Guo, F.L. Lai, F.J. Zhao et al., Insights on flexible zinc-ion batteries from lab research to commercialization. Adv. Mater. 33(20), 2007548 (2021). https://doi. org/10.1002/adma.202007548

225. F. Wan, L. Zhang, X. Wang, S. Bi, Z. Niu et al., An aqueous rechargeable zinc-organic battery with hybrid mechanism. Adv. Funct. Mater. 28(45), 1804975 (2018). https://doi.org/ 10.1002/adfm.201804975

226. F. Wan, L. Zhang, X. Dai, X. Wang, Z. Niu et al., Aqueous rechargeable zinc/sodium vanadate batteries with enhanced performance from simultaneous insertion of dual carriers. Nat. Commun. 9, 1656 (2018). https://doi.org/10.1038/ s41467-018-04060-8

227. Z. Wang, Z. Ruan, W.S. Ng, H. Li, Z. Tang et al., Integrating a triboelectric nanogenerator and a zinc-ion battery on a designed flexible 3D spacer fabric. Small Methods 2(10), 1800150 (2018). https://doi.org/10.1002/smtd.201800150

228. L.V. Thong, H. Kim, A. Ghosh, J. Kim, J. Chang et al., Coaxial fiber supercapacitor using all-carbon material electrodes. ACS Nano 7(7), 5940-5947 (2013). https://doi.org/10.1021/ $\mathrm{nn} 4016345$

229. Y. Huang, H. Hu, Y. Huang, M. Zhu, W. Meng et al., From industrially weavable and knittable highly conductive yarns to large wearable energy storage textiles. ACS Nano 9(5), 4766-4775 (2015). https://doi.org/10.1021/acsnano.5b00860

230. K. Guo, Y. Ma, H. Li, T. Zhai, Flexible wire-shaped supercapacitors in parallel double helix configuration with stable electrochemical properties under static/dynamic bending. Small 12(8), 1024-1033 (2016). https://doi.org/10.1002/smll. 201503021

231. J. Ren, W. Bai, G. Guan, Y. Zhang, H. Peng, Flexible and weaveable capacitor wire based on a carbon nanocomposite fiber. Adv. Mater. 25(41), 5965-5970 (2013). https://doi.org/ 10.1002/adma.201302498

232. X. Pu, L. Li, M. Liu, C. Jiang, C. Du et al., Wearable selfcharging power textile based on flexible yarn supercapacitors and fabric nanogenerators. Adv. Mater. 28(1), 98-105 (2016). https://doi.org/10.1002/adma.201504403

233. P. Xu, T. Gu, Z. Cao, B. Wei, J. Yu et al., Carbon nanotube fiber based stretchable wire-shaped supercapacitors. Adv. Energy Mater. 4(3), 1300759 (2014). https://doi.org/10.1002/ aenm.201300759 


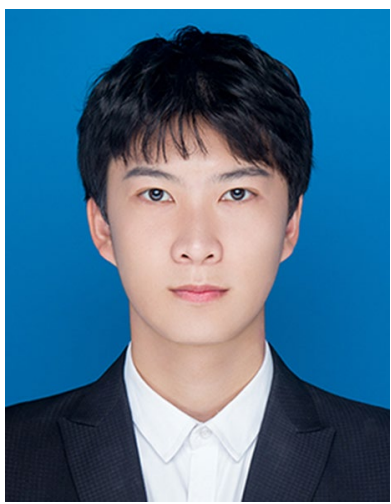

Jialun Jin received his Bachelor's degree from Wuhan University of Technology, Wuhan, China in 2021. He is currently a M.S. degree candidate in School of Integrated Circuits from Tsinghua University, Beijing, China. His current research focuses on Zn-ion hybrid supercapacitors and photoelectronic devices.

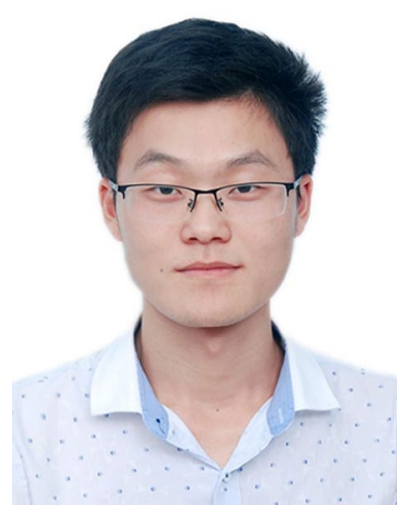

Xiangshun Geng received his $\mathrm{Ph} . \mathrm{D}$. degree in electronic science and technology from Tsinghua University, Beijing, China, in 2021 . He is currently a postdoctoral fellow in Tsinghua University. His current research area concentrates on novel materials and photoelectronic devices.

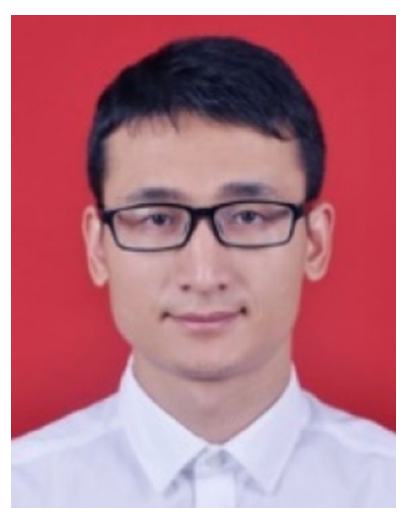

Qiang Chen received his Ph.D. degree from Wuhan University of Technology in 2021. Dr. Chen as joint training doctoral student at National University of Singapore in 2019-2020. He is currently an associate professor of Materials Science and Engineering at College of Materials Science and Engineering, Zhejiang University of Technology, China. His current research involves nanomaterials and devices for energy storage.

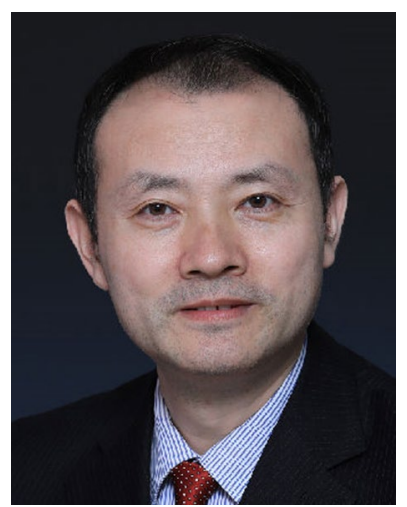

Tian-Ling Ren received his $\mathrm{Ph} . \mathrm{D}$. degree in solid-state physics from the Department of Modern Applied Physics, Tsinghua University, Beijing, China, in 1997, where he has been a Full Professor with the Institute of Microelectronics since 2003. His main research interests include 2D material-based devices and novel nano-electronic devices, intelligent sensors and integrated micro-electromechanical systems, and critical technology for advanced micro- and nanoelectronics. 